\title{
Absolute dimensions of eclipsing binaries. XXVI.
}

\section{Setting a new standard: Masses, radii, and abundances for the F-type systems AD Bootis, VZ Hydrae, and WZ Ophiuchi $\star, \star \star$}

\author{
J. V. Clausen ${ }^{1}$, G. Torres ${ }^{2}$, H. Bruntt ${ }^{1,3}$, J. Andersen ${ }^{1,4}$, B. Nordström ${ }^{1}$, R. P. Stefanik ${ }^{2}$, \\ D. W. Latham ${ }^{2}$, and J. Southworth ${ }^{1,5}$
}

${ }^{1}$ Niels Bohr Institute, Copenhagen University, Juliane Maries Vej 30, 2100 Copenhagen Ø, Denmark e-mail: jvc@astro.ku.dk

2 Harvard-Smithsonian Center for Astrophysics, 60 Garden Street, Cambridge, MA 02138, USA

School of Physics A28, University of Sydney, 2006 NSW, Australia

4 Nordic Optical Telescope Scientific Association, Apartado 474, 38700 Santa Cruz de La Palma, Spain

5 Department of Physics, University of Warwick, Coventry CV47AL, UK

Received 28 February 2008 / Accepted 5 April 2008

ABSTRACT

\begin{abstract}
Context. Accurate mass, radius, and abundance determinations from binaries provide important information on stellar evolution, fundamental to central fields in modern astrophysics and cosmology.

Aims. We aim to determine absolute dimensions and abundances for the three F-type main-sequence detached eclipsing binaries AD Boo, VZ Hya, and WZ Oph and to perform a detailed comparison with results from recent stellar evolutionary models.

Methods. uvby light curves and uvby $\beta$ standard photometry were obtained with the Strömgren Automatic Telescope at ESO, La Silla, radial velocity observations at CfA facilities, and supplementary high-resolution spectra with ESO's FEROS spectrograph. State-ofthe-art methods were applied for the analyses: the EBOP and Wilson-Devinney binary models, two-dimensional cross-correlation and disentangling, and the VWA abundance analysis tool.

Results. Masses and radii that are precise to $0.5-0.7 \%$ and $0.4-0.9 \%$, respectively, have been established for the components, which span the ranges of 1.1 to $1.4 M_{\odot}$ and 1.1 to $1.6 R_{\odot}$. The $[\mathrm{Fe} / \mathrm{H}]$ abundances are from -0.27 to +0.10 , with uncertainties between 0.07 and 0.15 dex. We find indications of a slight $\alpha$-element overabundance of $[\alpha / \mathrm{Fe}] \sim+0.1$ for WZ Oph. The secondary component of $\mathrm{AD}$ Boo and both components of WZ Oph appear to be slightly active. Yale-Yonsai and Victoria-Regina evolutionary models fit the components of AD Boo and VZ Hya almost equally well, assuming coeval formation, at ages of about 1.75/1.50 Gyr (AD Boo) and 1.25/1.00 Gyr (VZ Hya). BaSTI models, however, predict somewhat different ages for the primary and secondary components. For WZOph, the models from all three grids are significantly hotter than observed. A low He content, decreased envelope convection coupled with surface activity, and/or higher interstellar absorption would remove the discrepancy, but its cause has not been definitively identified.

Conclusions. We have demonstrated the power of testing and comparing recent stellar evolutionary models using eclipsing binaries, provided their abundances are known. The strongest limitations and challenges are set by $T_{\text {eff }}$ and interstellar absorption determinations, and by their effects on and correlation with abundance results.
\end{abstract}

Key words. stars: evolution - stars: fundamental parameters - stars: binaries: close - stars: binaries: eclipsing techniques: photometric - techniques: spectroscopic

\section{Introduction}

Accurate stellar mass and radius data from eclipsing binary systems are valuable empirical test data for stellar evolution models, basically because they are free of any scale dependent calibrations. Additional tests become possible when the effective

\footnotetext{
* Based on observations carried out at the Strömgren Automatic Telescope (SAT) and the $1.5 \mathrm{~m}$ and $2.2 \mathrm{~m}$ telescopes at ESO, La Silla, Chile (62.L-0284, 63.H-0080, 71.D-0554); the $1.5 \mathrm{~m}$ Wyeth reflector at the Oak Ridge Observatory, Harvard, Massachusetts, USA; the 1.5-m Tillinghast reflector and the Multiple Mirror Telescope at the F. L. Whipple Observatory, Mt. Hopkins, Arizona.

$\star \star$ Tables A.1-A.3 are available in electronic form at

http: //www . aanda. org and also at the CDS via anonymous ftp to cdsarc.u-strasbg.fr (130.79.128.5) or via

http://cdsweb.u-strasbg.fr/cgi-bin/qcat? J/A+A/487/1095
}

temperatures for the components can also be determined, most often from well-calibrated photometry. The binaries then also serve as primary distance indicators, e.g. for nearby stars, stellar clusters, and Local Group galaxies, and they define the empirical mass-luminosity relations over a broad mass range. However, the most stringent model tests require that spectroscopic element abundances are available as well, minimising the number of free parameters in the comparison with theory (e.g. Andersen 1991). Unfortunately, the number of binary components with complete and accurate data is still small.

In our efforts to provide such complete data for a larger sample of eclipsing binary systems, F-G type main-sequence stars have been given high priority for two reasons. First, their spectra offer favourable conditions for accurate radial velocity determinations and abundance analyses. Second, such stars cover the mass range within which convective cores begin to develop and 


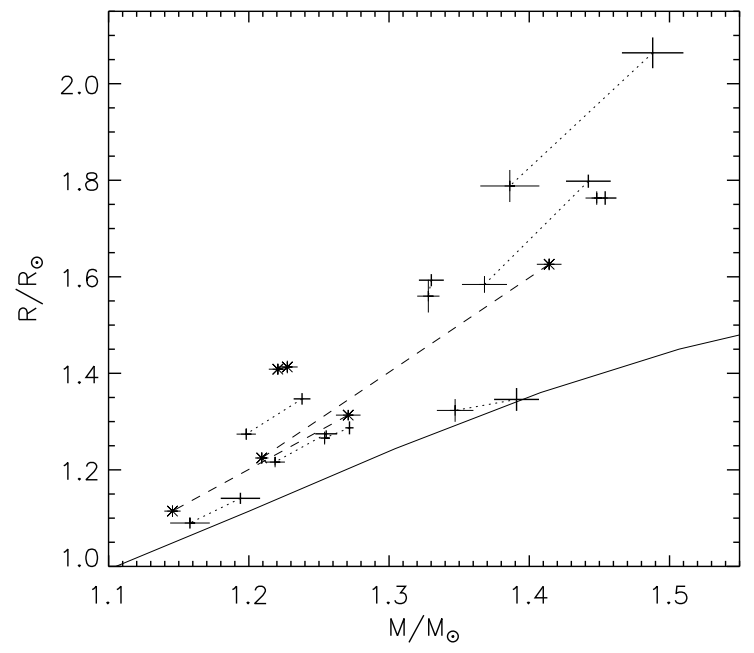

Fig. 1. Main-sequence eclipsing binaries with both components in the 1.1-1.5 $M_{\odot}$ range having masses and radii accurate to $2 \%$ or better. The nine systems shown are, in order of increasing mass of the primary component: EW Ori, UX Men, HS Hya, V505 Per, IT Cas, V1143 Cyg, CD Tau, DM Vir, and BW Aqr. WZ Oph, VZHya, and AD Boo, are shown with star symbols. The full drawn line is the $Y^{2} 0.3 \mathrm{Gyr}$ isochrone for $Z=0.0181$ (Demarque et al. 2004).

affect the observational determination of stellar ages, in particular due to difficulties and shortcomings related to the physics of core overshoot. Moreover, these ages cover the range of interest in studies of the evolution of the Galactic disk (e.g. Holmberg et al. 2007).

In this paper we present new analyses of the three detached F-type double-lined eclipsing binaries AD Boo, VZ Hya, and WZ Oph. From earlier studies they are known to have masses in the range $1.15-1.41 M_{\odot}$ and radii corresponding to the lower half of the main-sequence band, but better accuracy and - especially - abundance data are needed for meaningful tests of state-of-the art stellar models. We have therefore obtained new radial velocity observations, light curves, standard photometry, and high-resolution spectra covering a wide wavelength range, enabling us to determine all relevant parameters of the systems with high precision.

As pointed out by Popper (1998a), and also seen in Fig. $1^{1}$, AD Boo is of particular interest for having components with the greatest differences in mass and radius among well-studied binaries in its mass range. Hence, it may provide stringent tests of stellar models, but the masses obtained in two recent analyses of the system disagree by slightly more than their stated errors (Lacy 1997a; Popper 1998a). Moreover, in both studies the radii were based on reanalyses of the relatively old $B, V$ light curves (normal points) by Zhai et al. (1982), which have an accuracy of only about $0.02 \mathrm{mag}$ and show out-of-eclipse variations by about $0.075 \mathrm{mag}$.

Previous determinations of the dimensions of VZ Hya and WZ Oph were also based on old material (Popper 1965) and therefore had an accuracy of only 2-4\%; see Popper (1980). As in the case of AD Boo, the components of VZ Hya are fairly different, whereas those of WZ Oph are nearly identical.

The paper is structured as follows: First, Sect. 2 describes our observations and analysis techniques adopted. The spectroscopic and photometric analyses of the individual systems are then discussed in detail in Sect. 3. The resulting new, accurate absolute dimensions and abundances are presented in Sect. 4 and

${ }^{1}$ http://www.astro.keele.ac.uk/ jkt/
Table 1. Log of the FEROS observations of AD Boo, VZHya, and WZ Oph. HJD refers to mid-exposure. The exposure time $t_{\text {exp }}$ is given in seconds. The signal-to-noise ratio per resolution element $(S / N)$ of individual exposures was measured around $6070 \AA$ A. Observers: $\mathrm{H}=$ Heidelberg/Copenhagen guaranteed time; JVC = J.V. Clausen; JS = J. Southworth.

\begin{tabular}{ccccc}
\hline \hline HJD-2 400000 & Observer & Phase & $t_{\text {exp }}$ & $S / N$ \\
\hline AD Boo & & & & \\
51207.8746 & JVC & 0.6844 & 2280 & 125 \\
51208.8792 & JVC & 0.1700 & 2400 & 200 \\
VZ Hya & & & & \\
51207.6144 & JVC & 0.2192 & 1800 & 165 \\
51207.7489 & JVC & 0.2655 & 1800 & 160 \\
51210.7054 & JVC & 0.2835 & 2400 & 200 \\
51211.7137 & JVC & 0.6307 & 2400 & 240 \\
51563.8019 & JVC & 0.8606 & 2400 & 160 \\
WZ Oph & & & & \\
51390.6069 & H & 0.3318 & 2700 & 210 \\
52776.7735 & JS & 0.6727 & 300 & 50 \\
52776.8016 & JS & 0.6794 & 300 & 45 \\
52776.8338 & JS & 0.6871 & 300 & 55 \\
52776.8736 & JS & 0.6966 & 300 & 50 \\
52776.9054 & JS & 0.7042 & 300 & 45 \\
52777.6218 & JS & 0.8754 & 300 & 55 \\
52777.7127 & JS & 0.8972 & 300 & 45 \\
52777.7649 & JS & 0.9096 & 300 & 75 \\
52777.8076 & JS & 0.9198 & 300 & 70 \\
52777.8569 & JS & 0.9316 & 300 & 55 \\
52778.7325 & JS & 0.1409 & 300 & 30 \\
52778.7710 & JS & 0.1501 & 420 & 50 \\
52778.8148 & JS & 0.1606 & 300 & 35 \\
\hline & & & &
\end{tabular}

compared with three recent grids of stellar evolutionary models in Sect. 5.

Throughout the paper, the component eclipsed at the deeper eclipse at phase 0.0 is referred to as the primary $(p)$ component, the other stars as the secondary $(s)$.

\section{Observations and analysis techniques}

\subsection{Spectroscopic observations}

The radial velocity observations for the three binaries were conducted at the Harvard-Smithsonian Center for Astrophysics (CfA) with nearly identical echelle spectrographs on the 1.5-m Wyeth reflector at the Oak Ridge Observatory (Harvard, Massachusetts), the 1.5-m Tillinghast reflector at the Whipple Observatory (Mt. Hopkins, Arizona), and the Multiple Mirror Telescope (also on Mt. Hopkins) prior to its conversion to a monolithic mirror. A single echelle order $45 \AA$ wide was recorded with intensified photon-counting Reticon detectors, at a central wavelength of approximately $5187 \AA$, which includes the $\mathrm{Mg} \mathrm{I} \mathrm{b}$ triplet. The resolving power of these instruments is $\lambda / \Delta \lambda \approx 35000$, and the signal-to-noise $(S / N)$ ratios achieved per resolution element of $8.5 \mathrm{~km} \mathrm{~s}^{-1}$ range from 10 to 43 for AD Boo, 15 to 39 for VZHya, and 10 to 25 for WZ Oph. As seen in Figs. 4, 9, and 14, the observations cover the orbits well.

For detailed abundance studies, we have obtained highresolution spectra at higher $S / N$ ratios with the FEROS fiber echelle spectrograph at the ESO 1.52-m and 2.2-m telescopes at La Silla, Chile (Kaufer et al. 1999, 2000). The spectrograph, which resides in a temperature-controlled room, covers without interruption the spectral region from the Balmer jump to $8700 \AA$, at a constant velocity resolution of $2.7 \mathrm{~km} \mathrm{~s}^{-1}$ per pixel $(\lambda / \Delta \lambda=48000)$. An observing log is given in Table 1. 


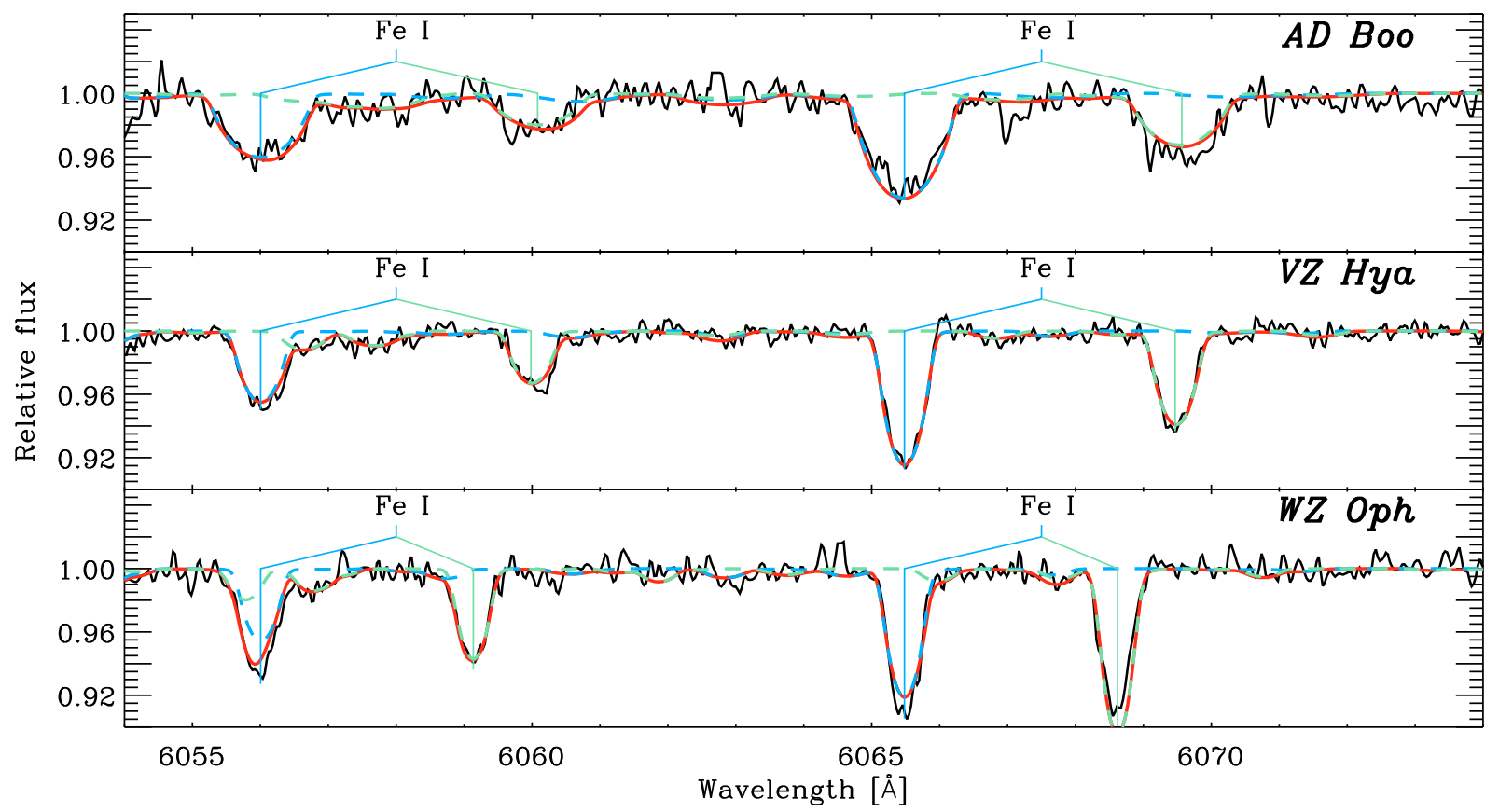

Fig. 2. FEROS spectra (black line) of AD Boo (phase 0.17), VZ Hya (phase 0.28), and WZ Oph (phase 0.33) in the region of the Fe I lines at 6056.0 and $6065.5 \AA$, shifted to laboratory wavelengths for the primary components. Synthetic spectra have been included: primary components (dashed blue); secondary components (dashed green); combined (continuous red line).

A modified version of the MIDAS FEROS package, prepared by Hensberge, was used for the basic reduction of the FEROS spectra ${ }^{2,3}$. Compared to the standard package, background removal, definition and extraction of the individual orders, and wavelength calibration are significantly improved. The observations were reduced night by night using calibration exposures (ThAr and flat field) obtained during the afternoon/morning. Standard (rather than optimal) extraction was applied, and no order merging was attempted. Typically, a standard error of the wavelength calibration of $0.002-0.003 \AA$ was obtained. Subsequently, dedicated $\mathrm{IDL}^{4}$ programs were applied to remove cosmic ray events and other defects, and for normalization of the individual orders. For each order, only the central part with acceptable signal-to-noise ratios was kept for further analysis. A short region of sample FEROS spectra is shown in Fig. 2.

\subsection{Radial velocity determinations}

Radial velocities were obtained from the CfA spectra using the two-dimensional cross-correlation algorithm TODCOR (Zucker $\&$ Mazeh 1994). Templates were selected from a library of synthetic spectra based on model atmospheres by Kurucz (see also Nordström et al. 1994; Latham et al. 2002), calculated over a wide range in effective temperature $\left(T_{\text {eff }}\right)$, rotational velocity $(v \sin i)$, surface gravity $(\log g)$, and metallicity $([\mathrm{M} / \mathrm{H}])$. The templates producing the best match to each component of the binary were identified by running extensive grids of crosscorrelations and selecting the combination giving the highest correlation value averaged over all exposures, weighted according to the strength of each spectrum (e.g. Torres et al. 2002). These grids were in general run in temperature and rotational

\footnotetext{
${ }^{2}$ http://www.ls.eso.org/lasilla/sciops/2p2/E2p2M/ FEROS/DRS

${ }^{3}$ http://www.ls.eso.org/lasilla/sciops/2p2/E1p5M/ FEROS/Reports

${ }^{4}$ http://www.ittvis.com/idl/index.asp
}

velocity only, since those are the parameters that affect the velocities the most. Surface gravities were fixed according to preliminary estimates. If needed, grids for different assumed heavy element abundances were compared, leading indirectly to a rough estimate of $[\mathrm{M} / \mathrm{H}]$ for the system, which was in turn compared with the results from the detailed abundance analysis.

The stability of the zero-point of our velocity system was monitored by means of exposures of the dusk and dawn sky, and small run-to-run corrections were applied as described by Latham (1992). In addition to the radial velocities we derived the light ratio at the mean wavelength of our observations (5187 $\AA$ ), following Zucker \& Mazeh (1994).

Because of the narrow wavelength coverage of the CfA spectra, the radial velocities were checked for systematic errors that might occur as a result of lines of the stars moving in and out of the spectral window with orbital phase (Latham et al. 1996). Numerical simulations were carried out as described in more detail by Torres et al. (1997), and corrections based on them were applied to the raw velocities. These corrections are included in the measurements reported in Tables A.1-A.3.

\subsection{Photometric observations}

uvby light curves for AD Boo, VZ Hya, and WZ Oph were obtained with the Strömgren Automatic Telescope (SAT) at ESO, La Silla during several campaigns between March 1988 and March 1997. They contain 652, 1180, and 697 points in each band, respectively, and cover all phases well. The accuracy per point is 4-5 mmag in $v b y$ and 6-7 mmag in $u$, far better than previous photometry of these systems. The light curves are kept in the stable $u v b y$ instrumental system of the SAT in order not to introduce additional scatter from transformation to the standard uvby system.

Improved linear ephemerides have been calculated for all three systems from the new and numerous published times of minima. No signs of period changes were detected, and the 
Table 2. Effective temperatures $(\mathrm{K})$ of the "average" components of AD Boo, VZHya, and WZ Oph. The $V_{0}$ magnitudes and the $(b-y)_{0}$ and $c_{0}$ indices are based on the out-of-eclipse $u v b y$ standard indices from CVG08. The 2MASS observations $\left(J, H, K_{\mathrm{s}}\right)$ were obtained at phases 0.216 (AD Boo), 0.181 (VZ Hya), and 0.495 (WZ Oph), respectively. For WZOph, the $V$ magnitude was calculated from the value outside eclipses and the $y$ eclipse depth at phase $0.495 . A_{\mathrm{V}}$ is the adopted visual interstellar absorption. References are: A96, Alonso et al. (1996); H07, Holmberg et al. (2007); RM05, Ramírez \& Meléndez (2005); M06, Masana et al. (2006). The results from A96 are based on their uvby calibration, those from RM05 on their uvby, $(V-J),(V-H)$, and $\left(V-K_{\mathrm{s}}\right)$ calibrations (in that order); the calibration by M06 is for $\left(V-K_{\mathrm{s}}\right)$.

\begin{tabular}{lccccccccccc}
\hline \hline System & $A_{\mathrm{V}}$ & {$[\mathrm{Fe} / \mathrm{H}]$} & $(b-y)_{0}$ & $c_{0}$ & $(V-J)_{0}$ & $(V-H)_{0}$ & $\left(V-K_{\mathrm{s}}\right)_{0}$ & $\mathrm{~A} 96$ & $\mathrm{H} 07$ & RM05 & M06 \\
\hline AD Boo & 0.107 & +0.10 & 0.297 & 0.426 & 0.915 & 1.141 & 1.182 & 6456 & 6448 & $6524 / 6370 / 6197 / 6296$ & 6333 \\
VZ Hya & 0.086 & -0.20 & 0.280 & 0.385 & 0.817 & 1.036 & 1.096 & 6511 & 6517 & $6528 / 6707 / 6463 / 6480$ & 6513 \\
WZ Oph & 0.141 & -0.27 & 0.333 & 0.369 & 0.979 & 1.176 & 1.263 & 6115 & 6136 & $6097 / 6190 / 6191 / 6176$ & 6210 \\
\hline
\end{tabular}

orbits were assumed to be circular since there is no evidence for eccentricity, either in previous data or in our own.

In addition to the light curves, homogeneous standard $u v b y \beta$ indices have been established from SAT observations on dedicated nights in which the binaries (and comparison stars) were observed together with a large sample of $u v b y$ and $\beta$ standard stars.

We refer to Clausen et al. (2008, hereafter CVG08) for further details on the photometry and the new ephemerides.

\subsection{Photometric analyses}

The choice of binary model for the light curve analysis depends strongly on the relative radii of the components and the irradiation between them. In our case, the systems are well-detached with relative radii between 0.10 and 0.17 , the components are little deformed (oblateness between 0.001 and 0.007), and reflection effects are small. Therefore, the simple Nelson-Davis-Etzel model (Nelson \& Davis 1972; Etzel 1981; Martynov 1973), which represents the deformed stars as biaxial ellipsoids and applies a simple bolometric reflection model, is expected to be perfectly adequate. We have adopted the corresponding EBOP code (Popper \& Etzel 1981), supplemented by an extended version JKTEBOP ${ }^{5}$ including Monte Carlo simulations (see e.g. Southworth et al. 2004a,b), for the analyses of the three systems and the assignment of realistic errors for the photometric elements.

Since the accuracy of the light curves is high, we have also checked for model-dependent systematic errors in the photometric elements by performing parallel analyses with two more advanced binary models, WINK (Wood 1972) and the widely used Wilson-Devinney (WD) model (Wilson \& Devinney 1971; Wilson 1993, 1994). WINK uses tri-axial ellipsoids, whereas WD is based on Roche geometry. Our WINK version is modified and extended as described by Vaz (1984, 1986), Vaz \& Nordlund (1985), and Nordlund \& Vaz (1990). Our WD code is a revised and extended version of the 1994 code by Wilson; see Vaz et al. (1995) and Casey et al. (1998). In both codes we use the predictions of ATLAS9 model atmospheres (Kurucz 1993) to connect the flux ratios of the two components in different passbands.

In their publicly available versions, EBOP, WINK, and WD carry out a least-squares optimization of the parameters by means of the classical differential corrections method. In JKTEBOP, this has been replaced by the Levenberg-Marquardt minimization algorithm (MRQMIN: Press et al. 1992).

Throughout this paper we have analysed the uvby light curves independently, and for each band identical weights have been assigned to all observations. A photometric scale factor (the magnitude at quadrature) was always included as an adjustable parameter, and the phase of primary eclipse was allowed

\footnotetext{
${ }^{5}$ http://www. astro.keele.ac.uk/ jkt/
}

to shift from 0.0. The mass ratio between the components was kept at the spectroscopic value, and synchronous rotation was assumed. Gravity darkening coefficients/exponents corresponding to convective atmospheres were applied. The bolometric reflection albedo coefficients were fixed at 0.5 in the WINK and WD analyses, again due to convection; for EBOP the simple bolometric reflection model was used.

Linear limb-darkening coefficients were either assigned from theoretical calculations (van Hamme 1993; Díaz-Cordovés et al. 1995; Claret 2000) according to the effective temperatures, surface gravities, and abundances, or included as adjustable parameters. For all components of the three systems, the linear coefficients by Claret (2000) are about 0.10 higher than those by van Hamme (1993) and about 0.03 higher than those by Díaz-Cordovés et al. (1995). In general, a change in the coefficients by 0.10 causes systematic differences between the optimum theoretical light curves of just a few mmag through the eclipses, and the overall quality of the fits to the observations is nearly identical. If adjusted, the coefficients for the components of VZHya and WZOph turn out closest to the values by van Hamme; for AD Boo the coefficients either become unrealistic or convergence is not achieved at all. In addition, a few WD analyses with the non-linear law by Klinglesmith \& Sobieski (1970) and coefficients by van Hamme (1993) were included. No improvements in the light curve fits were seen. The influence on the other photometric elements of adopting different limb-darkening coefficients/laws are discussed below for the individual systems. In order to include uncertainties of the limb-darkening coefficients in the uncertainties of the final photometric elements, random variations within $+/-0.05$ have been introduced in the JKTEBOP Monte Carlo simulations mentioned above.

In tables and text on photometric solutions we use the following symbols: $i$ orbital inclination; $r$ relative radius; $k=r_{\mathrm{s}} / r_{\mathrm{p}}$; $u$ linear limb darkening coefficient; $y$ gravity darkening coefficient; $J$ central surface brightness; $L$ luminosity; $\Omega$ surface potential; $T_{\text {eff }}$ effective temperature.

\subsection{Effective temperatures and interstellar reddening}

Although significantly improved (semi)empirical temperature scales and calibrations have become available during the last decade, the determination of effective temperatures of binary components remains a difficult task; see e.g. Popper (1980), Andersen (1991), and Clausen (2004) for general remarks. For comparison with theoretical models, luminosity calculations, and distance determinations, it is on the other hand important to diminish both systematic and random errors as much as possible. In the analyses of AD Boo, VZ Hya, and WZ Oph we have chosen to follow three independent approaches:

- First, as part of the light curve analyses we calculate the individual $u v b y$ indices for the components from the combined 
standard $u v b y$ indices (CVG08) and the luminosity ratios between the components in the four bands (based on mean geometric elements). Interstellar reddening is determined from the intrinsic colour calibration by Olsen (1988), which has a precision of $0.009 \mathrm{mag}$, and the results are compared to those from other sources. Effective temperatures can then be obtained from a series of (semi)empirical calibrations such as those by Alonso et al. (1996), Ramírez \& Meléndez (2005), and Holmberg et al. (2007). Also, as an important check on these calibrations, effective temperature differences between the components can be derived from the empirical flux calibration by Popper (1980) and the $y$ surface flux ratios. They are obtained to high precision in the light curve analyses, since they depend mainly on the observed depths of primary and secondary eclipses. We have applied both the BC scale used by Popper (1980) and that by Flower (1996), which in the temperature range of AD Boo, VZ Hya, and WZ Oph, lead to nearly identical temperature differences with errors of only about $25 \mathrm{~K}$ (excluding uncertainties in Popper's flux scale).

- Second, we attempt to obtain spectroscopic temperatures from the high-resolution and high- $S / N$ spectra, either combined or disentangled, as part of the abundance analyses; see e.g. Santos et al. (2004) and Bruntt et al. (2004) for details. And thirdly, as mentioned in Sect. 2.2, temperatures are estimated by identifying the templates producing the best match to each component in the TODCOR analyses, and interpolating in $\log g$ or metallicity as necessary. As discussed below for the individual systems, the two latter methods however tend to be problematic.

- Finally, the photometric indices of the combined light of a system may provide a first estimate of the effective temperature of its "average" component, and if the components are nearly identical, as is the case for WZ Oph, a direct determination is possible. In Table 2 we present such temperatures derived from the combined $u v b y$ indices and $2 \mathrm{MASS}^{6} \mathrm{JHK}_{\mathrm{s}}$ observations. As seen, the temperatures from the different $u v b y$ and $V J H K_{\mathrm{s}}$ calibrations agree well for WZOph. The spread for AD Boo and VZ Hya presumably reflects the fact that their combined flux distributions are distorted compared to a single star, because the components differ by 400-600 K in effective temperature.

\subsection{Abundance analyses}

For meaningful comparisons between theoretical stellar models and empirical absolute dimensions - as determined from detached double-lined eclipsing binaries - the chemical composition, and in particular the metal abundance, must be known for the binary. However, detailed abundance studies have only been done for a few systems. As part of the analyses of AD Boo, VZ Hya, and WZ Oph, we have used the new spectroscopic and photometric data to establish their metal abundance. Several methods were applied/attempted:

1. spectroscopic analyses of observed or disentangled FEROS spectra;

2. spectroscopic analyses of disentangled CfA spectra;

3. template matching as part of the TODCOR analyses;

4. photometric $u v b y-[\mathrm{Fe} / \mathrm{H}]$ calibrations.

\footnotetext{
${ }^{6}$ http://www.ipac. caltec. edu/2mass
}

\subsubsection{FEROS spectra}

The FEROS spectra (Table 1) of WZ Oph were disentangled in order to extract the individual component spectra. We applied the disentangling method introduced by Simon \& Sturm (1994) and a revised version of the corresponding original code developed by Sturm. It assumes a constant light level, so only spectra taken outside of eclipse can be included. We note that all three systems are in fact constant to within a few percent outside of eclipse. The orbital elements were fixed at the results based on the TODCOR analyses of the CfA spectra. For AD Boo, with only two FEROS spectra available, disentangling is not possible. Disentangling of the five VZ Hya spectra was attempted, but the quality of the resulting component spectra was not acceptable; more than five spectra and/or a better phase distribution seem to be needed. For both systems the observed double-lined spectra were therefore used directly for the abundance analyses.

The versatile semi-automatic IDL tool $\mathrm{VWA}^{7}$, developed by Bruntt, was applied for the abundance analyses. We refer to Bruntt et al. (2002, 2004, 2008) for further details. For our particular study, VWA has been extended to allow the analysis of double-lined spectra in addition to normal spectra from single stars - or disentangled binary component spectra. We have adopted the effective temperatures, surface gravities, and rotational velocities determined for the binary components in this study (Table 21), but for comparison analyses for higher or lower temperatures were also done. Microturbulence velocities were derived from the calibration by Edvardsson et al. (1993), which has a scatter of about $0.3 \mathrm{~km} \mathrm{~s}^{-1}$, but have been tuned, if needed, as part of the analyses.

In each spectrum, VWA selects the least blended lines from atomic line lists extracted from the Vienna Atomic Line Database (VALD; Kupka et al. 1999). For each selected line, the synthetic spectrum is then calculated, and the input abundance is changed iteratively until the equivalent width of the observed and synthetic lines match. The synthetic spectra are generated with the SYNTH software (Valenti \& Piskunov 1996). Atmosphere models were either interpolated from the grid of modified ATLAS9 models by Heiter et al. (2002) or calculated from the MARCS code. We have applied an improved version of the original model by Gustafsson et al. (1975); see Jørgensen et al. (1992) for further details.

For the binary components, we calculate all abundances relative to the Sun, line by line. We have used either an atlas spectrum of the solar flux (Wallace et al. 1998) or a FEROS sky spectrum. In general, this approach gives better agreement between the abundances determined from the individual lines of a given element/ion.

If we adopt the VALD line data and the solar photosphere abundances by Grevesse \& Sauval (1998), we obtain for the Sun the Fe abundances listed in Table 3 for the solar atlas and FEROS spectra. Only lines with equivalent widths between 10 and $80 \mathrm{~m} \AA$ measured in both spectra were included. It is gratifying to see that the results from the FEROS spectrum agree very well with those from the atlas spectrum. For both spectra, Fe I and Fe II lines give slightly different $[\mathrm{Fe} / \mathrm{H}]$ results. They agree a bit better for the MARCS models than for ATLAS9 models, and the MARCS models also reproduce the solar result slightly better.

Although we will focus on Fe, we have also analysed other elements, and results from the solar atlas spectrum are given in Table 4. For most of the ions with many measured lines we

\footnotetext{
7 http://www.hans.bruntt.dk/vwa/
} 
Table 3. Iron abundance $([\mathrm{Fe} / \mathrm{H}])$ for the Sun determined from Solar atlas spectrum (Wallace et al. 1998) and FEROS spectra, respectively. $131 \mathrm{Fe}$ I and $20 \mathrm{Fe}$ II lines, measured in both spectra, were used. Results from ATLAS9 models and MARCS models are compared.

\begin{tabular}{lcc}
\hline \hline & Fe I & Fe II \\
\hline ATLAS9: & & \\
Solar atlas & $+0.11 \pm 0.10$ & $-0.02 \pm 0.07$ \\
FEROS & $+0.11 \pm 0.10$ & $-0.04 \pm 0.07$ \\
MARCS: & & \\
Solar atlas & $+0.07 \pm 0.09$ & $+0.00 \pm 0.10$ \\
FEROS & $+0.07 \pm 0.10$ & $-0.01 \pm 0.09$ \\
\hline
\end{tabular}

Table 4. Abundances for the Sun determined from Solar atlas spectrum (Wallace et al. 1998) and ATLAS9 models (Heiter et al. 2002). $N$ is the number of lines used; only ions with three or more measured lines are listed. GS1998 is the logarithmic element abundances $(H=12.00)$ from Grevesse \& Sauval (1998).

\begin{tabular}{lrrrr}
\hline \hline Ion & [El./H] & rms & $N$ & GS1998 \\
\hline C I & 0.21 & 0.12 & 7 & $8.52 \pm 0.06$ \\
O I & 0.00 & 0.16 & 4 & $8.83 \pm 0.06$ \\
Na I & 0.03 & 0.07 & 9 & $6.33 \pm 0.03$ \\
Mg I & -0.08 & 0.23 & 14 & $7.58 \pm 0.05$ \\
Al I & -0.17 & 0.11 & 4 & $6.47 \pm 0.07$ \\
Si I & -0.17 & 0.29 & 38 & $7.55 \pm 0.05$ \\
Si II & 0.12 & 0.33 & 3 & $7.55 \pm 0.05$ \\
S I & 0.11 & 0.21 & 5 & $7.33 \pm 0.11$ \\
Ca I & 0.06 & 0.19 & 25 & $6.36 \pm 0.02$ \\
Sc I & -0.11 & 0.14 & 8 & $3.17 \pm 0.10$ \\
Sc II & 0.06 & 0.09 & 15 & $3.17 \pm 0.10$ \\
Ti I & -0.02 & 0.07 & 34 & $5.02 \pm 0.06$ \\
Ti II & 0.06 & 0.12 & 21 & $5.02 \pm 0.06$ \\
V I & 0.00 & 0.13 & 52 & $4.00 \pm 0.02$ \\
V II & 0.32 & 0.28 & 4 & $4.00 \pm 0.02$ \\
Cr I & 0.07 & 0.12 & 36 & $5.67 \pm 0.03$ \\
Cr II & 0.16 & 0.15 & 22 & $5.67 \pm 0.03$ \\
Mn I & 0.19 & 0.22 & 39 & $5.39 \pm 0.03$ \\
Fe I & 0.11 & 0.10 & 238 & $7.50 \pm 0.05$ \\
Fe II & -0.02 & 0.08 & 30 & $7.50 \pm 0.05$ \\
Co I & 0.03 & 0.18 & 49 & $4.92 \pm 0.04$ \\
Ni I & 0.03 & 0.10 & 93 & $6.25 \pm 0.04$ \\
Cu I & -0.17 & 0.27 & 4 & $4.21 \pm 0.04$ \\
Zn I & 0.09 & 0.13 & 4 & $4.60 \pm 0.08$ \\
Y II & 0.08 & 0.17 & 14 & $2.24 \pm 0.03$ \\
Zr I & -0.23 & 0.07 & 3 & $2.60 \pm 0.02$ \\
Ba II & 0.23 & 0.08 & 3 & $2.13 \pm 0.05$ \\
Ce II & 0.11 & 0.21 & 8 & $1.58 \pm 0.09$ \\
Nd II & 0.13 & 0.19 & 5 & $1.50 \pm 0.06$ \\
Sm II & 0.05 & 0.23 & 3 & $1.01 \pm 0.06$ \\
\hline & & & &
\end{tabular}

obtain good agreement with Grevesse \& Sauval (1998), but there are exceptions such as Si I, Cr II, and Mn I. This may indicate errors in the VALD line data (a problem with Mn was found by Bruntt et al. 2004) which should, however, be eliminated to a large extent in the binary abundance analyses performed relative to the Sun. Comparison with the most recent solar photosphere abundances by Grevesse et al. (2007), which for many elements are derived using updated line data, 3D models and departures from LTE, is less obvious, since we apply 1D, LTE atmosphere codes.

\subsubsection{Disentangled CfA spectra}

As seen in Tables 5, 11, and 16, a large number of CfA spectra are available for all three systems. We have selected those
Table 5. Spectroscopic orbital solution for AD Boo. $T$ is the time of central primary eclipse.

\begin{tabular}{lr}
\hline \hline Parameter & \multicolumn{1}{c}{ Value } \\
\hline Adjusted quantities: & \\
$K_{\mathrm{p}}\left(\mathrm{km} \mathrm{s}^{-1}\right)$ & $106.13 \pm 0.19$ \\
$K_{\mathrm{s}}\left(\mathrm{km} \mathrm{s}^{-1}\right)$ & $124.11 \pm 0.36$ \\
$\gamma\left(\mathrm{km} \mathrm{s}^{-1}\right)$ & $-16.52 \pm 0.13$ \\
Adopted quantities: & \\
$P($ days $)$ & 2.06880704 \\
$T$ (HJD-2 400 000) & 49311.43169 \\
$e$ & 0.00 \\
Derived quantities: & \\
$M_{\mathrm{p}} \sin ^{3} i\left(M_{\odot}\right)$ & $1.4103 \pm 0.0089$ \\
$M_{\mathrm{s}} \sin ^{3} i\left(M_{\odot}\right)$ & $1.2061 \pm 0.0056$ \\
$a_{\mathrm{p}} \sin i\left(10^{6} \mathrm{~km}\right)$ & $3.0193 \pm 0.0053$ \\
$a_{\mathrm{s}} \sin i\left(10^{6} \mathrm{~km}\right)$ & $3.5307 \pm 0.0103$ \\
$a \sin i\left(R_{\odot}\right)$ & $9.411 \pm 0.017$ \\
Other quantities pertaining to the fit: & \\
$N_{\text {obs }}$ & 124 \\
Time span (days) & 3074 \\
$\sigma_{\mathrm{p}}\left(\mathrm{km} \mathrm{s}^{-1}\right)$ & 1.63 \\
$\sigma_{\mathrm{s}}\left(\mathrm{km} \mathrm{s}^{-1}\right)$ & 3.15 \\
\hline
\end{tabular}

collected outside eclipses with sufficient $S / N$ ratios for disentangling. The actual numbers of spectra used are 113 (AD Boo), 29 (VZ Hya), and 40 (WZ Oph). After careful normalization of the individual spectra, the disentangled component spectra were constructed by adopting the orbital elements listed in the tables mentioned above. The luminosity ratios between the components, which cannot be determined from the disentangling procedure, were calculated by interpolation between the $b$ and $y$ luminosity ratios of the photometric solutions. The disentangled component spectra were then compared to the CfA library of synthetic spectra mentioned in Sect. 2.2.

In general we find that the short wavelength range covered (45 $\AA$ ), and the correspondingly small number of lines available, limits the success of this approach. Most importantly, it was difficult to break the correlation between metal abundance and effective temperature. Results for the individual systems will, however, be presented below.

\subsubsection{TODCOR template matching}

As mentioned in Sect. 2.2, rough estimates of the metal abundance for a system can be obtained directly from the observed composite spectra by running extensive grids of twodimensional cross-correlations (TODCOR) using templates of different metallicity. Results from this exercise will also be discussed below for the individual systems.

\subsection{4. uvby calibration}

Finally, we have derived metal abundances from the uvby indices for the individual binary components (Table 21) and the recent calibrations by Holmberg et al. (2007), which are based on a carefully selected sample of spectroscopic $[\mathrm{Fe} / \mathrm{H}]$ results for several hundred stars. The calibration adopted for $0.30<$ $b-y<0.46$ has a dispersion of only $0.07 \mathrm{dex}$; that for bluer stars has a dispersion of 0.10 dex. Taking into account the uncertainties of the uvby indices and the interstellar reddening, an overall precision of 0.10 to 0.15 dex can be achieved per component. 


\section{Spectroscopic and photometric analyses}

In this section we present in detail the spectroscopic and photometric analyses of AD Boo, VZ Hya, and WZ Oph, including abundances, and compare our results with those obtained in previous studies. Additional references to earlier work are given by CVG08.

\section{1. $A D B O 0$}

$\mathrm{ADB}$ Boo $=\mathrm{BD}+25^{\circ} 2800=\mathrm{BV} 135(P=2.07)$ was discovered by Strohmeier et al. (1956) to be an eclipsing binary, and Lacy (1985) found it to be double-lined. It has most recently been studied by Lacy (1997a) and Popper (1998a).

\subsubsection{Radial velocities and spectroscopic orbit for AD Boo}

Radial velocities for the components of ADBoo were determined by TODCOR analyses of 124 usable CfA spectra outside of eclipse, observed from January 1989 to June 1997. Surface gravities $(\log g)$ were fixed at 4.0 (primary) and 4.5 (secondary), and the heavy element abundance was assumed to be solar, the closest values in our grid of synthetic spectra to the actual values determined in Sect. 4. We inferred temperatures of $6250 \pm 150 \mathrm{~K}$ for the primary of AD Boo (the more massive star), and $5680 \pm 300 \mathrm{~K}$ for the secondary, both significantly lower than the photometric values, because the templates have lower metallicity than measured for AD Boo; see Sects. 3.1.3 and 4. The measured $v \sin i$ values are $38 \pm 2 \mathrm{~km} \mathrm{~s}^{-1}$ and $37 \pm 5 \mathrm{~km} \mathrm{~s}^{-1}$, respectively, with the secondary being considerably more uncertain due to its much weaker lines. The synchronous values are $39 \mathrm{~km} \mathrm{~s}^{-1}$ for the primary and $30 \mathrm{~km} \mathrm{~s}^{-1}$ for the secondary. Lacy (1997a) reported $38 \pm 1$ and $31 \pm 1 \mathrm{~km} \mathrm{~s}^{-1}$, whereas Popper (1998a) did not give any values. Templates from our library with parameters closest to the measured values were used to derive the radial velocities.

In the case of $\mathrm{AD}$ Boo we found that the radial velocity corrections for systematics described in Sect. 2.2, which are shown in Fig. 3, were quite significant, reaching up to $10 \mathrm{~km} \mathrm{~s}^{-1}$ for the secondary star and up to $2 \mathrm{~km} \mathrm{~s}^{-1}$ for the primary. The effect on the absolute masses is large, $\sim 6 \%$ for the primary and $\sim 4 \%$ for the secondary. The final velocities including corrections are listed in Table A.1. The light ratio derived from the spectra, including also corrections for systematics analogous to those derived for the velocities, is $\ell_{\mathrm{s}} / \ell_{\mathrm{p}}=0.37 \pm 0.02$ at the mean wavelength of our observations (5187 $\AA$ ).

The spectroscopic orbital solution for AD Boo is given in Table 5, and the observations and computed orbit are shown graphically in Fig. 4 along with the residuals for each star. For comparison, we have also disentangled 113 CfA spectra outside of eclipse and obtain velocity semiamplitudes of $K_{\mathrm{p}}=105.95$ and $K_{\mathrm{s}}=124.50$, in good agreement with the results based on TODCOR velocities.

Our velocity semiamplitudes agree well with those by Popper $(1998 \mathrm{a}),\left(K_{\mathrm{p}}, K_{\mathrm{s}}\right)=(105.85 \pm 0.35,124.4 \pm 0.4)$, whereas Lacy (1997a) obtained $\left(K_{\mathrm{p}}, K_{\mathrm{s}}\right)=(107.2 \pm 0.5,124.6 \pm 0.6)$.

\subsubsection{Photometric elements for AD Boo}

The uvby light curves of AD Boo contain 652 observations in each band and were observed on 42 nights during five periods between March 1988 and March 1992 (CVG08). The accuracy per point is about $0.005 \mathrm{mag}(v b y)$ and $0.007 \mathrm{mag}(u)$, but
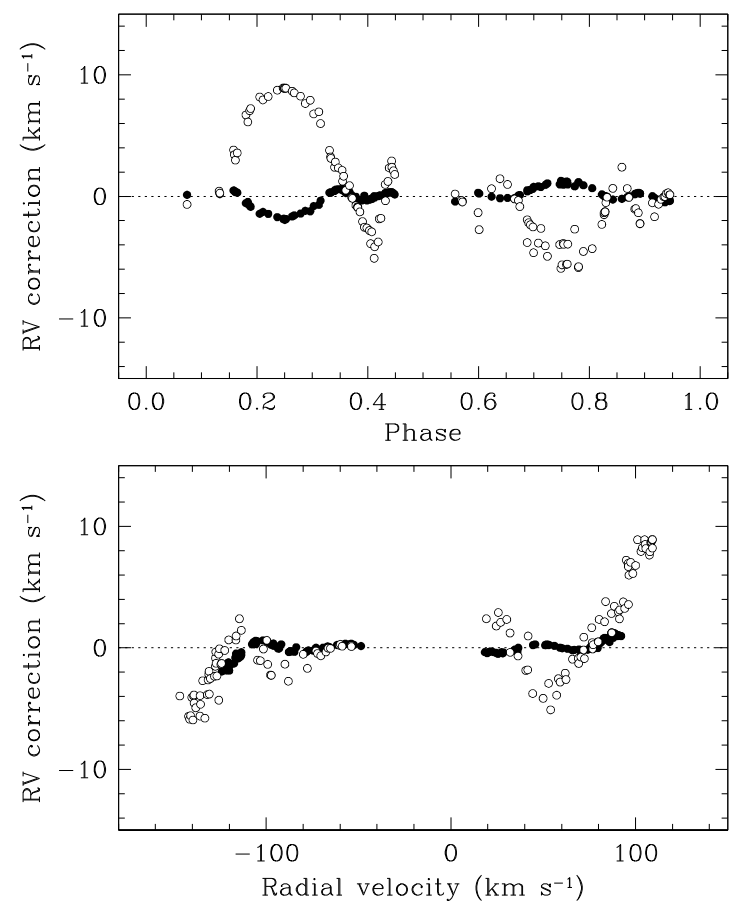

Fig. 3. Systematic errors in the raw TODCOR velocities of ADBoo determined from simulations with synthetic binary spectra (filled circles: primary; open circles: secondary). The differences are plotted both as function of orbital phase (upper panel) and radial velocity (lower panel), and have been applied to the measured velocities as corrections. Phase 0.0 corresponds to central primary eclipse.

comparison of the data from the five periods reveals that at some phases, systematic differences in the light level of 0.01-0.03 mag exist, increasing from $y$ to $u$. In particular, observations during the 1989 eclipses are systematically fainter. The effect is shown in Fig. 5 and indicates surface activity (spots) at a rather low level on one or both components, the cooler secondary being the most likely candidate. Zhai et al. (1982) also noticed scatter in their $B$ and $V$ light curves, which were obtained from April to June 1981, but at a much higher level of about 0.075 mag compared to their observing error of about $0.02 \mathrm{mag}$.

There is very little information on activity in the literature, and AD Boo does not appear to be an X-ray source. The presence of chromospheric activity in terms of the Rossby number ${ }^{8}$ was studied by Hall (1994). The secondary in AD Boo is perhaps cool enough to display significant activity (see above) despite having a mass as high as $1.21 M_{\odot}$, which would imply a relatively thin convective envelope. An estimate of the color index of $B-V=0.58$ based on the temperature allows the convective turnover time to be inferred, and this together with the rotational period (assuming synchronous rotation) leads to a Rossby number of approximately 0.20 , which is in the range where other stars tend to show spot activity (see Hall 1994). Furthermore, our two high-resolution FEROS spectra taken in January 1999 (see Table 1) show in fact a weak emission feature in the $\mathrm{Ca}$ II $\mathrm{H}$ and $\mathrm{K}$ lines at the position of the secondary component, supporting also some activity. Nothing is seen in the $\mathrm{H}_{\alpha}$ line.

EBOP and WD solutions, based on linear limb-darkening coefficients by van Hamme (1993) are presented in Tables 6 and 7, respectively, and $\mathrm{O}-\mathrm{C}$ residuals of the $v$ observations from the theoretical light curve computed for the photometric elements

\footnotetext{
8 Defined as the ratio of the rotation period to the convective turnover time.
} 

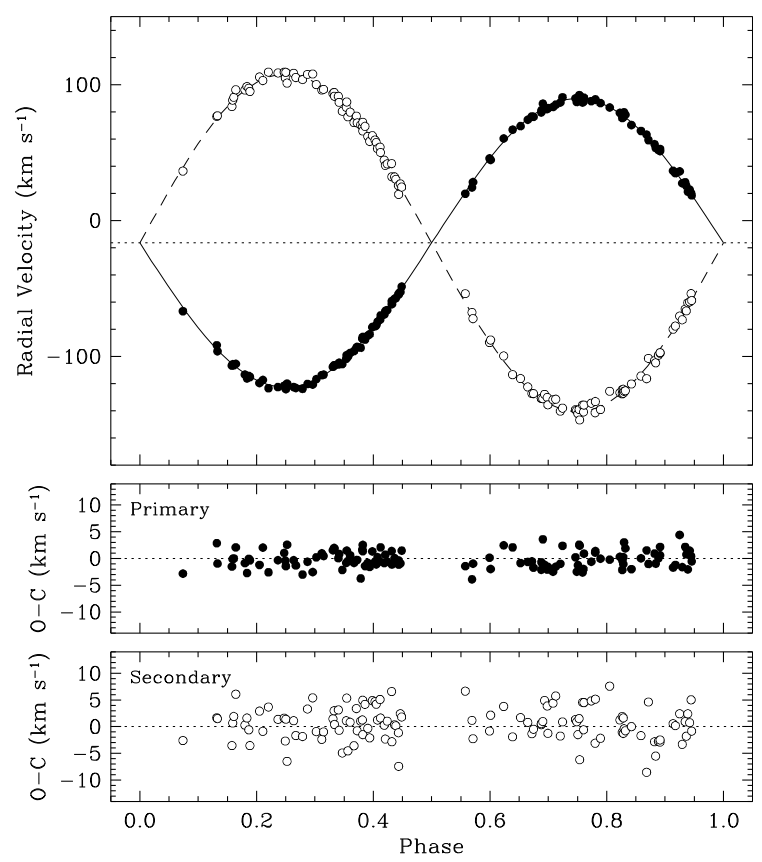

Fig. 4. Spectroscopic orbital solution for AD Boo (solid line: primary; dashed line: secondary) and radial velocities (filled circles: primary; open circles: secondary). The dotted line (upper panel) represents the center-of-mass velocity of the system. Phase 0.0 corresponds to central primary eclipse.

Table 6. Photometric solutions for AD Boo (all data) from the EBOP code adopting linear limb darkening coefficients by van Hamme (1993). The errors quoted for the free parameters are the formal errors determined from the iterative least squares solution procedure.

\begin{tabular}{|c|c|c|c|c|}
\hline & $\bar{y}$ & $b$ & $\bar{v}$ & $u$ \\
\hline \multirow[t]{2}{*}{$i \quad\left(^{\circ}\right)$} & 87.58 & 87.69 & 87.83 & 87.89 \\
\hline & \pm 12 & \pm 10 & \pm 10 & \pm 15 \\
\hline \multirow[t]{2}{*}{$r_{\mathrm{p}}$} & 0.1711 & 0.1715 & 0.1709 & 0.1713 \\
\hline & \pm 5 & \pm 4 & \pm 4 & \pm 7 \\
\hline$r_{\mathrm{s}}$ & 0.1291 & 0.1292 & 0.1289 & 0.1296 \\
\hline \multirow[t]{2}{*}{$k$} & 0.754 & 0.753 & 0.754 & 0.756 \\
\hline & \pm 5 & \pm 4 & \pm 3 & \pm 5 \\
\hline$r_{\mathrm{p}}+r_{\mathrm{s}}$ & 0.3002 & 0.3007 & 0.2998 & 0.3009 \\
\hline$u_{\mathrm{p}}$ & 0.52 & 0.61 & 0.68 & 0.65 \\
\hline$u_{\mathrm{s}}$ & 0.56 & 0.65 & 0.73 & 0.75 \\
\hline$y_{\mathrm{p}}$ & 0.34 & 0.39 & 0.45 & 0.53 \\
\hline$y_{\mathrm{s}}$ & 0.36 & 0.42 & 0.48 & 0.57 \\
\hline \multirow[t]{2}{*}{$J_{\mathrm{s}} / J_{\mathrm{p}}$} & 0.7314 & 0.6888 & 0.6440 & 0.6775 \\
\hline & \pm 33 & \pm 30 & \pm 31 & \pm 48 \\
\hline$L_{\mathrm{s}} / L_{\mathrm{p}}$ & 0.4079 & 0.3828 & 0.3566 & 0.3693 \\
\hline$\sigma \quad(\mathrm{mag})$ & 0.0064 & 0.0063 & 0.0067 & 0.0102 \\
\hline
\end{tabular}

given in Table 6 are shown in Fig. 5. As seen, differences between the photometric elements obtained from the two models are small, and the mean values of $r_{\mathrm{p}}+r_{\mathrm{s}}$ and $k$ agree within their uncertainties. WINK solutions, which are not included, agree well with those from EBOP and WD. Slightly different orbital inclinations are obtained, however, for the four bands from all three codes. This could be due to either the systematic light level differences mentioned above (spots), which increase from $y$ to $u$, to a small amount of (red) third light, or to a slightly incorrect theoretical wavelength dependence of the limb-darkening coefficients.

Excluding the 1989 observations (see Fig. 5), we obtain the EBOP solutions listed in Table 8 . The relative radii change by
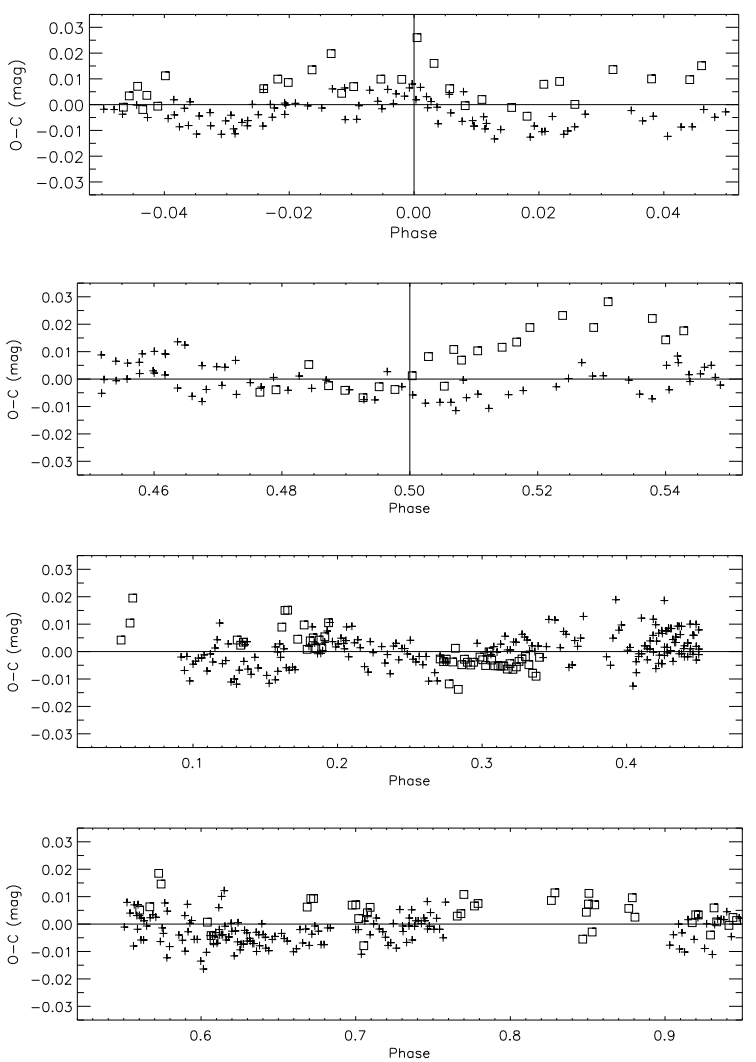

Fig. 5. $(\mathrm{O}-\mathrm{C})$ residuals of the AD Boo $v$-band observations from the theoretical light curve computed for the photometric elements given in Table 6. 1989 observations are shown with square symbols.

Table 7. Photometric solutions for AD Boo (all data) from the WD code adopting linear limb darkening coefficients by van Hamme (1993); see Table 6. Gravity darkening exponents of 0.33 and bolometric albedo coefficients of 0.5 were applied, as appropriate for convective envelopes. $T_{\text {eff,p }}$ was assumed to be $6575 \mathrm{~K}$.

\begin{tabular}{lrrrr}
\hline \hline & $y$ & $b$ & $v$ & $u$ \\
\hline$i\left(^{\circ}\right)$ & 87.47 & 87.78 & 87.79 & 87.82 \\
& \pm 5 & \pm 3 & \pm 3 & \pm 6 \\
$\Omega_{\mathrm{p}}$ & 6.743 & 6.711 & 6.717 & 6.702 \\
& \pm 11 & \pm 8 & \pm 9 & \pm 14 \\
$\Omega_{\mathrm{s}}$ & 7.646 & 7.696 & 7.675 & 7.636 \\
& \pm 16 & \pm 10 & \pm 9 & \pm 18 \\
$r_{\mathrm{p}}$ & 0.1705 & 0.1713 & 0.1711 & 0.1716 \\
$r_{\mathrm{s}}$ & 0.1303 & 0.1293 & 0.1297 & 0.1305 \\
$k$ & 0.764 & 0.755 & 0.758 & 0.761 \\
$r_{\mathrm{p}}+r_{\mathrm{s}}$ & 0.3008 & 0.3006 & 0.3008 & 0.3021 \\
$T_{\text {eff,s }}$ & 6078 & 6052 & 5999 & 5946 \\
& \pm 4 & \pm 4 & \pm 4 & \pm 4 \\
$L_{\mathrm{s}} / L_{\mathrm{p}}$ & 0.4205 & 0.3868 & 0.3615 & 0.3761 \\
$\sigma$ (mag.) & 0.0064 & 0.0062 & 0.0066 & 0.0101 \\
\hline
\end{tabular}

about $0.7 \%$ in opposite directions and their sum by about $-0.6 \%$, whereas the orbital inclination is unchanged in all four bands. Including $2 \%$ of third light changes $i$ by about 0.3 , which is the maximum difference between the four bands, but we have not found any direct support for even such a small amount of third light in any of the analyses. If we adopt linear limb-darkening coefficients from Claret (2000), which are 0.04-0.07 higher, the orbital inclination decreases by 0.16 , and the relative radius increases by about 0.0013 for the primary component but is unchanged for the secondary. If adjusted, the linear limb-darkening 
Table 8. Photometric solutions for AD Boo from the EBOP code, excluding observations from 1989. Linear limb darkening coefficients from van Hamme (1993) were adopted; see Table 6.

\begin{tabular}{lrrrr}
\hline \hline & $y$ & $b$ & $v$ & $u$ \\
\hline$i\left(^{\circ}\right)$ & 87.59 & 87.67 & 87.83 & 87.93 \\
& \pm 13 & \pm 11 & \pm 11 & \pm 17 \\
$r_{\mathrm{p}}$ & 0.1703 & 0.1707 & 0.1698 & 0.1698 \\
& \pm 5 & \pm 5 & \pm 4 & \pm 7 \\
$r_{\mathrm{s}}$ & 0.1284 & 0.1284 & 0.1279 & 0.1279 \\
$k$ & 0.754 & 0.752 & 0.753 & 0.753 \\
& \pm 6 & \pm 4 & \pm 3 & \pm 6 \\
$r_{\mathrm{p}}+r_{\mathrm{s}}$ & 0.2987 & 0.2991 & 0.2977 & 0.2977 \\
$J_{\mathrm{s}} / J_{\mathrm{p}}$ & 0.7339 & 0.6872 & 0.6408 & 0.6707 \\
& \pm 38 & \pm 35 & \pm 35 & \pm 57 \\
$L_{\mathrm{s}} / L_{\mathrm{p}}$ & 0.4090 & 0.3809 & 0.3538 & 0.3627 \\
$\sigma$ (mag.) & 0.0059 & 0.0055 & 0.0056 & 0.0091 \\
\hline
\end{tabular}
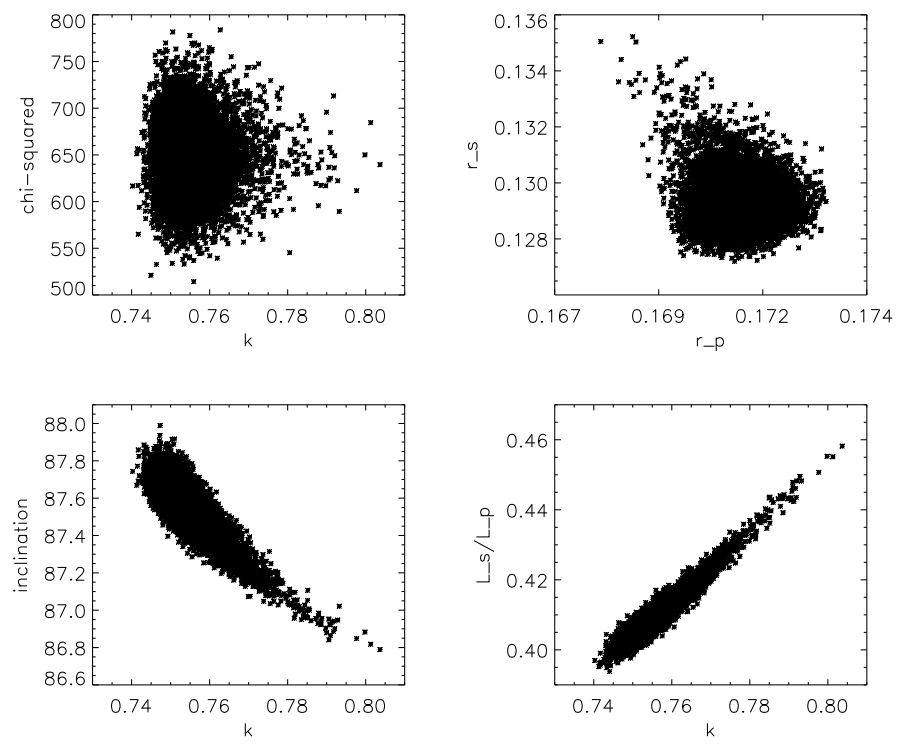

Fig. 6. Best fitting parameter values for the 10000 synthetic AD Boo $y$-band light curves created for the Monte Carlo analysis.

coefficients either become unrealistic and the orbital inclinations from the four bands disagree much more, or convergence fails. Finally, WD solutions including non-linear limb darkening (see Sect. 2.4) give photometric elements very close to those presented in Table 7. We are therefore unable to point at a clear explanation for the small $i$ discrepancies, which are, however, of no consequence for the determination of the absolute properties.

In order to assign realistic errors to the photometric elements, we have supplemented these analyses with 10000 JKTEBOP Monte Carlo simulations in each band; see Fig. 6. Furthermore, as shown in Fig. 7, we have compared EBOP solutions for a range of fixed $k=r_{\mathrm{s}} / r_{\mathrm{p}}$ values near 0.75 . The adopted photometric elements listed in Table 9 are the weighted mean values of the EBOP solutions adopting the linear limb-darkening coefficients by van Hamme. Errors are based on the Monte Carlo simulations and comparison between the uvby solutions, including those where the 1989 observations were excluded. We find that secondary eclipse is in fact total, whereas at phase 0.0 about $61 \%$ of the $y$ light from the primary component is eclipsed. The spectroscopic light ratio of $\ell_{\mathrm{s}} / \ell_{\mathrm{p}}=0.37 \pm 0.02$ at $5187 \AA$ agrees quite well with that from the light curve solutions (0.40).

For comparison, Lacy (1997a), who applied the EBOP code to analyse the $B$ and $V$ normal points by Zhai et al. (1982), obtained $i=87.8 \pm 0.2, r_{\mathrm{p}}=0.1702 \pm 0.0011, k=0.750 \pm 0.010$,
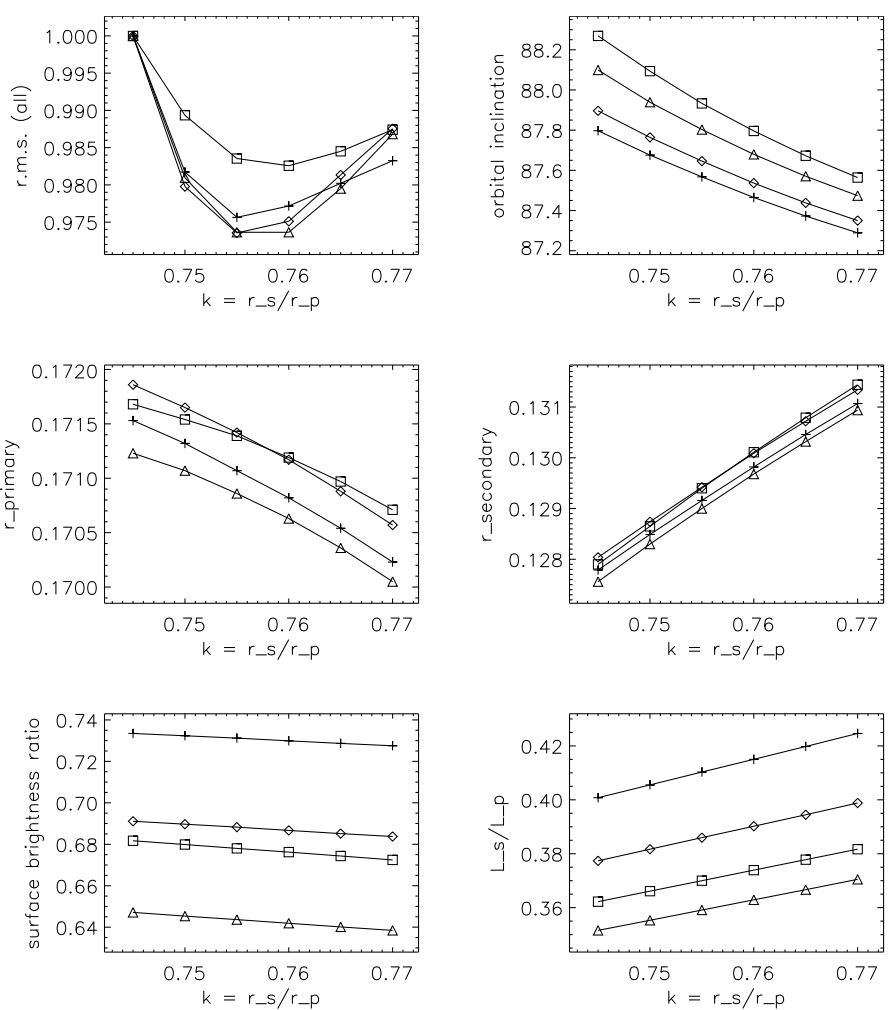

Fig. 7. EBOP solutions for AD Boo for a range of fixed $k$ values. Linear limb-darkening coefficients by van Hamme (1993) were adopted. The upper left panel shows normalized rms errors of the fit to the observations. Symbols are: cross $y$; diamond $b$; triangle $v$; square $u$.

$J_{\mathrm{s}} / J_{\mathrm{p}}=0.719 \pm 0.005$ and $L_{\mathrm{s}} / L_{\mathrm{p}}=0.403 \pm 0.015$ (both for the $V$ band).

\subsubsection{Abundances for AD Boo}

Two high $S$ / $N$ FEROS spectra are available for abundance analyses of AD Boo; see Table 1. We have concentrated on the one with the highest $S / N$, which gives significantly more accurate abundances. Due to the relatively high rotational velocities and low luminosity ratio, the number of suitable unblended lines is far lower than for VZ Hya and WZ Oph, especially for the secondary component. ATLAS 9 models were used, and the effective temperatures, surface gravities and rotational velocities listed in Table 21 were adopted, together with microturbulence velocities of $1.5 \mathrm{~km} \mathrm{~s}^{-1}$ for both components. The temperatures, which are derived from the uvby indices of the components, could not be constrained further from the spectroscopic analysis.

As seen in Table 10, the $[\mathrm{Fe} / \mathrm{H}]$ values from the $\mathrm{Fe} I$ lines (both components) and the few Fe II lines (primary) agree well, and identical results are obtained if MARCS models are used. Changing model temperatures by the uncertainty of the effective temperatures $( \pm 120 \mathrm{~K})$ modifies $[\mathrm{Fe} / \mathrm{H}]$ from the Fe I lines by about \pm 0.10 dex, whereas the Fe II result is changed by only \pm 0.04 dex. If $0.5 \mathrm{~km} \mathrm{~s}^{-1}$ higher microturbulence velocities are adopted, $[\mathrm{Fe} / \mathrm{H}]$ decreases by about 0.10 dex for both neutral and ionized lines. Taking these contributions into account, we obtain an average metallicity of $[\mathrm{Fe} / \mathrm{H}]=+0.07 \pm 0.14$ for $\mathrm{AD} \mathrm{Boo.}$ Abundances for $\mathrm{Ca}$ and $\mathrm{Ni}$ are based on very few lines; although higher than for $\mathrm{Fe}$, they agree within the uncertainties.

Metallicities have also been derived by comparing the disentangled CfA spectra to the extensive library of synthetic 
Table 9. Adopted photometric elements for AD Boo. The individual flux and luminosity ratios are based on the mean stellar and orbital parameters.

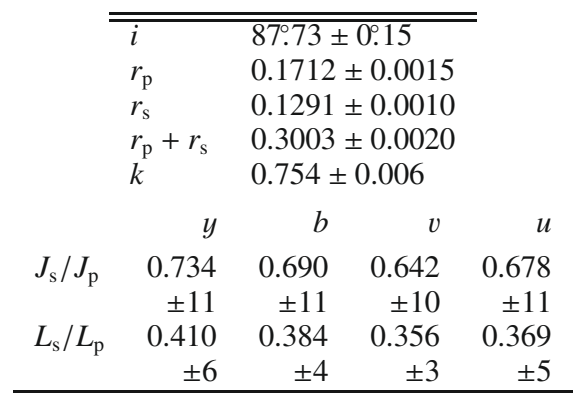

Table 10. Abundances $([\mathrm{El} . / \mathrm{H}])$ for the primary and secondary components of AD Boo. $N$ is the number of lines used per ion.

\begin{tabular}{lllrllr}
\hline \hline & \multicolumn{3}{c}{ Primary } & \multicolumn{3}{c}{ Secondary } \\
Ion & {$[\mathrm{El} . / \mathrm{H}]$} & rms & $N$ & {$[\mathrm{El} . / \mathrm{H}]$} & rms & $N$ \\
\hline Ca I & +0.22 & 0.06 & 4 & +0.20 & 0.23 & 3 \\
Fe I & +0.08 & 0.15 & 33 & +0.06 & 0.17 & 14 \\
Fe II & +0.09 & 0.21 & 4 & & & \\
Ni I & +0.22 & 0.11 & 3 & +0.16 & & 1 \\
\hline
\end{tabular}

spectra especially prepared for the $45 \AA$ region centered at $5187 \AA$. Surface gravities were fixed as above, and synchronous rotation of the components was adopted. The best fits are obtained for $[\mathrm{M} / \mathrm{H}]=+0.13$ and $T_{\mathrm{eff}}=6450 \mathrm{~K}$ (primary), and $[\mathrm{M} / \mathrm{H}]=+0.30$ and $T_{\text {eff }}=6200 \mathrm{~K}$ (secondary), but a strong correlation between metallicities and temperatures exists, and the determinations are therefore quite uncertain. We note that the temperature difference between the components becomes smaller than established from the light curve analyses. Forcing the metallicities of the components to be identical, we derive $[\mathrm{M} / \mathrm{H}]=+0.25$ and temperatures of 6530 and $6160 \mathrm{~K}$ for the components, in good agreement with the photometric temperatures. Estimated uncertainties of metallicities and temperatures are (at least) 0.1 dex and $100 \mathrm{~K}$.

Grids of TODCOR analyses of the CfA spectra for a range of adopted template metallicities yield a metallicity estimate of $[\mathrm{M} / \mathrm{H}]=+0.33$, assuming temperatures of 6575 and $6145 \mathrm{~K}$, and forcing the results for the components to be identical.

Finally, the calibrations by Holmberg et al. (2007) and the de-reddened $u v b y$ indices (Table 21 ) yield $[\mathrm{Fe} / \mathrm{H}]=+0.28 \pm 0.15$ for the primary component ("blue" calibration) and $[\mathrm{Fe} / \mathrm{H}]=$ $+0.10 \pm 0.13$ for the secondary.

Within the fairly large errors, the results from the different methods are in reasonable agreement. Giving higher weight to the analysis of the FEROS spectra, we adopt $[\mathrm{Fe} / \mathrm{H}]=+0.10 \pm$ 0.15 for AD Boo. This result should, however, be checked and preferably improved via analyses of disentangled component spectra based on a sufficiently large number of high-dispersion spectra.

\subsection{VZHya}

VZ Hya $=$ HD $72257=$ HIP $41834(P=2.90)$ was discovered by O'Connell (1932) to be an eclipsing binary, and spectroscopic elements were obtained from $75 \AA / \mathrm{mm}$ spectra by Struve (1945). The currently most reliable absolute dimensions of the components of VZ Hya are those given by Popper (1980) in his critical review on stellar masses.

\subsubsection{Radial velocities and spectroscopic orbit for VZHya}

Our TODCOR analysis of VZ Hya was based on 42 usable CfA spectra outside of eclipse, observed from January 1989 to April 1991. Templates of solar metallicity, $\log g=4.5$ (both components), and temperatures of $6750 \mathrm{~K}$ (primary) and $6250 \mathrm{~K}$ (secondary) were used to derive the radial velocities. As seen in Fig. 8, TODCOR corrections for systematics in the radial velocities are fairly small, typically under $1 \mathrm{~km} \mathrm{~s}^{-1}$ for both components. They make very little difference in the masses, changing them by only $+0.4 \%$ (primary) and $-0.1 \%$ (secondary), which is well within the errors. The scatter around the orbit is about the same with and without corrections applied. Interpolating between $\log g=4.0$ and 4.5 grids to the exact gravities given in Table 21 , we obtain temperatures of $6590 \pm 100 \mathrm{~K}$ for the primary and $6150 \pm 150 \mathrm{~K}$ for the secondary, both in excellent agreement with the photometric values; see Sect. 4 . The corresponding light ratio is $\ell_{\mathrm{s}} / \ell_{\mathrm{p}}=0.53 \pm 0.02$ at the mean wavelength of our observations ( $5187 \AA$ ). Finally, the grids of cross-correlations give best $v \sin i$ values of 21 and $20 \mathrm{~km} \mathrm{~s}^{-1}$, with errors of $2 \mathrm{~km} \mathrm{~s}^{-1}$ and $3 \mathrm{~km} \mathrm{~s}^{-1}$, respectively; they are consistent with synchronous rotation.

The spectroscopic orbital solution for VZHya is given in Table 11, and the observations and computed orbit are shown graphically in Fig. 9 along with the residuals for each star. Our velocity semiamplitudes agree well with those by Popper (1965), $\left(K_{\mathrm{p}}, K_{\mathrm{s}}\right)=(94.3 \pm 2.3,103.9 \pm 1.8)$ but are significantly more accurate.

For comparison, we have also disentangled the 29 best CfA spectra outside of eclipse and obtain velocity semiamplitudes of $K_{\mathrm{p}}=94.62$ and $K_{\mathrm{s}}=105.87$, in good agreement with the results based on TODCOR velocities.

\subsubsection{Photometric elements for VZHya}

The $u v b y$ light curves of VZ Hya contain 1180 observations in each band and were observed on 44 nights during four periods between February 1989 and April 1992 (CVG08). The average observational accuracy per point is about $4 \mathrm{mmag}(v b y)$ and $6 \mathrm{mmag}(u)$, and throughout all phases the points scatter at these levels, indicating that the components of VZHya are constant within the precision of our data. Furthermore, we see no signs of emission in $\mathrm{Ca}$ II $\mathrm{H}$ and $\mathrm{K}$, or in $\mathrm{H}_{\alpha}$, in the FEROS spectra.

EBOP and WD solutions based on linear limb-darkening coefficients by van Hamme (1993) are shown in Tables 12 and 13, respectively. As seen, the results from the different bands agree very well, and the two models lead to practically identical photometric elements. The same holds for WINK solutions not included here. If we adopt instead linear limb-darkening coefficients by Claret (2000), which are $0.05-0.08$ higher, the orbital inclination decreases by 0.2 , and the relative radius is unchanged for the primary component but increases by 0.0009 for the secondary. If adjusted, the linear limb-darkening coefficients by van Hamme are reproduced to within \pm 0.05 and the solutions are close to the results in Tables 12 and 13. WD solutions including non-linear limb darkening (see Sect. 2.4) give photometric elements very close to those presented in Table 13. O-C residuals of the $b$ observations from the theoretical light curve computed for the photometric elements given in Table 12 are shown in Fig. 10.

As done for AD Boo, in order to assign realistic errors to the photometric elements, we have supplemented these analyses with 10000 JKTEBOP Monte Carlo simulations in each band, and EBOP solutions for a range of fixed $k$ values near 0.85 ; see Figs. 11 and 12. The adopted photometric elements listed 

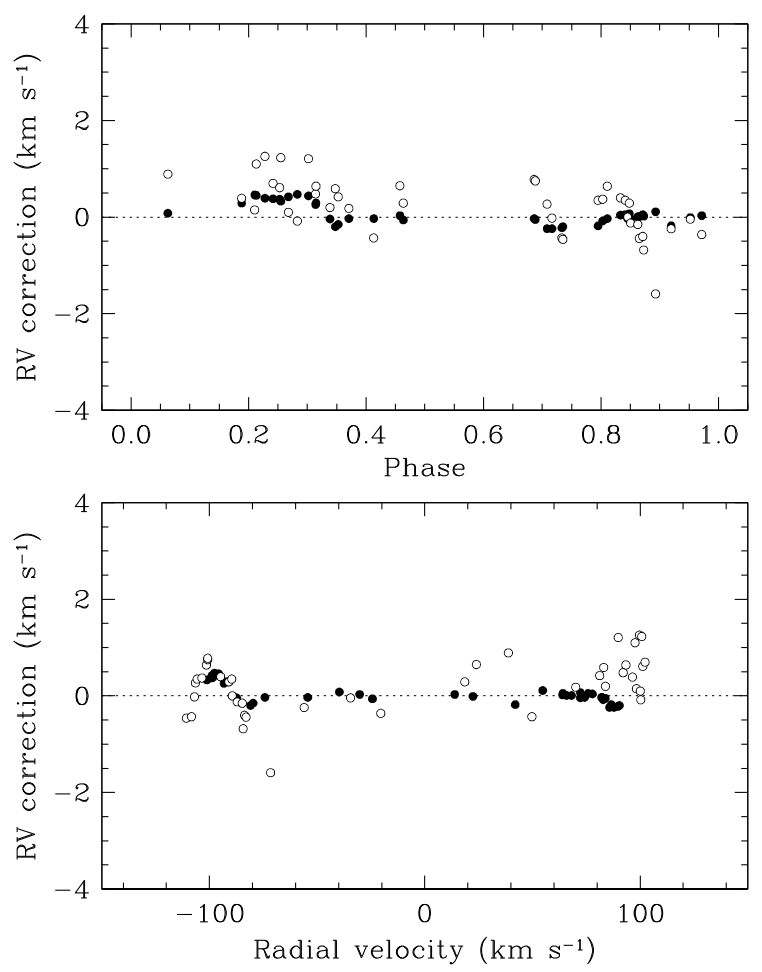

Fig. 8. Systematic errors in the raw TODCOR velocities of VZHya, determined from simulations with synthetic binary spectra (filled circles: primary; open circles: secondary). The differences are plotted both as function of orbital phase (upper panel) and radial velocity (lower panel), and have been applied to the measured velocities as corrections. Phase 0.0 corresponds to central primary eclipse.

Table 11. Spectroscopic orbital solution for VZ Hya. $T$ is the time of central primary eclipse.

\begin{tabular}{lr}
\hline \hline Parameter & \multicolumn{1}{c}{ Value } \\
\hline Adjusted quantities: & \\
$K_{\mathrm{p}}\left(\mathrm{km} \mathrm{s}^{-1}\right)$ & $94.92 \pm 0.19$ \\
$K_{\mathrm{s}}\left(\mathrm{km} \mathrm{s}^{-1}\right)$ & $105.31 \pm 0.34$ \\
$\gamma\left(\mathrm{km} \mathrm{s}^{-1}\right)$ & $-4.57 \pm 0.14$ \\
Adopted quantities: & \\
$P($ days $)$ & 2.90430023 \\
$T$ (HJD-2 400 000) & 48273.63450 \\
$e$ & 0.00 \\
Derived quantities: & \\
$M_{\mathrm{p}} \sin ^{3} i\left(M_{\odot}\right)$ & $1.2705 \pm 0.0090$ \\
$M_{\mathrm{s}} \sin ^{3} i\left(M_{\odot}\right)$ & $1.1451 \pm 0.0061$ \\
$a_{\mathrm{p}} \sin i\left(10^{6} \mathrm{~km}\right)$ & $3.7907 \pm 0.0079$ \\
$a_{\mathrm{s}} \sin i\left(10^{6} \mathrm{~km}\right)$ & $4.2057 \pm 0.0140$ \\
$a \sin i\left(R_{\odot}\right)$ & $11.489 \pm 0.023$ \\
Other quantities pertaining to the fit: & \\
$N_{\text {obs }}$ & 42 \\
Time span (days) & 1.04 \\
$\sigma_{\mathrm{p}}\left(\mathrm{km} \mathrm{s}^{-1}\right)$ & 1.85 \\
$\sigma_{\mathrm{s}}\left(\mathrm{km} \mathrm{s}^{-1}\right)$ & \\
\hline
\end{tabular}

in Table 14 are the weighted mean values of the EBOP solutions adopting the linear limb-darkening coefficients by van Hamme. Errors are based on the Monte Carlo simulations and a comparison between the $u v b y$ solutions. Our results are significantly more accurate than obtained in previous studies of VZ Hya. At phase 0.0 , about $76 \%$ of the $y$ light from the primary component is eclipsed, and at phase 0.5 about $98 \%$ of the $y$ light of the

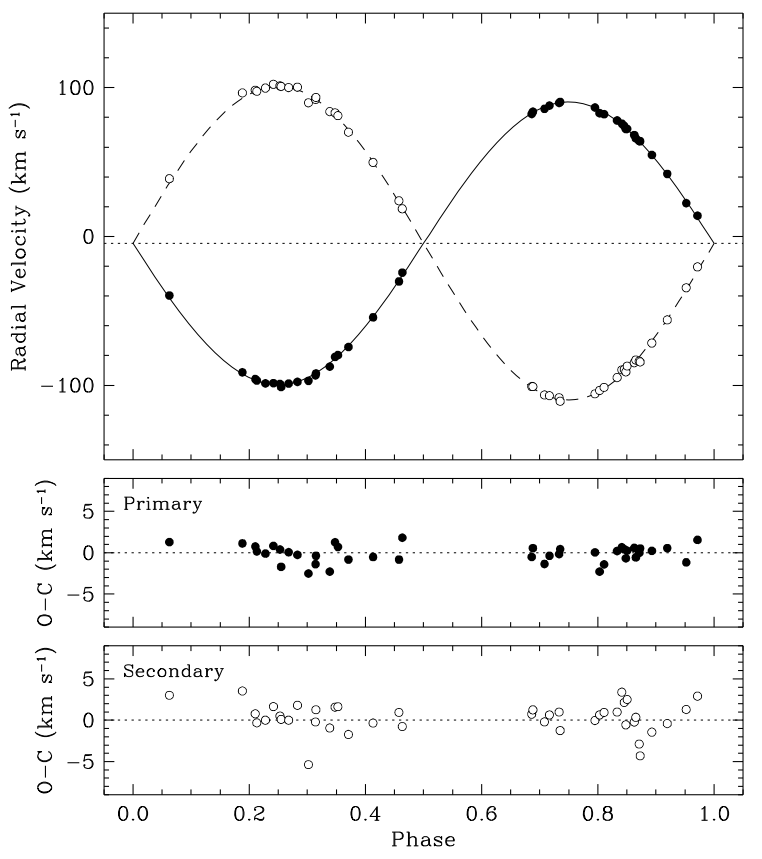

Fig. 9. Spectroscopic orbital solution for VZ Hya (solid line: primary; dashed line: secondary) and radial velocities (filled circles: primary; open circles: secondary). The dotted line (upper panel) represents the center-of-mass velocity of the system. Phase 0.0 corresponds to central primary eclipse.

Table 12. Photometric solutions for VZ Hya from the EBOP code adopting linear limb darkening coefficients by van Hamme (1993). The errors quoted for the free parameters are the formal errors determined from the iterative least squares solution procedure.

\begin{tabular}{lrrrr}
\hline \hline & $y$ & $b$ & $v$ & $u$ \\
\hline$i\left(^{\circ}\right)$ & 88.83 & 88.91 & 88.88 & 88.89 \\
& \pm 6 & \pm 5 & \pm 5 & \pm 8 \\
$r_{\mathrm{p}}$ & 0.1142 & 0.1144 & 0.1144 & 0.1140 \\
& \pm 4 & \pm 3 & \pm 3 & \pm 5 \\
$r_{\mathrm{s}}$ & 0.0969 & 0.0967 & 0.0969 & 0.0970 \\
$k$ & 0.849 & 0.845 & 0.847 & 0.850 \\
& \pm 7 & \pm 4 & \pm 5 & \pm 8 \\
$r_{\mathrm{p}}+r_{\mathrm{s}}$ & 0.2111 & 0.2111 & 0.2113 & 0.2110 \\
$u_{\mathrm{p}}$ & 0.52 & 0.60 & 0.67 & 0.64 \\
$u_{\mathrm{s}}$ & 0.54 & 0.63 & 0.71 & 0.71 \\
$y_{\mathrm{p}}$ & 0.34 & 0.39 & 0.44 & 0.52 \\
$y_{\mathrm{s}}$ & 0.35 & 0.41 & 0.47 & 0.55 \\
$J_{\mathrm{s}} / J_{\mathrm{p}}$ & 0.7983 & 0.7665 & 0.7306 & 0.7649 \\
& \pm 20 & \pm 16 & \pm 18 & \pm 29 \\
$L_{\mathrm{s}} / L_{\mathrm{p}}$ & 0.5701 & 0.5402 & 0.5143 & 0.5364 \\
$\sigma$ (mag.) & 0.0046 & 0.0040 & 0.0044 & 0.0068 \\
\hline
\end{tabular}

secondary is blocked out, meaning that secondary eclipse is almost total. The spectroscopic light ratio of $\ell_{\mathrm{s}} / \ell_{\mathrm{p}}=0.53 \pm 0.02$ at $5187 \AA$ agrees quite well with that from the light curve solutions (0.56).

Previous analyses based on the photoelectric $U B V$ light curves by Walker (1970) gave 3-4\% larger relative radii for the primary component, whereas those for the secondary component agree well with our result (Walker 1970; Wood 1971, Cester et al. 1978). Another set of photoelectric $U B V$ light curves was published by Padalia \& Srivastava (1975), who found primary eclipse to be an occultation (larger star in front) rather than a transit (smaller star in front). This picture of VZ Hya was soon 
Table 13. Photometric solutions for VZ Hya from the WD code adopting linear limb darkening coefficients by van Hamme (1993); see Table 12. Gravity darkening exponents of 0.33 and bolometric albedo coefficients of 0.5 were adopted, as appropriate for convective envelopes. $T_{\text {eff,p }}$ was assumed to be $6650 \mathrm{~K}$.

\begin{tabular}{|c|c|c|c|c|}
\hline & $y$ & $b$ & $v$ & $u$ \\
\hline \multirow[t]{2}{*}{$i\left(^{\circ}\right)$} & 88.90 & 88.92 & 88.95 & 88.98 \\
\hline & \pm 2 & \pm 1 & \pm 2 & \pm 2 \\
\hline \multirow[t]{2}{*}{$\Omega_{\mathrm{p}}$} & 9.649 & 9.666 & 9.639 & 9.668 \\
\hline & \pm 8 & \pm 7 & \pm 8 & \pm 11 \\
\hline \multirow[t]{2}{*}{$\Omega_{\mathrm{s}}$} & 10.349 & 10.329 & 10.355 & 10.343 \\
\hline & \pm 8 & \pm 7 & \pm 8 & \pm 11 \\
\hline$r_{\mathrm{p}}$ & 0.1144 & 0.1142 & 0.1146 & 0.1142 \\
\hline$r_{\mathrm{s}}$ & 0.0969 & 0.0971 & 0.0968 & 0.0969 \\
\hline$k$ & 0.847 & 0.850 & 0.845 & 0.849 \\
\hline$r_{\mathrm{p}}+r_{\mathrm{s}}$ & 0.2113 & 0.2113 & 0.2114 & 0.2111 \\
\hline \multirow[t]{2}{*}{$T_{\text {eff }, s}$} & 6272 & 6252 & 6229 & 6182 \\
\hline & \pm 2 & \pm 1 & \pm 2 & \pm 3 \\
\hline$L_{\mathrm{s}} / L_{\mathrm{p}}$ & 0.5686 & 0.5464 & 0.5125 & 0.5347 \\
\hline$\sigma$ (mag.) & 0.0046 & 0.0039 & 0.0043 & 0.0068 \\
\hline
\end{tabular}
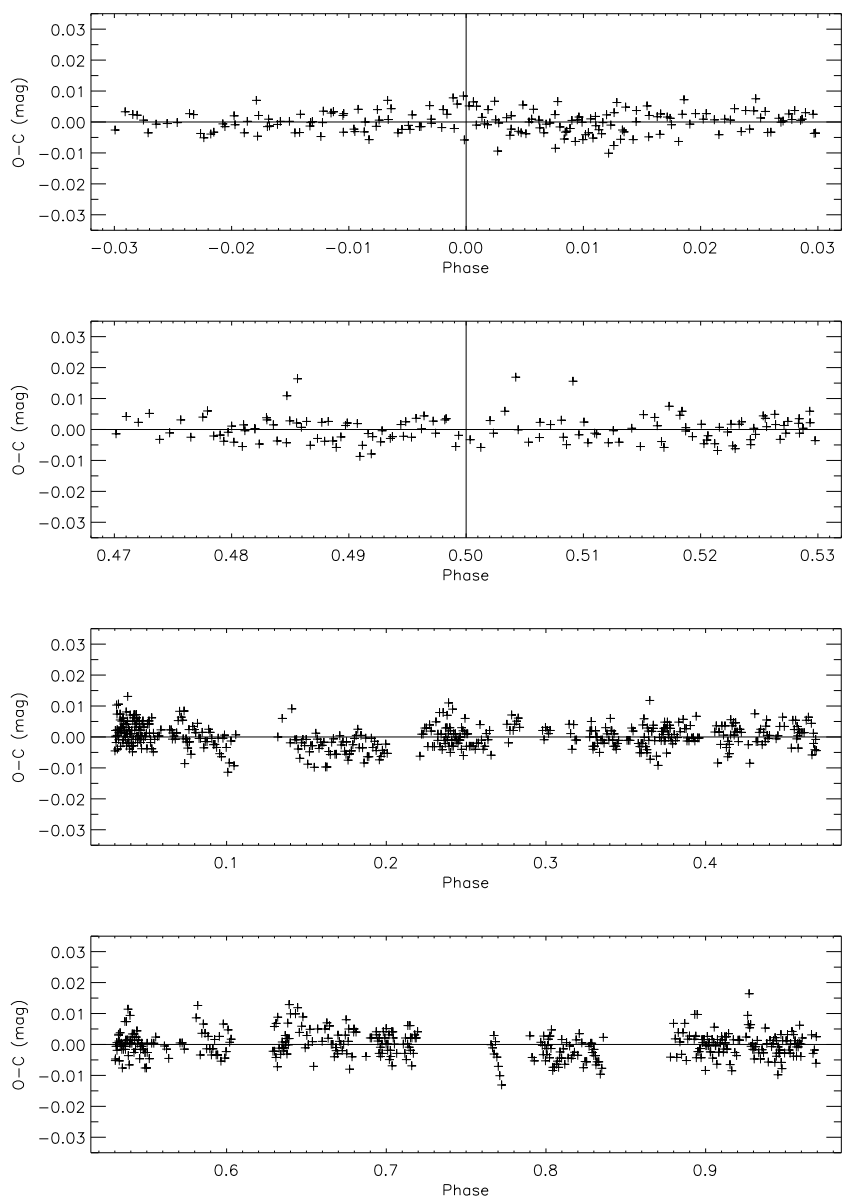

Fig. 10. (O-C) residuals of the VZHya $b$-band observations from the theoretical light curve computed for the photometric elements given in Table 12 .

shown by Popper (1976) to be wrong but was nevertheless defended once more by Padalia (1986).

\subsubsection{Abundances for VZHya}

Five FEROS spectra are available for the detailed abundance analysis of VZHya; see Table 1. As mentioned in Sect. 2.6,
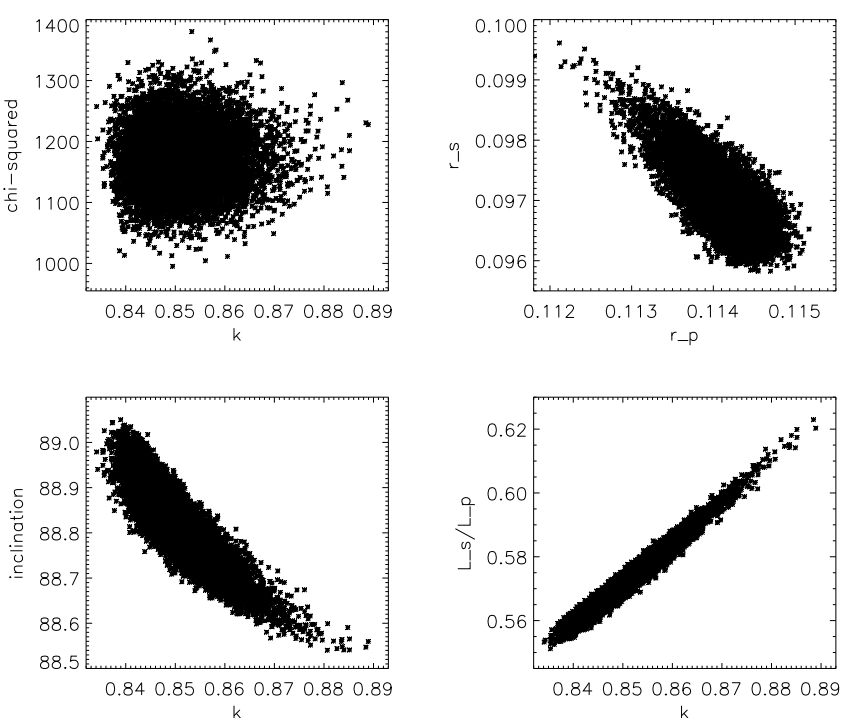

Fig. 11. Best fitting parameter values for the 10000 synthetic VZ Hya $y$ light curves created for the Monte Carlo analysis.
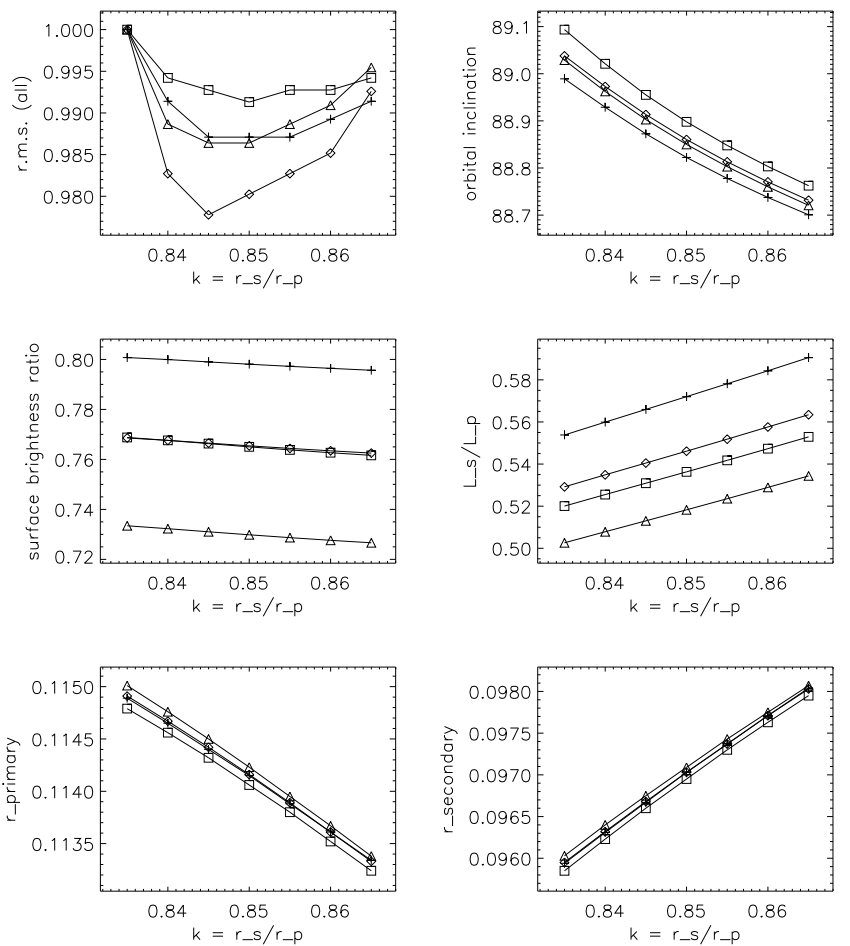

Fig. 12. EBOP solutions for VZ Hya for a range of fixed $k$ values. Linear limb-darkening coefficients by van Hamme (1993) were adopted. The upper left panel shows normalized rms errors of the fit to the observations. Symbols are: cross $y$; diamond $b$; triangle $v$; square $u$.

disentangling of so few spectra was unsuccessful, and we have instead used the three most suitable observed spectra and analysed them as double-lined. The results from the VWA analysis are presented in Table 15. ATLAS9 models were used, and the final effective temperatures, surface gravities and rotational velocities listed in Table 21 were adopted, together with microturbulence velocities of 2.0 and $1.6 \mathrm{~km} \mathrm{~s}^{-1}$, respectively, calculated from the calibration by Edvardsson et al. (1993). For this choice of parameters there is no dependence on equivalent width and excitation potential for abundances determined from individual 
Table 14. Adopted photometric elements for VZHya. The individual flux and luminosity ratios are based on the mean stellar and orbital parameters.

\begin{tabular}{lrrrr} 
& & & \\
& \multicolumn{1}{c}{$88^{\circ} .88 \pm 0.09$} & \\
& $r_{\mathrm{p}}$ & $0.1143 \pm 0.0004$ & \\
& $r_{\mathrm{s}}$ & $0.0968 \pm 0.0006$ & \\
& $r_{\mathrm{p}}+r_{\mathrm{s}}$ & $0.2111 \pm 0.0004$ & \\
& $k$ & & $0.847 \pm 0.007$ & \\
& $y$ & $b$ & $v$ & $u$ \\
$J_{\mathrm{s}} / J_{\mathrm{p}}$ & 0.799 & 0.766 & 0.730 & 0.764 \\
& \pm 12 & \pm 12 & \pm 11 & \pm 12 \\
$L_{\mathrm{s}} / L_{\mathrm{p}}$ & 0.569 & 0.543 & 0.515 & 0.532 \\
& \pm 9 & \pm 6 & \pm 6 & \pm 9 \\
\hline
\end{tabular}

Table 15. Abundances $([\mathrm{El} . / \mathrm{H}])$ for the primary and secondary components of VZ Hya. $N$ is the number of lines used per ion.

\begin{tabular}{llrrrrr}
\hline \hline & \multicolumn{3}{c}{ Primary } & \multicolumn{3}{c}{ Secondary } \\
Ion & {$[\mathrm{El} . / \mathrm{H}]$} & rms & $N$ & {$[\mathrm{El} . / \mathrm{H}]$} & rms & $N$ \\
\hline Si I & -0.14 & 0.17 & 7 & -0.18 & 0.16 & 12 \\
Ca I & -0.13 & 0.15 & 8 & -0.17 & 0.16 & 7 \\
Sc II & -0.36 & 0.14 & 2 & -0.33 & 0.07 & 3 \\
Ti I & -0.25 & & 1 & -0.21 & 0.17 & 4 \\
Ti II & -0.48 & 0.43 & 3 & -0.30 & 0.09 & 2 \\
Cr I & -0.23 & 0.16 & 6 & -0.24 & 0.11 & 4 \\
Cr II & -0.29 & 0.03 & 4 & -0.43 & 0.06 & 4 \\
Fe I & -0.21 & 0.16 & 91 & -0.19 & 0.18 & 69 \\
Fe II & -0.32 & 0.18 & 12 & -0.14 & 0.26 & 11 \\
Ni I & -0.20 & 0.23 & 9 & -0.07 & 0.27 & 16 \\
\hline
\end{tabular}

lines, which on the other hand appears if temperatures are changed by about $150 \mathrm{~K}$.

As seen, the $[\mathrm{Fe} / \mathrm{H}]$ values from the many $\mathrm{Fe}$ I lines agree well, whereas those from the much fewer Fe II lines are lower (primary) and higher (secondary). Similar results are obtained if MARCS models are used, and we have not been able to identify the cause of the discrepancies. Changing model temperatures by the uncertainty of the effective temperatures $( \pm 150 \mathrm{~K})$ modifies $[\mathrm{Fe} / \mathrm{H}]$ from the $\mathrm{Fe} I$ lines by $\pm 0.10 \mathrm{dex}$, whereas the Fe II results are changed by only \pm 0.04 dex. If $0.5 \mathrm{~km} \mathrm{~s}^{-1}$ higher microturbulence velocities are adopted, $[\mathrm{Fe} / \mathrm{H}]$ decreases by about 0.06 dex for both neutral and ionized lines. Taking these contributions into account, we obtain a weighted average of $[\mathrm{Fe} / \mathrm{H}]=-0.20 \pm 0.12$ for VZ Hya.

Excluding ions with few measurements, we find that within the uncertainties, identical abundances are derived from $\mathrm{Cr}$ I and $\mathrm{Ni}$ I lines. The $\alpha$-elements $\mathrm{Si}$ and Ca seem slightly enhanced, but the difference is barely significant.

For the metallicities derived by comparing the disentangled CfA spectra with the library of synthetic spectra, surface gravities were fixed as above, and synchronous rotation of the components was adopted. The best fits are obtained for $[\mathrm{M} / \mathrm{H}]=+0.09$ and $T_{\text {eff }}=6630 \mathrm{~K}$ (primary), and $[\mathrm{M} / \mathrm{H}]=-0.23$ and $T_{\text {eff }}=$ $6130 \mathrm{~K}$ (secondary), but as for AD Boo, a strong correlation between metallicities and temperatures exist, and the determinations are therefore quite uncertain. Forcing the metallicities of the components to be identical, we derive $[\mathrm{M} / \mathrm{H}]=-0.15$, and temperatures of 6595 and $6185 \mathrm{~K}$ for the components, in good agreement with the FEROS result and the photometric temperatures. Estimated uncertainties of these CfA metallicities and temperatures are (at least) $0.1 \mathrm{dex}$ and $100 \mathrm{~K}$, respectively.

Comparing TODCOR analyses of the observed CfA spectra for a range of adopted template metallicities yields a metallicity estimate of $[\mathrm{M} / \mathrm{H}]=+0.02$, assuming temperatures of 6650 and $6300 \mathrm{~K}$ and forcing the metal abundance of the components to be identical.

Finally, the calibrations by Holmberg et al. (2007) and the de-reddened uvby indices in Table 21 yield $[\mathrm{Fe} / \mathrm{H}]=$ $+0.05 \pm 0.15$ for the primary component ("blue" calibration) and $[\mathrm{Fe} / \mathrm{H}]=-0.08 \pm 0.12$ for the secondary, marginally in agreement with the FEROS result, which we adopt for VZHya.

\section{3. $W Z O p h$}

WZ Oph $=$ HD $154676=\operatorname{HIP} 83719(P=4.18)$ was discovered by Metcalf to be an eclipsing binary (Pickering 1917), and Sanford (1937) obtained the first radial velocities. The currently best modern analysis of WZ Oph, leading to masses and radii accurate to $3-4 \%$, was done by Popper (1965) from photoelectric light curves and $20 \AA / \mathrm{mm}$ spectra.

For the spectroscopic and photometric analyses of WZ Oph we have adopted the well established ephemeris from CVG08, where the zero point has been shifted by half a period compared to that by Popper (1965). We note that the primary component eclipsed at phase 0.0 according to our adopted ephemeris, and corresponding to the slightly deeper minimum for the $u v b y$ light curves, is given by Popper (1965) as component 2. Popper found that his component 2 , i.e. our primary component, has slightly stronger spectral lines. This is consistent with our measurements of equivalent widths in the disentangled FEROS spectra for lines stronger than about $100 \mathrm{~m} \AA$.

\subsubsection{Radial velocities and spectroscopic orbit for WZOph}

Radial velocities for the components of WZOph were determined with TODCOR on the basis of 40 usable CfA spectra outside of eclipse, observed from February 1989 to September 1991. Identical templates of solar composition with $\log g=4.0$ and a temperature of $6250 \mathrm{~K}$ were adopted for the two stars. The TODCOR corrections for systematics shown in Fig. 13 are less than $0.5 \mathrm{~km} \mathrm{~s}^{-1}$ at all phases for both components. This makes a difference of only $+0.3 \%$ in the minimum masses, about half their uncertainties, and the scatter of the orbit does not change much with corrections. $v \sin i$ values of 18 and $17 \mathrm{~km} \mathrm{~s}^{-1}$ are obtained for the primary and secondary components, respectively, with estimated uncertainties of $1 \mathrm{~km} \mathrm{~s}^{-1}$. They are consistent with synchronous rotation.

Our best spectroscopic estimates of the effective temperatures of the components by interpolating to a $\log g$ value close to that listed in Table 21 are $6260 \mathrm{~K}$ for the primary and $6340 \mathrm{~K}$ for the secondary, assuming solar metallicity. Interpolating to the metallicity of $[\mathrm{M} / \mathrm{H}]=-0.27$ (see below) gives $6050 \mathrm{~K}$ and $6120 \mathrm{~K}$. The lower values for the primary compared to the secondary disagree formally with the trend from photometry, which gives a slightly hotter primary based on the slightly deeper primary eclipse. However, the difference is well within the spectroscopic error estimate of $150 \mathrm{~K}$. The light ratio is $\ell_{\mathrm{s}} / \ell_{\mathrm{p}}=0.98 \pm 0.02$ at the mean wavelength of our observations (5187 ̊).

The spectroscopic orbital solution for WZOph is given in Table 16 and the observations and computed orbit are shown graphically in Fig. 14 along with the residuals for each star. For comparison, we have also disentangled the 40 CfA spectra outside of eclipse and obtain velocity semiamplitudes of $K_{\mathrm{p}}=88.62$ and $K_{\mathrm{s}}=88.92$, in good agreement with the results based on the TODCOR velocities. 

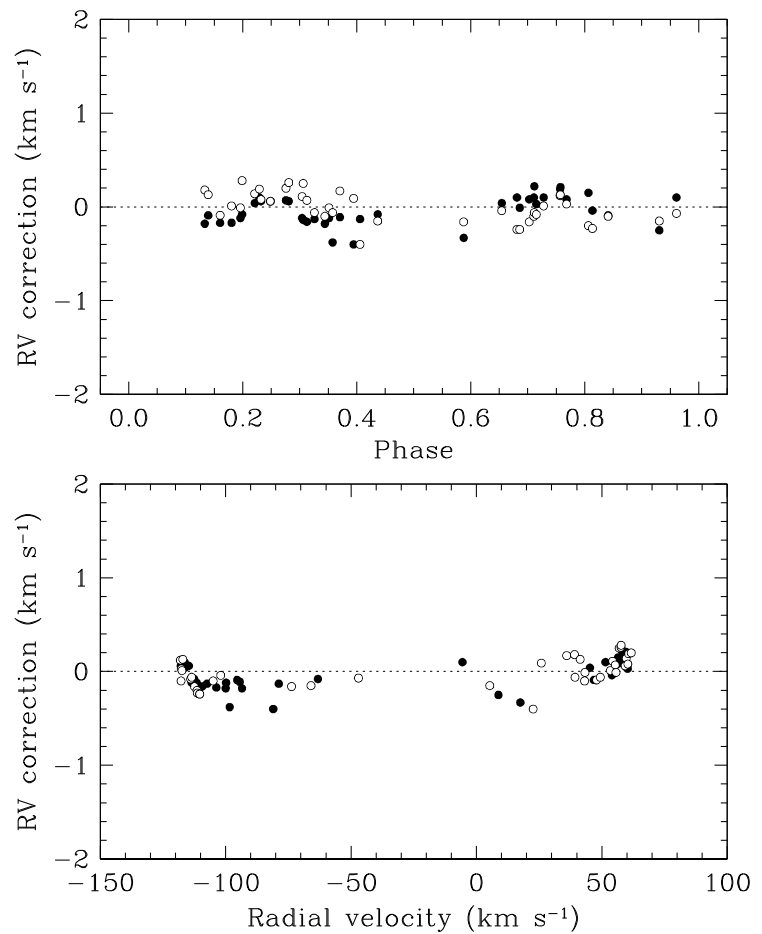

Fig. 13. Systematic errors in the raw TODCOR velocities of WZOph, determined from simulations with synthetic binary spectra (filled circles: primary; open circles: secondary). The differences are plotted both as function of orbital phase (upper panel) and radial velocity (lower panel), and have been applied to the measured velocities as corrections. Phase 0.0 corresponds to central primary eclipse.

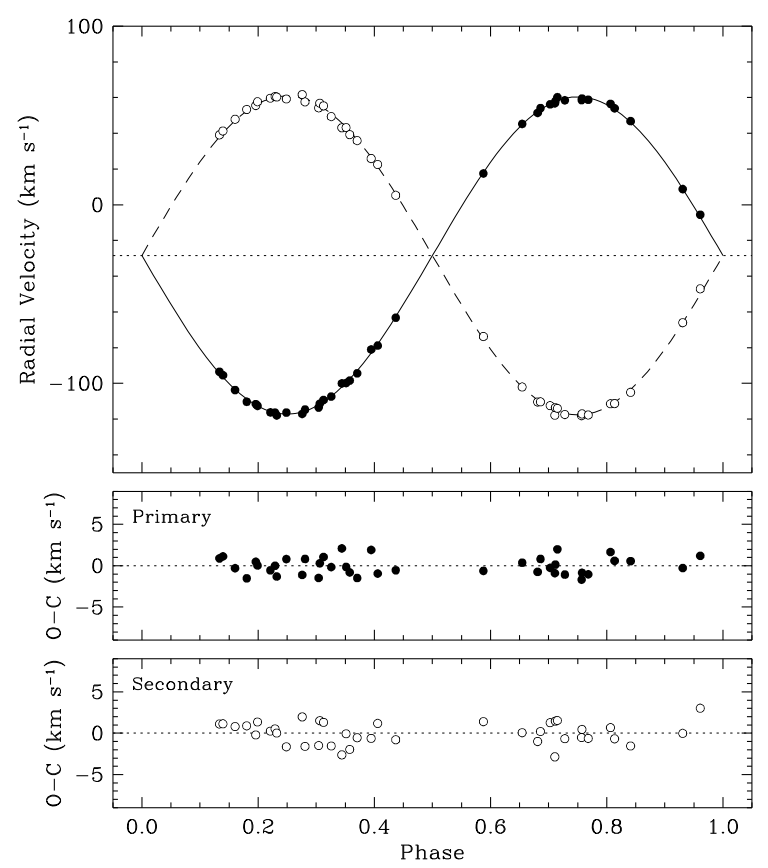

Fig. 14. Spectroscopic orbital solution for WZ Oph (solid line: primary; dashed line: secondary) and radial velocities (filled circles: primary; open circles: secondary). The dotted line (upper panel) represents the center-of-mass velocity of the system. Phase 0.0 corresponds to central primary eclipse.

Our velocity semiamplitudes are larger than those by Popper $(1965),\left(K_{\mathrm{p}}, K_{\mathrm{s}}\right)=(85.9 \pm 1.8,87.0 \pm 2.0)$, and as in the case of VZ Hya they are of much higher precision.
Table 16. Spectroscopic orbital solution for WZOph. $T$ is the time of central primary eclipse.

\begin{tabular}{lr}
\hline \hline Parameter & \multicolumn{1}{c}{ Value } \\
\hline Adjusted quantities: & \\
$K_{\mathrm{p}}\left(\mathrm{km} \mathrm{s}^{-1}\right)$ & $88.77 \pm 0.19$ \\
$K_{\mathrm{s}}\left(\mathrm{km} \mathrm{s}^{-1}\right)$ & $89.26 \pm 0.24$ \\
$\gamma\left(\mathrm{km} \mathrm{s}^{-1}\right)$ & $-28.36 \pm 0.13$ \\
Adopted quantities: & \\
$P($ days $)$ & 4.18350681 \\
$T$ (HJD-2 400000$)$ & 50353.78331 \\
$e$ & 0.00 \\
Derived quantities: & \\
$M_{\mathrm{p}} \sin ^{3} i\left(M_{\odot}\right)$ & $1.2262 \pm 0.0073$ \\
$M_{\mathrm{s}} \sin ^{3} i\left(M_{\odot}\right)$ & $1.2194 \pm 0.0063$ \\
$a_{\mathrm{p}} \sin i\left(10^{6} \mathrm{~km}\right)$ & $5.106 \pm 0.011$ \\
$a_{\mathrm{s}} \sin i\left(10^{6} \mathrm{~km}\right)$ & $5.135 \pm 0.014$ \\
$a \sin i\left(R_{\odot}\right)$ & $14.714 \pm 0.026$ \\
Other quantities pertaining to the fit: & \\
$N_{\mathrm{obs}}$ & 40 \\
Time span (days) & 918 \\
$\sigma_{\mathrm{p}}\left(\mathrm{km} \mathrm{s}^{-1}\right)$ & 1.04 \\
$\sigma_{\mathrm{s}}\left(\mathrm{km} \mathrm{s}^{-1}\right)$ & 1.33 \\
\hline
\end{tabular}

Table 17. Photometric solutions for WZOph from the EBOP code adopting linear limb darkening coefficients by van Hamme (1993). The errors quoted for the free parameters are the formal errors determined from the iterative least squares solution procedure.

\begin{tabular}{lrrrr}
\hline \hline & $y$ & $b$ & $v$ & $u$ \\
\hline$i\left(^{\circ}\right)$ & 89.08 & 89.09 & 89.06 & 89.11 \\
& \pm 2 & \pm 2 & \pm 2 & \pm 5 \\
$r_{\mathrm{p}}$ & 0.0956 & 0.0950 & 0.0951 & 0.0949 \\
& \pm 8 & \pm 7 & \pm 7 & \pm 11 \\
$r_{\mathrm{s}}$ & 0.0963 & 0.0963 & 0.0960 & 0.0975 \\
$k$ & 1.008 & 1.014 & 1.009 & 1.027 \\
& \pm 16 & \pm 14 & \pm 14 & \pm 22 \\
$r_{\mathrm{p}}+r_{\mathrm{s}}$ & 0.1919 & 0.1913 & 0.1911 & 0.1924 \\
$u_{\mathrm{p}}=u_{\mathrm{s}}$ & 0.56 & 0.65 & 0.73 & 0.74 \\
$y_{\mathrm{p}}=y_{\mathrm{s}}$ & 0.36 & 0.42 & 0.48 & 0.57 \\
$J_{\mathrm{s}} / J_{\mathrm{p}}$ & 0.9878 & 0.9815 & 0.9831 & 0.9668 \\
& \pm 38 & \pm 41 & \pm 46 & \pm 73 \\
$L_{\mathrm{s}} / L_{\mathrm{p}}$ & 1.0035 & 1.0102 & 1.0001 & 1.0209 \\
$\sigma \quad(\mathrm{mag})$ & 0.0053 & 0.0057 & 0.0065 & 0.0104 \\
\hline
\end{tabular}

\subsubsection{Photometric elements for WZ Oph}

The uvby light curves of WZ Oph contain 697 observations in each band and were observed on 41 nights during six periods between March 1991 and March 1997 (CVG08). The average observational accuracy per point is about 4 mmag (vby) and 6 mmag $(u)$, but throughout all phases the points scatter by $1-$ 4 mmag more than this, highest in $u$. At a given phase, the observations from different seasons do not differ systematically, and it is thus unclear if the extra scatter in the light curves is due to slight variability of one or both stars (activity), or perhaps to an unknown instrumental/observational effect not seen in other systems. The Rossby number inferred for the mean component of WZ Oph does not clearly place it in the group of stars that show variability due to spots (Hall 1994), and is thus inconclusive. On the other hand, the high $S / N$ FEROS spectrum taken at phase 0.33 (see Table 1) indicates very weak emission from both components in the $\mathrm{Ca}$ II H and $\mathrm{K}$ lines, but not in $\mathrm{H}_{\alpha}$. This suggests WZ Oph is in fact mildly active. 
Table 18. Photometric solutions for WZ Oph from the WD code adopting linear limb darkening coefficients by van Hamme (1993); see Table 17. Gravity darkening exponents of 0.33 and bolometric albedo coefficients of 0.5 were adopted, as appropriate for convective envelopes. $T_{\text {eff, } p}$ was assumed to be $6165 \mathrm{~K}$.

\begin{tabular}{|c|c|c|c|c|}
\hline & $\bar{y}$ & $\bar{b}$ & $\bar{v}$ & $u$ \\
\hline \multirow[t]{2}{*}{$i\left(^{\circ}\right)$} & 89.11 & 89.11 & 89.08 & 89.14 \\
\hline & \pm 1 & \pm 1 & \pm 2 & \pm 3 \\
\hline \multirow{2}{*}{$\Omega_{\mathrm{p}}$} & 11.454 & 11.510 & 11.554 & 11.444 \\
\hline & \pm 11 & \pm 15 & \pm 14 & \pm 32 \\
\hline \multirow[t]{2}{*}{$\Omega_{\mathrm{s}}$} & 11.334 & 11.299 & 11.310 & 11.276 \\
\hline & \pm 12 & \pm 14 & \pm 17 & \pm 30 \\
\hline$r_{\mathrm{p}}$ & 0.0957 & 0.0952 & 0.0948 & 0.0958 \\
\hline$r_{\mathrm{s}}$ & 0.0963 & 0.0967 & 0.0966 & 0.0969 \\
\hline$k$ & 1.007 & 1.016 & 1.019 & 1.012 \\
\hline$r_{\mathrm{p}}+r_{\mathrm{s}}$ & 0.1920 & 0.1919 & 0.1914 & 0.1927 \\
\hline \multirow[t]{2}{*}{$T_{\mathrm{eff}, s}$} & 6149 & 6141 & 6141 & 6121 \\
\hline & \pm 2 & \pm 2 & \pm 3 & \pm 5 \\
\hline$L_{\mathrm{s}} / L_{\mathrm{p}}$ & 1.0023 & 1.0126 & 1.0180 & 0.9936 \\
\hline$\sigma \quad(\mathrm{mag})$ & 0.0053 & 0.0057 & 0.0065 & 0.0104 \\
\hline
\end{tabular}

EBOP and WD solutions, based on linear limb-darkening coefficients by van Hamme (1993) are presented in Tables 17 and 18, respectively. As seen, the results from the different bands agree well, and the two models lead to nearly identical photometric elements. WINK solutions not included here lead to changes in the relative radii of -0.0008 (primary) and +0.0008 (secondary). Formally, the less massive secondary component is slightly larger and cooler, but more luminous, than the primary for all three models. In reality the two stars are, however, very nearly identical. If we adopt linear limb-darkening coefficients by Claret (2000), which are 0.10 higher than those by van Hamme, the orbital inclination decreases by 0.1 , and the relative radius increases by about 0.0004 for the primary component but is unchanged for the secondary. If adjusted, the $v b y$ linear limbdarkening coefficients by van Hamme are reproduced to within \pm 0.05 and the solutions are close to the results in Table 17. The $u$ coefficients, however, become unrealistically low (0.60), and $i$ changes by $0{ }^{\circ} .2$. WD solutions including non-linear limb darkening (see Sect. 2.4) give photometric elements very close to those presented in Table 18 for the $u v b$ bands, but the $u$ solution now disagrees in having $k \approx 1.05$. $\mathrm{O}-\mathrm{C}$ residuals of the $y$ observations from the theoretical light curve computed for the photometric elements given in Table 17 are shown in Fig. 15.

Realistic errors have been assigned to the photometric elements by performing 10000 JKTEBOP Monte Carlo simulations in each band, and EBOP solutions for a range of fixed $k$ values near 1.0; see Figs. 16 and 17. The adopted photometric elements listed in Table 19 are the weighted mean values of the EBOP solutions adopting the linear limb-darkening coefficients by van Hamme. Errors are based on the Monte Carlo simulations and comparison between the uvby solutions. Adopting the spectroscopic light ratio of $\ell_{\mathrm{s}} / \ell_{\mathrm{p}}=0.98 \pm 0.02$ at $5187 \AA$ would lead to $k=0.996$ (formal error \pm 0.011 ), $r_{\mathrm{p}}=0.0960$, and $r_{\mathrm{s}}=0.0956$. We find that about $92 \%$ of the light of the components is shadowed out at both central eclipses.

For comparison, Popper (1965) obtained slightly smaller relative radii $\left(r_{\mathrm{p}}=0.0945 \pm 0.002, r_{\mathrm{s}}=0.092 \pm 0.001\right)$, and an orbital inclination of $i=89^{\circ} .4 \pm 0.2$ (all uncertainties given by Popper are estimated). From a WINK re-analysis of Popper's photoelectric light curve, Cester et al. (1978) derived $r_{\mathrm{p}}=0.096 \pm 0.001, k=0.971 \pm 0.017$, and $i=89^{\circ} .0 \pm 0.1$.
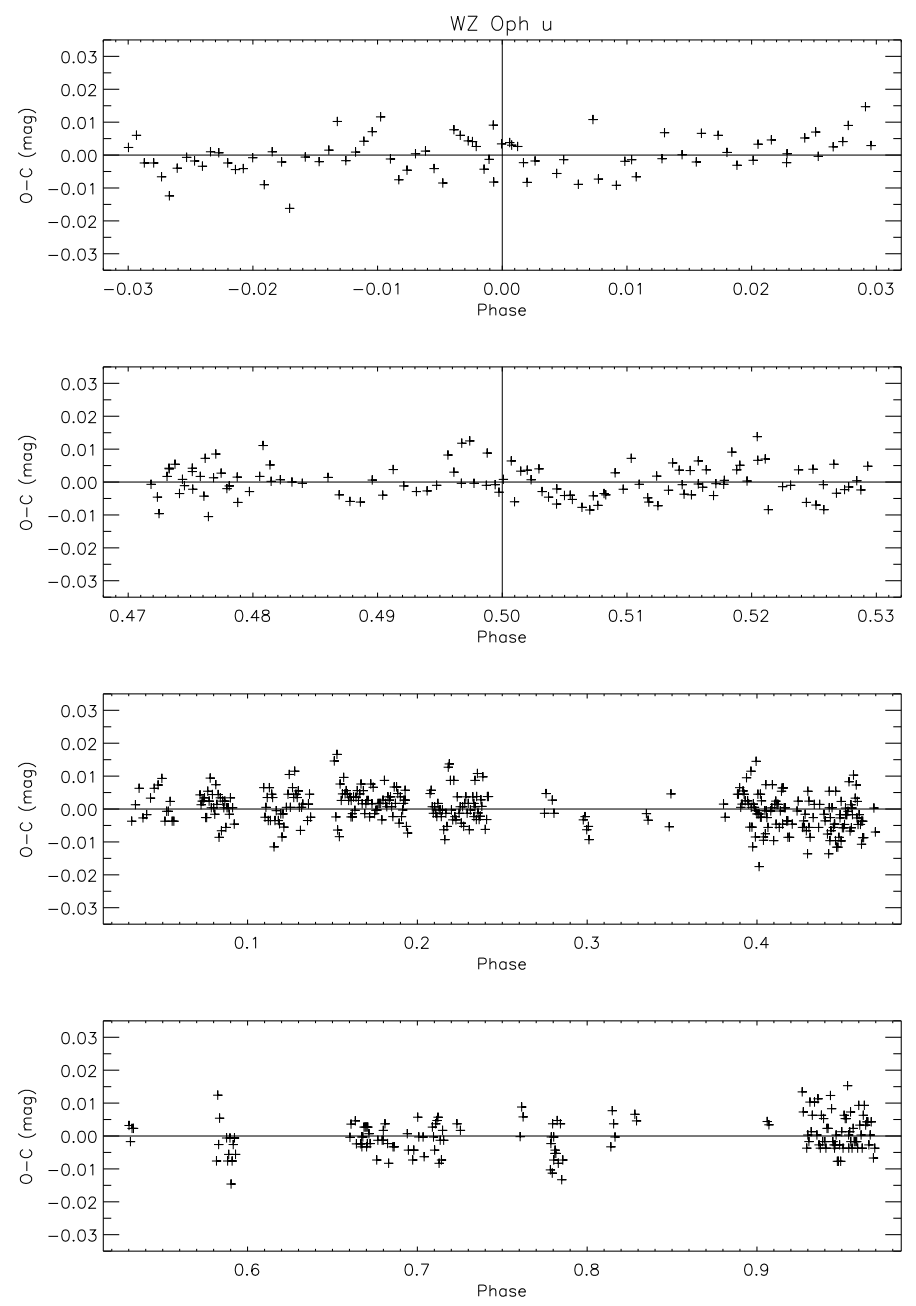

Fig. 15. (O-C) residuals of the WZOph $y$-band observations from the theoretical light curve computed for the photometric elements given in Table 17.

Table 19. Adopted photometric elements for WZ Oph. The individual flux and luminosity ratios are based on the mean stellar and orbital parameters.

\begin{tabular}{ll}
\hline \hline$i$ & $89.08 \pm 0.10$ \\
$r_{\mathrm{p}}$ & $0.0952 \pm 0.0008$ \\
$r_{\mathrm{s}}$ & $0.0964 \pm 0.0008$ \\
$r_{\mathrm{p}}+r_{\mathrm{s}}$ & $0.1916 \pm 0.0004$ \\
$k$ & $1.013 \pm 0.015$
\end{tabular}

\begin{tabular}{rrrrr} 
& $y$ & $b$ & $v$ & $u$ \\
$J_{\mathrm{s}} / J_{\mathrm{p}}$ & 0.987 & 0.981 & 0.982 & 0.972 \\
& \pm 15 & \pm 16 & \pm 16 & \pm 17 \\
$L_{\mathrm{S}} / L_{\mathrm{p}}$ & 1.013 & 1.007 & 1.008 & 0.997 \\
& \pm 30 & \pm 27 & \pm 26 & \pm 37 \\
\hline
\end{tabular}

\subsubsection{Abundances for WZOph}

14 FEROS spectra are available of WZ Oph (see Table 1), which allowed us to obtain disentangled spectra of high quality for the abundance studies. Results from the VWA analysis are presented in Table 20. ATLAS9 models were adopted, and the effective temperatures, surface gravities and rotational velocities listed in Table 21 were used, together with microturbulence velocities of $1.7 \mathrm{~km} \mathrm{~s}^{-1}$ for both components, calculated from the calibration by Edvardsson et al. (1993). This choice of 

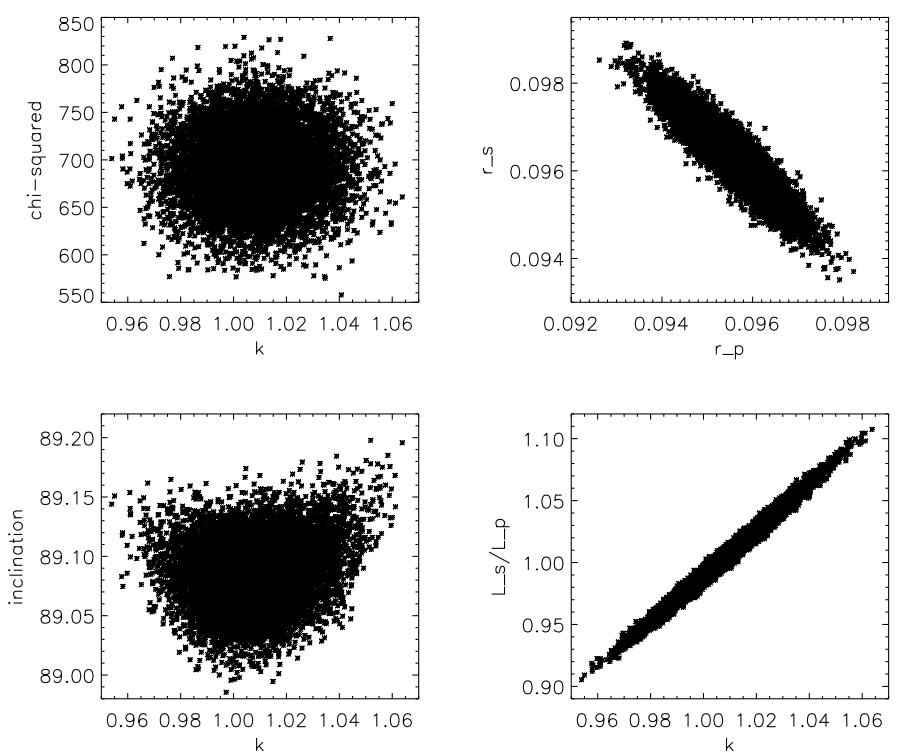

Fig. 16. Best fitting parameter values for the 10000 synthetic WZOph $y$ light curves created for the Monte Carlo analysis.
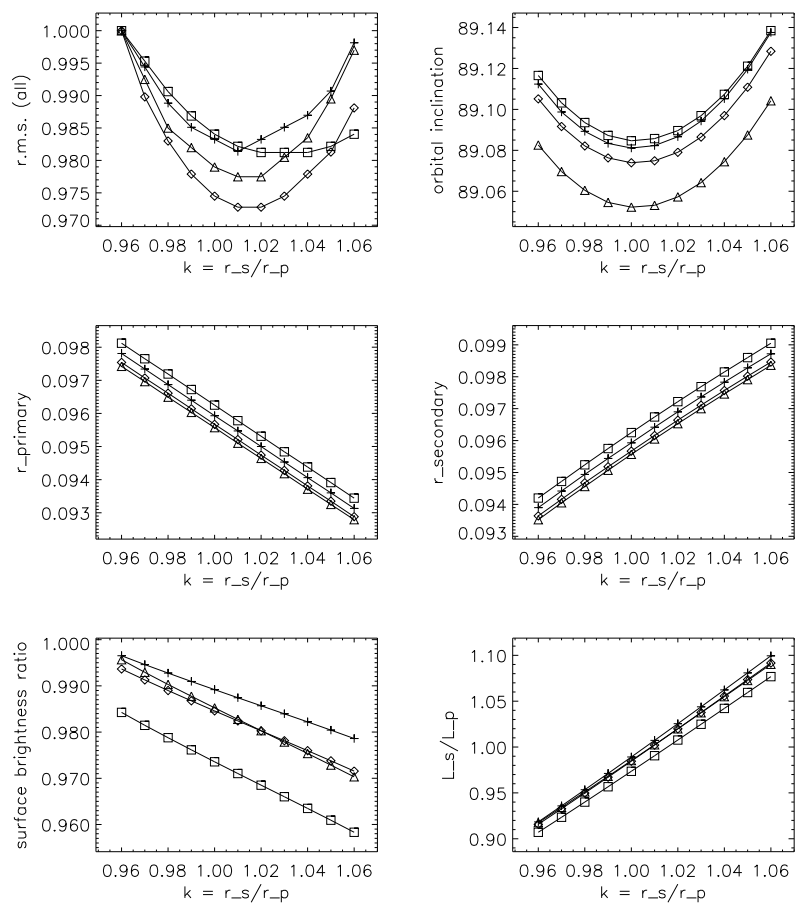

Fig. 17. EBOP solutions for WZOph for a range of fixed $k$ values. Linear limb-darkening coefficients by van Hamme (1993) were adopted. The upper left panel shows normalized rms errors of the fit to the observations. Symbols are: cross $y$; diamond $b$; triangle $v$; square $u$.

parameters is supported by the spectroscopic analyses, since we see no dependence on equivalent width and excitation potential for abundances determined from individual lines. The precision of the temperatures $( \pm 100 \mathrm{~K})$ could not be improved from the abundance analysis.

As seen, a robust $[\mathrm{Fe} / \mathrm{H}]$ is obtained with identical results for $\mathrm{Fe}$ I and Fe II lines from both components, also if MARCS models are used. Changing model temperatures by $\pm 100 \mathrm{~K}$ modifies $[\mathrm{Fe} / \mathrm{H}]$ from the $\mathrm{Fe} I$ lines by $\pm 0.06 \mathrm{dex}$, whereas the Fe II results are nearly unchanged. If $0.5 \mathrm{~km} \mathrm{~s}^{-1}$ higher microturbulence velocities are adopted, $[\mathrm{Fe} / \mathrm{H}]$ decreases by about 0.04 dex for
Table 20. Abundances ([El./H]) for the primary and secondary components of WZOph. $\mathrm{N}$ is the number of lines used per ion.

\begin{tabular}{lccrccr}
\hline \hline & \multicolumn{3}{c}{ Primary } & \multicolumn{3}{c}{ Secondary } \\
Ion & {$[$ El./H] } & rms & $N$ & {$[$ El./H] } & rms & $N$ \\
\hline Na I & -0.32 & 0.05 & 4 & -0.35 & 0.05 & 3 \\
Si I & -0.19 & 0.12 & 20 & -0.18 & 0.09 & 16 \\
Ca I & -0.21 & 0.13 & 11 & -0.36 & 0.18 & 9 \\
Sc II & -0.28 & 0.14 & 5 & -0.30 & 0.07 & 5 \\
Ti I & -0.32 & 0.23 & 3 & -0.26 & 0.20 & 3 \\
Ti II & -0.12 & 0.03 & 4 & -0.17 & 0.10 & 4 \\
V I & +0.03 & 0.25 & 10 & +0.06 & 0.16 & 3 \\
Cr I & -0.36 & 0.25 & 11 & -0.39 & 0.25 & 10 \\
Cr II & -0.17 & 0.17 & 8 & -0.14 & 0.13 & 6 \\
Mn I & -0.54 & 0.27 & 9 & -0.64 & 0.22 & 5 \\
Fe I & -0.27 & 0.12 & 60 & -0.28 & 0.16 & 74 \\
Fe II & -0.28 & 0.04 & 9 & -0.27 & 0.08 & 9 \\
Co I & +0.08 & 0.13 & 4 & -0.18 & 0.31 & 2 \\
Ni I & -0.25 & 0.23 & 13 & -0.25 & 0.16 & 15 \\
\hline
\end{tabular}

both neutral and ionized lines. Taking these contributions into account, we find $[\mathrm{Fe} / \mathrm{H}]=-0.27 \pm 0.07$ for WZ Oph.

Excluding ions with only a few lines measured, we find that within the uncertainties identical abundances are derived for $\mathrm{Cr}$ and $\mathrm{Ni}$, noting that $\mathrm{Cr}$ II deviates, as seen for the Sun (Table 4), although our abundances are relative so that the effect should cancel out. The $\alpha$-elements $\mathrm{Si}$ and $\mathrm{Ca}$ seem to be enhanced by about 0.1 dex, whereas $\mathrm{Na}$ and $\mathrm{Ti}$ (with only few lines measured) appear normal. The Si result may perhaps be questioned, as in the case of Cr II above, since Si I gave a low abundance for the Sun. For Mn, which together with $\mathrm{Al}$ is expected to anticorrelate with $\alpha$-elements, we actually derive a rather low abundance of $-0.54 \pm 0.13$, taking into account temperature and microturbulence uncertainties as above. Unfortunately, problems with the Mn I line were also seen for the Sun. We tend, however, to conclude, that a slight $\alpha$-enhancement of about 0.1 dex may be present. Finally, we derive an abundance for $\mathrm{V}$ close to solar.

As for AD Boo and VZ Hya, metallicities have also been derived from the disentangled CfA spectra. Surface gravities were fixed as above, and synchronous rotation of the components was adopted. The best fits are obtained for $[\mathrm{M} / \mathrm{H}]=-0.14$ and $T_{\text {eff }}=6280 \mathrm{~K}$ (primary), and $[\mathrm{M} / \mathrm{H}]=-0.29$ and $T_{\text {eff }}=6250 \mathrm{~K}$ (secondary), but as in the other two binaries there is a strong correlation between metallicity and temperature, and the determinations are therefore quite uncertain. Forcing the metallicities of the components to be identical, we derive $[\mathrm{M} / \mathrm{H}]=-0.23$ and temperatures of 6230 and $6310 \mathrm{~K}$ for the components, in good agreement with the FEROS abundance result and in reasonable agreement with the photometric temperatures. Estimated uncertainties of metallicities and temperatures from this method are (at least) 0.1 dex and $100 \mathrm{~K}$, respectively.

TODCOR analyses of the observed (composite) CfA spectra for a range of fixed template metallicities yield an estimate of $[\mathrm{M} / \mathrm{H}]=-0.27$, assuming temperatures of 6165 and $6115 \mathrm{~K}$ and constraining the results for the components to be identical.

Finally, the calibration by Holmberg et al. (2007) and the dereddened $u v b y$ indices yield $[\mathrm{Fe} / \mathrm{H}]=-0.17 \pm 0.11$ and $-0.24 \pm$ 0.12 for the primary and secondary components, respectively, also in good agreement with the FEROS result, which we adopt for WZOph. 


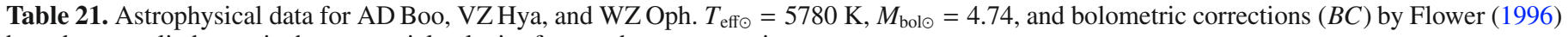
have been applied. $v_{\text {sync }}$ is the equatorial velocity for synchronous rotation.

\begin{tabular}{|c|c|c|c|c|c|c|}
\hline & \multicolumn{2}{|c|}{ AD Boo } & \multicolumn{2}{|c|}{ VZ Hya } & \multicolumn{2}{|c|}{ WZ Oph } \\
\hline & Primary & Secondary & Primary & Secondary & Primary & Secondary \\
\hline \multicolumn{7}{|c|}{ Absolute dimensions: } \\
\hline$M / M_{\odot}$ & $1.414 \pm 0.009$ & $1.209 \pm 0.006$ & $1.271 \pm 0.009$ & $1.146 \pm 0.006$ & $1.227 \pm 0.007$ & $1.220 \pm 0.006$ \\
\hline$R / R_{\odot}$ & $1.612 \pm 0.014$ & $1.216 \pm 0.010$ & $1.314 \pm 0.005$ & $1.112 \pm 0.007$ & $1.401 \pm 0.012$ & $1.419 \pm 0.012$ \\
\hline $\log g(\mathrm{cgs})$ & $4.173 \pm 0.008$ & $4.351 \pm 0.007$ & $4.305 \pm 0.005$ & $4.405 \pm 0.006$ & $4.234 \pm 0.008$ & $4.221 \pm 0.008$ \\
\hline$v \sin i\left(\mathrm{~km} \mathrm{~s}^{-1}\right)$ & $38 \pm 2$ & $37 \pm 5$ & $21 \pm 2$ & $20 \pm 3$ & $18 \pm 1$ & $17 \pm 1$ \\
\hline$v_{\text {sync }}\left(\mathrm{km} \mathrm{s}^{-1}\right)$ & $39.4 \pm 0.4$ & $29.7 \pm 0.2$ & $22.9 \pm 0.1$ & $19.4 \pm 0.1$ & $16.9 \pm 0.1$ & $17.2 \pm 0.1$ \\
\hline \multicolumn{7}{|c|}{ Photometric data: } \\
\hline$V \dagger$ & $9.752 \pm 0.008$ & $10.721 \pm 0.013$ & $9.442 \pm 0.008$ & $10.054 \pm 0.012$ & $9.856 \pm 0.017$ & $9.841 \pm 0.017$ \\
\hline$(b-y) \dagger$ & $0.302 \pm 0.006$ & $0.371 \pm 0.007$ & $0.281 \pm 0.005$ & $0.333 \pm 0.005$ & $0.362 \pm 0.005$ & $0.370 \pm 0.005$ \\
\hline$m_{1} \dagger$ & $0.183 \pm 0.011$ & $0.198 \pm 0.011$ & $0.165 \pm 0.008$ & $0.169 \pm 0.008$ & $0.149 \pm 0.008$ & $0.141 \pm 0.008$ \\
\hline$c_{1} \dagger$ & $0.464 \pm 0.015$ & $0.340 \pm 0.016$ & $0.420 \pm 0.009$ & $0.329 \pm 0.010$ & $0.369 \pm 0.011$ & $0.382 \pm 0.012$ \\
\hline$E(b-y)$ & \multicolumn{2}{|c|}{$0.025 \pm 0.015$} & \multicolumn{2}{|c|}{$0.020 \pm 0.020$} & \multicolumn{2}{|c|}{$0.033 \pm 0.012$} \\
\hline$T_{\text {eff }}$ & $6575 \pm 120$ & $6145 \pm 120$ & $6645 \pm 150$ & $6290 \pm 150$ & $6165 \pm 100$ & $6115 \pm 100$ \\
\hline$M_{\mathrm{bol}}$ & $3.14 \pm 0.08$ & $4.05 \pm 0.09$ & $3.54 \pm 0.10$ & $4.14 \pm 0.10$ & $3.73 \pm 0.07$ & $3.74 \pm 0.07$ \\
\hline $\log L / L_{\odot}$ & $0.64 \pm 0.03$ & $0.28 \pm 0.04$ & $0.48 \pm 0.04$ & $0.24 \pm 0.04$ & $0.41 \pm 0.03$ & $0.40 \pm 0.03$ \\
\hline$B C$ & 0.01 & -0.03 & 0.02 & -0.01 & -0.02 & -0.03 \\
\hline$M_{V}$ & $3.13 \pm 0.08$ & $4.08 \pm 0.09$ & $3.52 \pm 0.10$ & $4.15 \pm 0.10$ & $3.75 \pm 0.07$ & $3.77 \pm 0.07$ \\
\hline$V_{0}-M_{V}$ & $6.51 \pm 0.11$ & $6.54 \pm 0.11$ & $5.83 \pm 0.13$ & $5.82 \pm 0.14$ & $5.96 \pm 0.09$ & $5.94 \pm 0.09$ \\
\hline Distance (pc) & $201 \pm 10$ & $203 \pm 10$ & $147 \pm 9$ & $146 \pm 9$ & $156 \pm 7$ & $154 \pm 7$ \\
\hline \multicolumn{7}{|l|}{ Abundance: } \\
\hline
\end{tabular}

$\dagger$ Not corrected for interstellar absorption/reddening.

\section{Absolute dimensions}

Absolute dimensions for AD Boo, VZ Hya, and WZ Oph are presented in Table 21, as calculated from the new spectroscopic and photometric elements given in Tables 5, 9, 11, 14, 16, and 19.

As seen, masses and radii precise to $0.5-0.7 \%$ and $0.4-0.9 \%$, respectively, have been established for the binary components. For AD Boo, the masses agree well with those determined by Popper (1998a) but are significantly more accurate, whereas those by Lacy (1997a) are about 2\% higher. Both studies report radii in agreement with ours. Popper $(1965,1980)$ reached masses and radii accurate to about $3 \%$ for VZ Hya, and within this uncertainty his results agree with ours. The less massive secondary component of WZ Oph appears slightly larger and more luminous but cooler than the primary. However, within the uncertainties the two components can be considered identical. On average, we obtain masses and radii for WZ Oph which are about $8 \%$ and $5 \%$ larger, respectively, than those given by Popper (1965), and they are far more accurate.

The uvby indices for the components of AD Boo, VZ Hya, and WZ Oph, included in Table 21, were calculated from the the combined indices of the systems at phase 0.25 (CVG08) and the luminosity ratios between their components (Tables 9, 14, 19). The $E(b-y)$ interstellar reddenings, also given in Table 21, were determined from $u v b y \beta$ standard photometry and other sources, as described in more detail in Sect. 4.1.

The adopted effective temperatures listed in Table 21 were calculated from the calibration by Holmberg et al. (2007) assuming the final $[\mathrm{Fe} / \mathrm{H}]$ abundances. The uncertainties $(100-150$ $\mathrm{K})$ include those of the $u v b y$ indices, $E(b-y),[\mathrm{Fe} / \mathrm{H}]$, and the calibration itself. Within errors, temperatures from the $(b-y)$, $c_{1}$ calibration by Alonso et al. (1996) and the $b-y$ calibration by Ramírez \& Meléndez (2005) agree, but for AD Boo we note that both calibrations lead to a temperature difference between the components that is about $120 \mathrm{~K}$ higher. A similar trend was noticed by Holmberg et al. (2007). The empirical flux scale by Popper (1980) and the $y$ flux ratios between the components (Tables 9, 14, 19) yield well established temperature differences of $450 \mathrm{~K}$ (AD Boo), $335 \mathrm{~K}$ (VZ Hya), and $20 \mathrm{~K}$ (WZ Oph), supporting the temperature calibration we have chosen. Details on temperatures derived as part of the TODCOR analyses of the observed CfA spectra (assuming the final metallicities) and the abundance analyses of the disentangled CfA spectra are given in Sect. 3. In general, the results agree well with those included in Table 21, except for WZ Oph where slightly higher temperatures were obtained from the analysis of the disentangled spectra.

The distances to the binaries were calculated from the "classical" relation,

$$
\begin{aligned}
V_{0}-M_{V}= & \left(V-A_{V}\right)-M_{\mathrm{bol} \odot}+5 \log \left(R / R_{\odot}\right) \\
& +10 \log \left(T_{\mathrm{eff}} / T_{\mathrm{eff} \odot}\right)+B C
\end{aligned}
$$

adopting the solar values and bolometric corrections given in Table 21, and $A_{V} / E(b-y)=4.27$. As seen, the distances have been established to 5-6\%, accounting for all error sources and including the use of other $B C$ scales (e.g. Code et al. 1976; Bessell et al. 1998; Girardi et al. 2002). For AD Boo and VZHya, the empirical $K$ surface brightness $-T_{\text {eff }}$ relation by Kervella et al. (2004) leads to nearly identical and perhaps even more precise distances; see Southworth et al. (2005), for details. $K$ photometry outside eclipses is unfortunately not available for WZ Oph. VZ Hya and WZ Oph are included in the Hipparcos catalog (ESA 1997), which gives parallaxes of $5.03 \pm 1.34$ mas and $7.99 \pm 1.37$ mas, respectively, corresponding to distances of $199 \pm 53$ and $125 \pm 21$ pc. Matching the shorter Hipparcos distance for WZ Oph would require lower temperatures, whereas higher interstellar absorption would imply higher temperatures and therefore a nearly unchanged distance. WZ Oph therefore 
does not formally belong to the group of eclipsing binaries within 125 pc discussed by Popper (1998b), which could be of use to improve the radiative flux scale, provided more precise astrometric parallaxes were available.

\subsection{Interstellar reddening and absorption}

The interstellar reddenings of AD Boo, VZHya, and WZOph have been determined as follows:

For AD Boo, we adopt an interstellar reddening of $E(b-y)=$ $0.025 \pm 0.015$, which is derived from the calibration by Olsen (1988) and the standard $u v b y \beta$ indices at phase 0.0, where a significant fraction of the primary component is eclipsed. Ideally, indices at phase 0.5 , where the secondary component is totally eclipsed, should be used, but such $\beta$ observations are unfortunately not available. Combining instead theoretical $\beta$ values (Moon \& Dworetsky 1985) and the individual uvby indices, reddenings of $E(b-y) \approx 0.01 \pm 0.02$ are obtained for both components. Alternatively, $2 \mathrm{MASS} J H K_{\mathrm{s}}$ photometry for stars within $1^{\circ}$ of AD Boo on the sky and closer than 200 pc show a mean absorption of $A_{V}=0.18 \pm 0.04$, corresponding to $E(b-y)=$ $0.04 \pm 0.01$ (Knude, private communication). Finally, the model by Hakkila et al. (1997) yields $A_{V}=0.10$ or $E(b-y)=0.02$, in the direction of and at the distance of AD Boo, and the Schlegel et al. (1998) dust maps give the same amount of total extinction.

For VZ Hya, the calibration by Olsen and the standard $u v b y \beta$ indices at phase 0.5 , where the secondary is nearly totally eclipsed, lead to negligible interstellar reddening, as do the indices at phase 0.0 , where a significant fraction of the primary component is eclipsed. On the other hand, 2MASS $J H K_{\mathrm{s}}$ photometry for stars within $30^{\prime}$ of VZ Hya on the sky and closer than $150 \mathrm{pc}$ show a mean absorption of $A_{V}=0.17 \pm 0.06$, corresponding to $E(b-y)=0.040 \pm 0.014$. Furthermore, the model by Hakkila et al. also yields $A_{V}=0.17$ in the direction of and at the distance of VZHya, whereas the Schlegel et al. dust maps give a total extinction of $A_{V}=0.12$, corresponding to $E(b-y)=0.028$. To encompass the different results we have adopted $E(b-y)=0.02 \pm 0.02$.

For WZ Oph we adopt an interstellar reddening of $E(b-y)=$ $0.033 \pm 0.012$, which is derived from the calibration by Olsen and the standard $u v b y \beta$ indices outside of eclipse, which represent the nearly identical components well. 2MASS $J H K_{\mathrm{s}}$ photometry for stars within $30^{\prime}$ of WZ Oph on the sky and closer than 180 pc show a mean absorption of $A_{V}=0.10 \pm 0.04$, corresponding to $E(b-y)=0.024 \pm 0.009$. In the direction and at the distance of WZ Oph, the model of Hakkila et al. yields a very high absorption of $A_{V}=0.52$, whereas the Schlegel et al. dust maps give of total extinction of $A_{V}=0.28$ or $E(b-y)=0.066$.

\section{Discussion}

In the following, we compare the new dimensions obtained for AD Boo, VZHya, and WZ Oph with properties predicted from some of the latest stellar evolutionary models. Below, we first briefly present the key ingredients of the selected grids, referring to the original papers for full descriptions.

The confrontation between observations and theory for these three systems can be constrained better than in most previous studies, first of all because our $[\mathrm{Fe} / \mathrm{H}]$ determinations and abundance results for other heavy elements are probably among the most detailed done to date for binaries. This means that we can select models not only with respect to mass, but also with respect to $Z$. On the other hand, for a given mass and $Z$, the observable properties of models at a given age such as radius, effective temperature, and luminosity still depend on the adopted input physics, including the treatment of core and envelope convection, diffusion, etc., and on the assumed element mixture and $Y, Z$ relation as well. In order to constrain such free model parameters, accurate results from many binaries are required, supplemented by other results such as those from asteroseismology and cluster analysis. We will focus on the three binaries studied here; a larger sample will be dealt with in forthcoming papers, cf. Fig. 1.

From the binary perspective, masses and radii are the most direct parameters available, free of any scale dependent calibrations. The $M-R$ plane is therefore well suited for isochrone tests, especially when the binary components are significantly different. In addition, the $T_{\text {eff }}-R$ or $T_{\text {eff }}-\log (g)$ planes allow tests of model temperatures, which for a given mass and $Z$ depend, e.g., on the abundance mixture, $Y$, core overshoot treatment, and surface convection efficiency. In our case, the binary temperatures are based on several calibrations and methods, and furthermore the temperature difference between the components is well constrained from the light curves; cf. Sect. 2.5. Adopting the usual assumption of coeval formation from the same raw material, identical ages must be reached in these planes, as well as in the $M-\log (L)$ plane.

\subsection{Summary of models}

As a reference, we have adopted the $Y^{2}$ (Yonsei-Yale) evolutionary tracks and isochrones by Demarque et al. (2004) ${ }^{9}$, which are based on up-to-date input physics and include an improved core overshoot treatment where $\Lambda_{O S}=\lambda_{o v} / H_{\mathrm{p}}$ depends on mass, and which also takes into account the composition dependence of $M_{\text {crit }}^{\text {conv } 10}$ The mixing length parameter in convective envelopes is calibrated using the Sun, and is held fixed at $l / H_{\mathrm{p}}=1.7432$. Helium diffusion has been included. All tracks are started at the pre-main-sequence birth line. Tracks and isochrones are available for a broad range of masses, ages, and heavy element abundances. The enrichment law $Y=0.23+2 Z$ is adopted, together with the solar mixture by Grevesse et al. (1996), leading to ( $X$, $Y, Z)_{\odot}=(0.71564,0.26624,0.01812)$. Models with enhanced $\alpha$-element abundances are also included; see Kim et al. (2002) for further details. We have used the abundance, mass, and age interpolation routines provided by the $Y^{2}$ group.

Another recent example of stellar evolution calculations is given by the extensive Victoria-Regina grids (VandenBerg et al. 2006) ${ }^{11}$, which adopt a somewhat different physically based description of the core overshoot with $F_{\text {over }}$ depending on mass and metallicity and calibrated observationally via cluster CMD's. Diffusive processes are not treated. All tracks have been computed from the pre-main-sequence stage. The VRSS grids (scaled-solar) used here adopt $Y=0.23544+2.2 Z$ and the solar mixture by Grevesse \& Noels (1993), leading to $(X, Y, Z)_{\odot}=$ $(0.7044,0.2768,0.0188)$. The mixing length parameter in convective envelopes is calibrated using the Sun and held at $\alpha_{M L T}=$ 1.90. For further details on the chemical abundances adopted for the VR0A, VR2A, and VR4A grids (different $[\alpha / \mathrm{Fe}]$ ) we refer the reader to VandenBerg et al. (2000). We have used the

\footnotetext{
9 http://www . astro.yale.edu/demarque/yystar.html

10 Defined as "the mass above which stars continue to have a substantial convective core even after the end of the pre-MS phase".

11 http://www1. cadc-ccda.hia-iha.nrc-cnrc.gc.ca/cvo/ community/VictoriaReginaModels/
} 


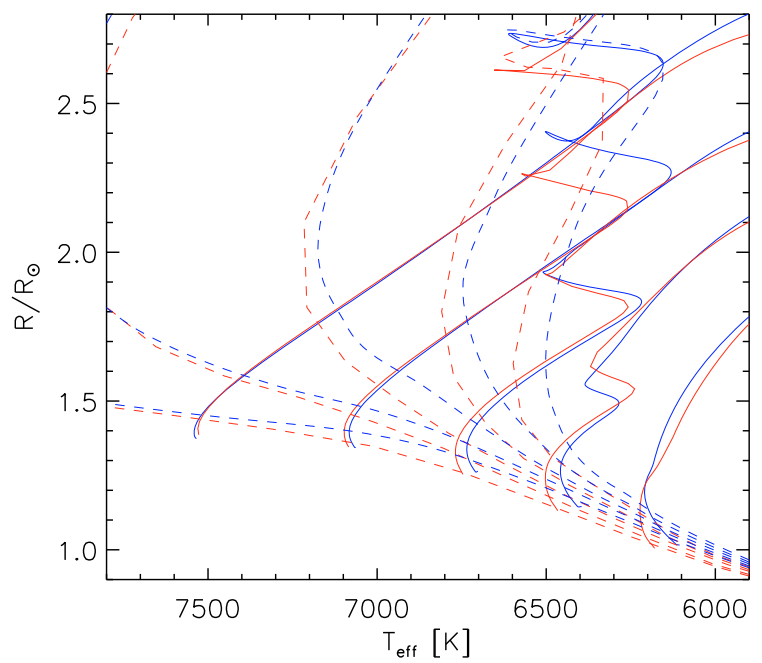

Fig. 18. Comparison between $Y^{2}$ (red) and Victoria-Regina VRSS (blue) models for $[\mathrm{Fe} / \mathrm{H}]=-0.190$ and $[\alpha / \mathrm{Fe}]=0.0 . Y^{2}:(X, Y, Z)$ $=(0.73400,0.25400,0.01200)$. VRSS: $(X, Y, Z)=(0.72456,0.26294$, 0.01250 ). Tracks (solid lines) for $1.1,1.2,1,3.1 .4$, and $1.5 M_{\odot}$ and isochrones (dashed) between 0.5 and $2.5 \mathrm{Gyr}$ (step $0.5 \mathrm{Gyr}$ ) are shown.

isochrone interpolation routines provided by the Victoria-Regina group; track interpolations have not been done.

A third source of modern evolutionary tracks and isochrones for specific masses and ages is the BaSTI database (Pietrinferni et al. 2004) $)^{12}$, available for several compositions. It is based on a recent version of the FRANEC evolutionary code (Degl'Innocenti et al. 2007). Models without and with core overshoot are available; in the latter case the choice of $\lambda_{\text {ov }}$ depends on mass but not on metallicity. $Y=0.245+1.4 Z$ and the solar mixture by Grevesse \& Noels (1993) are used, and the mixing length parameter in convective envelopes is calibrated using the Sun and held fixed at $l / H_{\mathrm{p}}=1.913$. Mass loss is included, but atomic diffusion is taken into account only in the standard solar model. All tracks have been computed from the pre-mainsequence phase.

A comparison of the main sequence models between 1.1 and $1.5 M_{\odot}$ for identical $[\mathrm{Fe} / \mathrm{H}]$ values close to those of our stars reveals some differences between the three grids described above. This is exemplified in Figs. 18 and 19. In the lower main-sequence band, the $Y^{2}$ and the Victoria-Regina tracks agree quite well. The isochrones are slightly shifted, and for part of the mass interval the effect increases in the upper part of the main-sequence band due to differences in the treatment of core overshoot and the ramping procedure adopted. The BaSTI tracks differ somewhat in shape and location, and consequently significant differences are seen in the isochrones as well.

Before focusing on the confrontation between binary observations and models, we point out that we have not considered here models based on scaling of the most recent solar mixture (Grevesse et al. 2007), which implies a significantly lower metallicity of $Z_{\odot} \approx 0.013$ and a different conversion between $[\mathrm{Fe} / \mathrm{H}]$ and $Z$. At present, solar models computed with these abundances disagree with precise results inferred from helioseismology, and the cause(s) have yet to be identified (e.g. Basu \& Antia 2007).

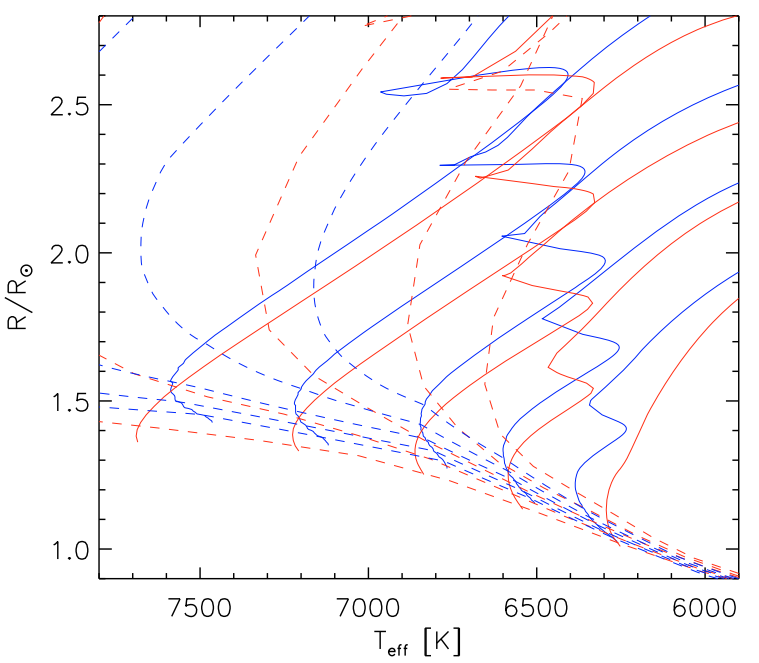

Fig. 19. Comparison between $Y^{2}$ (red) and BaSTI core overshoot (blue) models for $[\mathrm{Fe} / \mathrm{H}]=-0.253$ and $[\alpha / \mathrm{Fe}]=0.0 . Y^{2}:(X, Y, Z)=(0.73865$, $0.25090,0.01045)$. BaSTI: $(X, Y, Z)=(0.7310,0.2590,0.0100)$. Tracks (solid lines) for 1.1, 1.2, 1.3, 1.4, and $1.5 M_{\odot}$ and isochrones (dashed) between 0.5 and $2.5 \mathrm{Gyr}$ (step $0.5 \mathrm{Gyr}$ ) are shown.

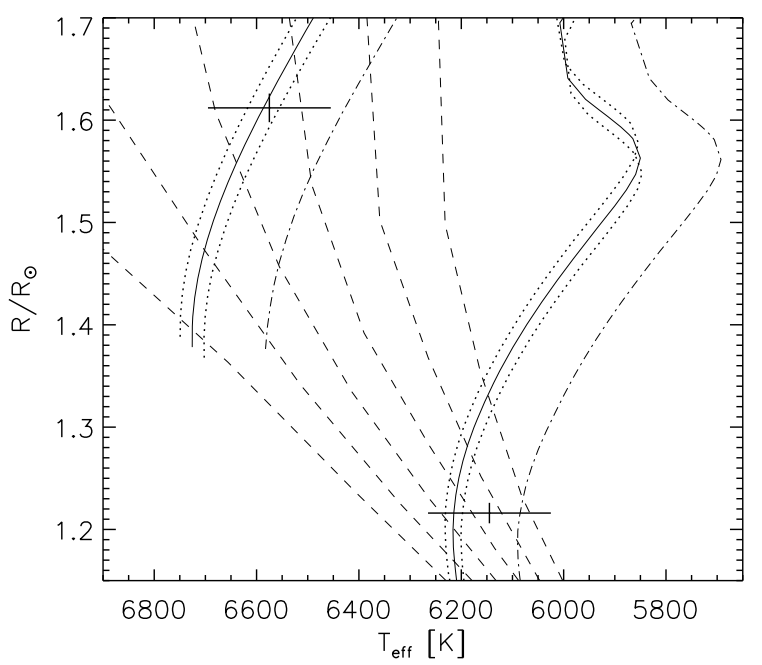

Fig. 20. AD Boo compared with $Y^{2}$ models for $(X, Y, Z)=$ $(0.7028,0.2748,0.0224)$, equivalent to the measured abundance $[\mathrm{Fe} / \mathrm{H}]=+0.10 \pm 0.15$ for $[\alpha / \mathrm{Fe}]=0.0$. Tracks for the component masses (solid lines) and isochrones from 0.5 to $3.0 \mathrm{Gyr}$ (dashed; step $0.5 \mathrm{Gyr}$ ) are shown. The uncertainty in the location of the tracks coming from the mass errors are indicated (dotted lines). To illustrate the effect of the abundance uncertainty, tracks for $[\mathrm{Fe} / \mathrm{H}]=+0.25$ and $[\alpha / \mathrm{Fe}]=0.0$ (dash-dot), corresponding to $(X, Y, Z)=(0.6785,0.2910$, $0.0305)$, are included.

\subsection{Comparison with the binary results}

For AD Boo, comparisons between the absolute dimensions and $Y^{2}$ models calculated for the measured $[\mathrm{Fe} / \mathrm{H}]$ are shown in Figs. 20-22. As seen in Fig. 21, an isochrone corresponding to $\sim 1.75$ Gyr would fit both components very well, and for the primary component this age is also deduced from Figs. 20 and 22. The abundance uncertainty of \pm 0.15 dex translates to an age uncertainty of about $\pm 0.15 \mathrm{Gyr}$, comparable to that from mass and radius uncertainties. The model tracks fit the components well but predict a $\sim 100 \mathrm{~K}$ lower temperature difference between the components than established from both photometry and the

12 http://www.te.astro.it/BASTI/index.php 


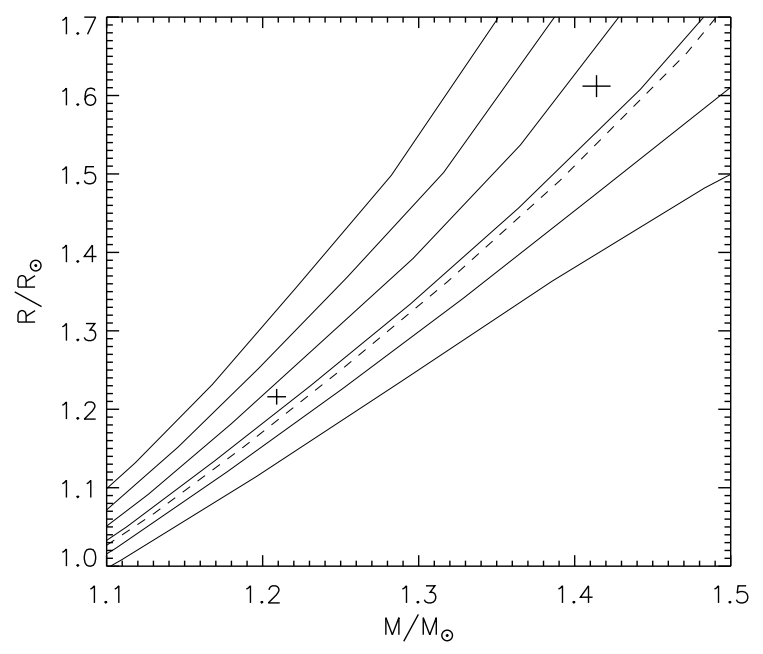

Fig. 21. AD Boo compared with $Y^{2}$ models for $(X, Y, Z)=$ $(0.7028,0.2748,0.0224)$, equivalent to $[\mathrm{Fe} / \mathrm{H}]=+0.10$ for $[\alpha / \mathrm{Fe}]=0.0$. Isochrones from 0.5 to $3.0 \mathrm{Gyr}$ (step $0.5 \mathrm{Gyr}$ ) are shown. The $1.5 \mathrm{Gyr}$ isochrone for $[\mathrm{Fe} / \mathrm{H}]=+0.25$ and $[\alpha / \mathrm{Fe}]=0.0$ (dashed), corresponding to $(X, Y, Z)=(0.6785,0.2910,0.0305)$, is included for comparison.

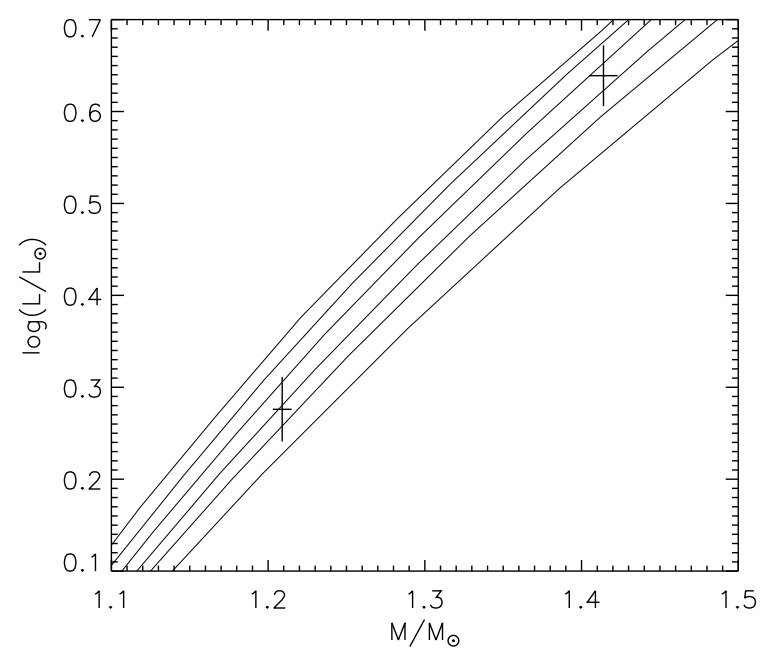

Fig. 22. AD Boo compared with $Y^{2}$ models for $(X, Y, Z)=(0.7028$, $0.2748,0.0224)$, equivalent to $[\mathrm{Fe} / \mathrm{H}]=+0.10$ for $[\alpha / \mathrm{Fe}]=0.0$. Isochrones from 0.5 to $3.0 \mathrm{Gyr}$ (step $0.5 \mathrm{Gyr}$ ) are shown.

empirical flux scale; see Sect. 4. Consequently, the predicted model luminosity of the secondary is also marginally higher than observed (Fig. 22). If real, the slight discrepancy may be related to the activity of the secondary component. The same picture is seen for Victoria-Regina models, which give a slightly lower age of $\sim 1.5$ Gyr. On the other hand, the BaSTI models fit AD Boo less well. For the observed masses and radii, they predict quite different ages of about 2.25 and 1.75 Gyr for the primary and secondary, respectively. This is probably related to the different shape of the BaSTI tracks (Fig. 19) in the lower main-sequence region.

Figures 23-25 compare the absolute dimensions for VZ Hya with the $Y^{2}$ models calculated for the measured [Fe/H]. As seen in Fig. 24, an isochrone for an age of 1.25 Gyr fits both components within errors, although a slightly steeper isochrone slope would formally improve the fit still more. The abundance uncertainty of \pm 0.12 dex corresponds to about \pm 0.25 Gyr in age, similar to the contribution from mass and radius uncertainties. The model tracks are slightly hotter than observed, and consequently

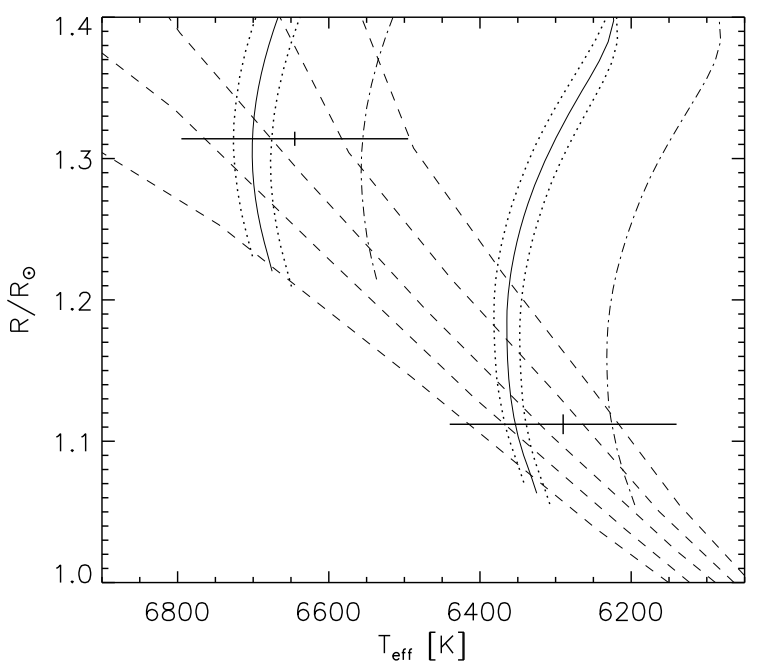

Fig. 23. VZ Hya compared with $Y^{2}$ models for $(X, Y, Z)=(0.73478$, $0.25348,0.01174)$ equivalent to the measured abundance $[\mathrm{Fe} / \mathrm{H}]=$ $-0.20 \pm 0.12$ for $[\alpha / \mathrm{Fe}]=0.0$. Tracks for the component masses (solid lines) and isochrones from 0.5 to $2.5 \mathrm{Gyr}$ (dashed; step $0.5 \mathrm{Gyr}$ ) are shown. The uncertainty in the location of the tracks coming from the mass errors are indicated (dotted lines). To illustrate the effect of the abundance uncertainty, tracks for $[\mathrm{Fe} / \mathrm{H}]=-0.08$ and $[\alpha / \mathrm{Fe}]=0.0$ (dash-dot), corresponding to $(X, Y, Z)=(0.72425,0.26050,0.01525)$, are included.

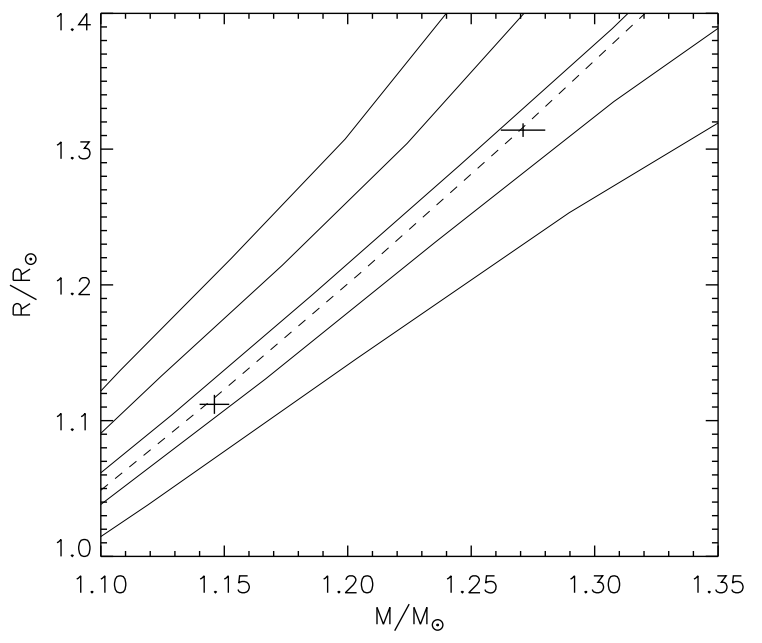

Fig. 24. VZ Hya compared with $Y^{2}$ models for $(X, Y, Z)=$ $(0.73478,0.25348,0.01174)$ equivalent to $[\mathrm{Fe} / \mathrm{H}]=-0.20$. Isochrones from 0.5 to $2.5 \mathrm{Gyr}$ (step $0.5 \mathrm{Gyr}$ ) are shown. The $1.5 \mathrm{Gyr}$ isochrone for $[\mathrm{Fe} / \mathrm{H}]=-0.08$ and $[\alpha / \mathrm{Fe}]=0.0$ (dashed), corresponding to $(X, Y, Z)$ $=(0.72425,0.26050,0.01525)$, is included for comparison.

the ages derived from Figs. 23 and 25 become too high and low, respectively. Both tracks would fit well for a slightly higher $[\mathrm{Fe} / \mathrm{H}]$ of -0.15 , safely within the uncertainty of the measured value. Adopting $[\mathrm{Fe} / \mathrm{H}]=-0.15$, identical ages of $1.25 \mathrm{Gyr}$ are obtained from all three diagrams. Victoria-Regina tracks for the observed $[\mathrm{Fe} / \mathrm{H}]$ fit the components well, being slightly cooler than the $Y^{2}$ tracks. For the observed masses and radii, ages become lower, about 1.0 and 0.7 Gyr for primary and secondary, respectively; they agree within errors. The BaSTI models yield about 1.25 and $0.75 \mathrm{Gyr}$, and in this case the difference is significant.

Whereas the $Y^{2}$ models in general match the observed properties of AD Boo and VZHya well, the situation for WZ Oph is completely different, as seen from Fig. 26. Models for the 


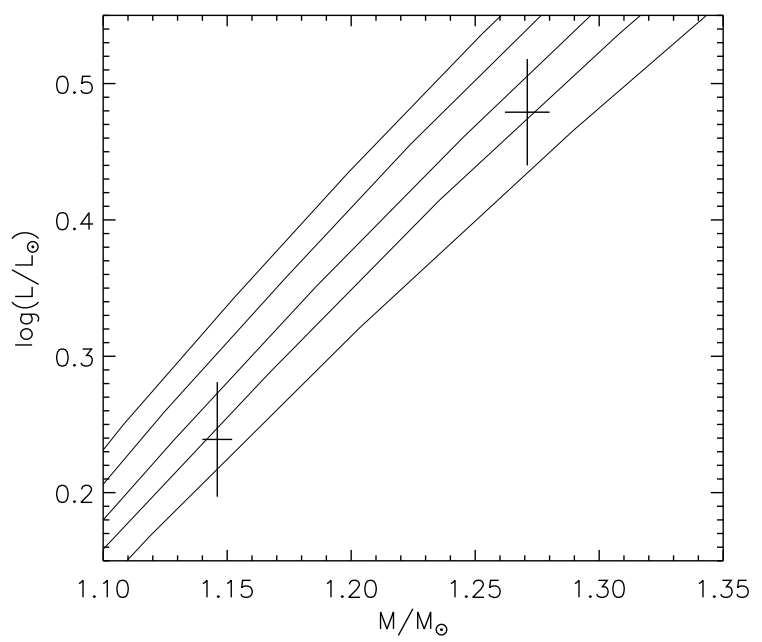

Fig. 25. VZ Hya compared with $Y^{2}$ models for $(X, Y, Z)=(0.73478$, $0.25348,0.01174)$ equivalent to $[\mathrm{Fe} / \mathrm{H}]=-0.20$. Isochrones from 0.5 to $2.5 \mathrm{Gyr}$ (step $0.5 \mathrm{Gyr}$ ) are shown.

measured $[\mathrm{Fe} / \mathrm{H}]$ are much too hot, both for $[\alpha / \mathrm{Fe}]=0.0$ and +0.1 (cf. Sect. 3.3.3), and the same difference is seen for Victoria-Regina and BaSTI models. In order to reproduce WZ Oph for the observed $[\mathrm{Fe} / \mathrm{H}]$, an unrealistically high $[\alpha / \mathrm{Fe}]$ of about +0.5 would be required. Alternatively, the models would need to have a significantly lower helium content and/or an envelope mixing length parameter well below $l / H_{\mathrm{p}}=1.7432$. The latter can not a priori be ruled out, since the photometry and spectroscopy indicate the presence of some degree of surface activity on both components of WZ Oph; see Sect. 3.3.2. It would therefore be of great interest to have available models for the observed $[\mathrm{Fe} / \mathrm{H}]$ and a range of $l / H_{\mathrm{p}}$ values. Such models have been published by Baraffe et al. (1998) ${ }^{13}$ but only for solar metallicity. They suggest that $l / H_{\mathrm{p}} \sim 1$ or even lower would be needed, although models computed specifically for this case would be required in order to evaluate the combined effects on the stellar radii and temperatures in detail. The possible connection between activity and convection, as indicated from studies of solar-type binaries, has been discussed by Clausen et al. (1999), Torres et al. (2006), and others.

As stated in Sect. 4.1, we have adopted an interstellar reddening of $E(b-y)=0.033 \pm 0.012$ for WZ Oph, equivalent to $A_{V}=0.14 \pm 0.05$ for a standard extinction law. There were, however, some indications of higher absorption from other sources. If we therefore somewhat arbitrarily double it and assume $A_{V}=0.28$ and $E(b-y)=0.066$, the effective temperatures derived from the uvby indices increase by about $250 \mathrm{~K}$, i.e. to about $6400 \mathrm{~K}$. This has an effect on the abundances. From the Fe I lines we would derive $[\mathrm{Fe} / \mathrm{H}] \sim-0.13$ for both components, whereas the results from the Fe II lines would be practically unchanged at -0.27 . The photometric determinations from the calibration by Holmberg et al. (2007) would increase by about 0.12 dex for both components. The discrepancy between neutral and ionized $\mathrm{Fe}$ lines argues against the higher temperatures, but assuming them, together with the higher abundance from the Fe I lines, does on the other hand remove the model discrepancies, as seen in Fig. 27. The age of WZ Oph then becomes about 3 Gyr.

An independent check would be to derive intrinsic colours and/or effective temperatures from line-depth ratios measured in the disentangled FEROS spectra, see e.g. Gray (1994), but WZ Oph is unfortunately slightly too hot for the calibrations

13 ftp.ens-lyon.fr

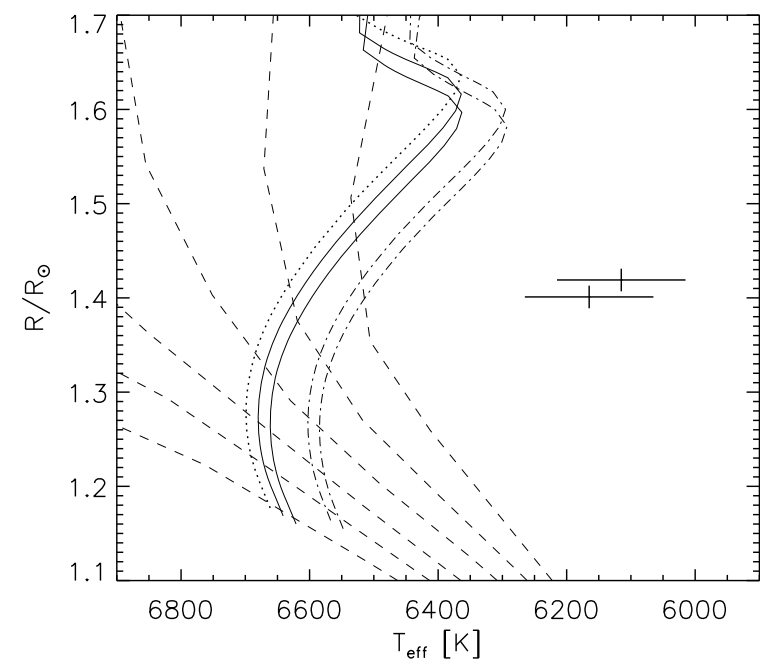

Fig. 26. WZ Oph compared with $Y^{2}$ models for $(X, Y, Z)=$ $(0.73985,0.25010,0.01005)$, equivalent to the measured abundance $[\mathrm{Fe} / \mathrm{H}]-0.27 \pm 0.07$ for $[\alpha / \mathrm{Fe}]=0.0$. Tracks for the component masses (solid lines) and isochrones from 0.5 to $3.0 \mathrm{Gyr}$ (dashed; step $0.5 \mathrm{Gyr}$ ) are shown. The uncertainty in the location of the primary track coming from the mass error is indicated (dotted line). For comparison, tracks for $(X, Y, Z)=(0.7349,0.2534,0.0117)$ and $[\alpha / \mathrm{Fe}]=+0.1$, also equivalent to $[\mathrm{Fe} / \mathrm{H}]=-0.27$, are included (dash-dot).

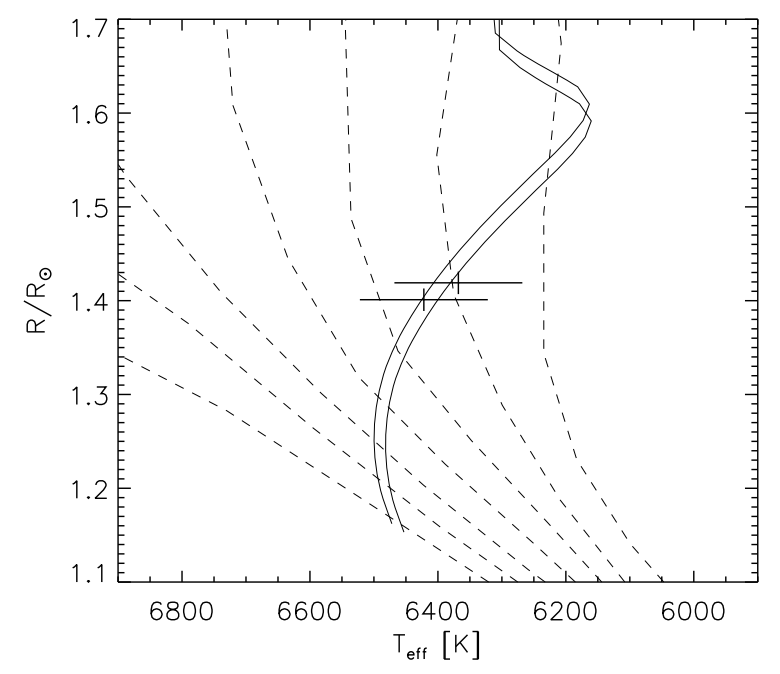

Fig. 27. Assuming $E(b-y)=0.066$ : WZ Oph compared with $Y^{2}$ models for $(X, Y, Z)=(0.72899,0.25734,0.013670)$, equivalent to $[\mathrm{Fe} / \mathrm{H}]=$ -0.13 for $[\alpha / \mathrm{Fe}]=0.0$. Tracks for the component masses (full drawn) and isochrones from 0.5 to $3.5 \mathrm{Gyr}$ (dashed; step $0.5 \mathrm{Gyr}$ ) are shown. See text for details.

available. Adopting a subset of those by Kovtyukh et al. (2003), valid to 6100 or $6150 \mathrm{~K}$, we derive mean effective temperatures close to $6200 \mathrm{~K}$ for both components. The individual results from the 9 (primary) and 16 (secondary) measured line-depth ratios scatter by $100-200 \mathrm{~K}$, and since we are at the edge of the calibrations, the method does not at the moment provide new firm information.

Although we cannot pinpoint the reason for the discrepancy between the observations and the models for WZ Oph, we suspect they are connected to composition (He) or convection anomalies in the stars and/or the determination of interstellar reddening, rather than to physical or numerical problems with the model calculations. This is supported by the general good agreement seen for AD Boo and especially VZ Hya, but detailed 
studies of additional systems similar to these are needed for full clarification. Work on several new F-G type binaries is in progress.

\section{Summary and conclusions}

We have presented precise absolute dimensions and abundances for the three F-type double-lined eclipsing binaries AD Boo, VZHya, and WZ Oph. The results, which are based on stateof-the-art analyses of new photometric and spectroscopic observations, were used to test the recent $Y^{2}$, Victoria-Regina, and BaSTI stellar evolutionary models.

Masses and radii precise to $0.5-0.7 \%$ and $0.4-0.9 \%$ have been established for the binary components. This level can only be reached if excellent observations are available and are analysed carefully using adequate tools. Special care was taken to identify and avoid possible systematic errors. Several methods were applied in order to determine reliable and accurate $(100-150 \mathrm{~K})$ effective temperatures and interstellar extinctions, still a challenging task whose importance can not be overestimated.

System $[\mathrm{Fe} / \mathrm{H}]$ abundances were based on numerous lines and were derived relative to the Sun. We have reached precisions between 0.07 and 0.15 dex. Even having many Fe I and Fe II lines of different strength and excitation potential available, the strong correlation between abundance and effective temperature is difficult to break. $T_{\text {eff }}$ and microturbulence uncertainties contribute significantly to the final abundance precision. Abundances for other heavy elements, based on fewer lines, were also derived; in one case (WZ Oph) indications of a slight $\alpha$-element overabundance is seen. Comparing the results for the three binaries clearly demonstrates that analyses of disentangled spectra are far superior to those of composite spectra, essentially because many more lines become available and blending becomes less of an issue.

AD Boo has components with the greatest difference in mass and radius among well-studied F-type binaries $\left(1.41 M_{\odot}\right.$, $\left.1.61 R_{\odot} ; 1.21 M_{\odot}, 1.22 R_{\odot}\right)$ and an abundance of $[\mathrm{Fe} / \mathrm{H}]=$ +0.10 . Slight surface activity is present on the secondary component. The system is fitted well by $Y^{2}$ and Victoria-Regina evolutionary models for identical component ages (as determined from masses and radii) of 1.75 and $1.5 \mathrm{Gyr}$, respectively. The fact that the secondary appears marginally cooler than the corresponding evolutionary track may be related to mild surface activity. BaSTI models predict different ages of 2.25 and $1.75 \mathrm{Gyr}$ for the components, and therefore do not fit AD Boo as well.

VZHya has somewhat different components $\left(1.27 M_{\odot}\right.$, $\left.1.31 R_{\odot} ; 1.14 M_{\odot}, 1.11 R_{\odot}\right)$ and an abundance of $[\mathrm{Fe} / \mathrm{H}]=$ -0.20 . No signs of surface activity are seen. $Y^{2}$ and VictoriaRegina evolutionary tracks fit VZ Hya well. Nearly identical component ages (as determined from masses and radii) of $1.25 \mathrm{Gyr}$ are obtained from the $Y^{2}$ isochrones, whereas the Victoria-Regina calculations give 1.0 and 0.7 Gyr for the primary and secondary. An even larger difference, 1.25 versus $0.75 \mathrm{Gyr}$, is found when using the BaSTI models.

WZOph consists of two nearly identical components $\left(1.22 M_{\odot}, 1.41 R_{\odot}\right)$. The $[\mathrm{Fe} / \mathrm{H}]$ abundance is -0.27 , and as mentioned above a slight $\alpha$-element overabundance is possible. Photometry and spectroscopy suggest low-level surface activity on both components. Contrary to the case for AD Boo and VZ Hya, neither of the three model grids are able to fit the observations for WZ Oph; models are significantly hotter than observed. We tentatively conclude that this is caused either by
WZ Oph anomalies such as a low He content or decreased envelope convection, or by underestimated interstellar reddening. Further observations will be required to resolve the issue.

We believe our study is among the most detailed carried out to date for main-sequence eclipsing binaries, and that it sets new standards for critical tests of stellar evolutionary models based on accurate binary data. With only three systems available, it is premature to draw firm conclusions and suggest specific model shortcomings and/or improvements. We find, however, better agreement with the $Y^{2}$ and Victoria-Regina models than with the BaSTI models. In contradiction to this, Tomasella et al. (2007) recently found that dedicated BaSTI models, including core overshoot and both helium and heavy element diffusion, represent the components of the young $\sim 1.25 M_{\odot}$ system V505 Per well. Their study is based on new absolute dimensions of extraordinary high formal precision.

As shown in Fig. 1, several additional F-type binaries are available, and we are presently working on re-analyses for some of them, including abundance determinations. We are also conducting a larger program on new F-G type systems exhibiting various levels of surface activity (Clausen et al. 2001), and we expect some very useful insights to come from these studies, and from a parallel program on eclipsing binaries in clusters spanning a wide range in age and metallicity (e.g. Grundahl et al. 2006).

Acknowledgements. The spectroscopic observations at the CfA facilities were obtained with the able help of J. R. Caruso, R. J. Davis, E. Horine, R. D. Mathieu, A. A. E. Milone, J. Peters, and J. M. Zajac. We are grateful to R. J. Davis for maintaining the echelle database at CfA. We thank U. G. Jørgensen for many inspiring discussions and for allowing us to use his MARCS code. A. Kaufer, O. Stahl, S. Tubbesing, and B. Wolf kindly obtained a FEROS spectrum of WZ Oph spectra during Heidelberg/Copenhagen guaranteed time in 1999. We thank H. Hensberge for making his improved version of the MIDAS FEROS package available, and both him and L. Freyhammer for valuable advise and help during the reduction of the spectra. We are grateful to E. Sturm for providing his original disentangling code, and to him and J. D. Pritchard for modifying it for use at Linux/Unix computer systems. J. Knude kindly provided interstellar absorption results from his $J H K_{\mathrm{s}}$ calibration. The projects "Stellar structure and evolution - new challenges from ground and space observations" and "Stars: Central engines of the evolution of the Universe", carried out at Copenhagen University and Aarhus University, are supported by the Danish National Science Research Council. G. T. acknowledges partial support from NSF grants AST-0406183 and AST-0708229, and JA and BN from the Carlsberg Foundation. The following internet-based resources were used in research for this paper: the NASA Astrophysics Data System; the SIMBAD database and the VizieR service operated by CDS, Strasbourg, France; the ar $\chi$ iv scientific paper preprint service operated by Cornell University. This publication makes use of data products from the Two Micron All Sky Survey, which is a joint project of the University of Massachusetts and the Infrared Processing and Analysis Center/ California Institute of Technology, funded by the National Aeronautics and Space Administration and the National Science Foundation.

\section{References}

Alonso, A., Arribas, S., \& Martínez-Roger, C. 1996, A\&A, 313, 873 Andersen, J. 1991, A\&AR, 3, 91

Baraffe, I., Chabrier, G., Allard, F., \& Hauschildt, P. 1998, A\&A, 337, 403

Basu, S., \& Antia, H. M. 2007, [arXiv: astro-ph/07114590]

Bessell, M. S., Castelli, F., \& Plez, B. 1998, A\&A, 333, 231

Bruntt, H., Catala, C., Garrido, R., et al. 2002, A\&A, 389, 345

Bruntt, H., Bikmaev, I. F., Catala, C., et al. 2004, A\&A, 425, 683

Bruntt, H., De Cat, P., \& Aerts, C. 2008, A\&A, 478, 487

Casey, B. W., Mathieu, R. D., Vaz, L. P. R., Andersen, J., \& Suntzeff, N. B. 1998, AJ, 115, 1617

Cester, B., Fedel, B., Giuricin, G., et al. 1978, A\&AS, 32, 351

Claret, A., 2000, A\&A, 363, 1081

Clausen, J. V. 2004, New A Rev., 48, 679

Clausen, J. V., Baraffe, I., Claret, A., \& VandenBerg, D. B. 1999, in Theory and Tests of Convection in Stellar Structure, ed. A. Giménez, E. F. Guinan, \& B. Montesinos, ASP Conf. Ser., 173, 265

Clausen, J. V., Helt, B. E., \& Olsen, E. H. 2001, A\&A, 374, 980 
Clausen, J. V., Vaz, L. P. R., García, J. M., et al. 2008, A\&A, 487, 1081 (CVG08) Code, A. D., Bless, R. C., Davis, J., \& Brown, R. H. 1976, ApJ, 203, 417 Degl'Innocenti, S., Prada Moroni, P. G., Marconi, M., \& Ruoppo, A. 2007, Ap\&SS.tmp..291D (online)

Demarque, P., Woo, J.-H., Kim, Y.-C., \& Yi, S. K. 2004, ApJS, 155, 667

Díaz-Cordovés, J., Claret, A., \& Giménez, A. 1995, A\&AS, 110, 329

ESA 1997, The Hipparcos and Tycho Catalogues, ESA SP-1200

Edvardsson, B., Andersen, J., Gustafsson, B., et al. 1993, A\&A, 275, 101

Etzel, P. B. 1981, in Photometric and Spectroscopic Binary Systems, ed. E. B. Carling, \& Z. Kopal, 111

Flower, P. J. 1996, ApJ, 469, 355

Gray, D. F. 2004, PASP, 106, 1248

Girardi, L., Bertelli, G., Bressan, A., et al. 2002, A\&A, 391, 195

Grevesse, N., \& Noels, A. 1993, Phys. Scr. T47, 133

Grevesse, N., \& Sauval, A. J. 1998, Space Sci. Rev., 85, 161

Grevesse, N., Noels, A., \& Sauval, A. J. 1996, in Cosmic Abundances, ed. S. S. Holt, \& G. Sonneborn (San Francisco: ASP), 117

Grevesse, N., Asplund, M., \& Sauval, A. J. 2007, Space Sci. Rev., 130, 105 Grundahl, F., Arentoft, T., Bruntt, H., et al. 2006, Mem. Soc. Astron. It., 77, 433 Gustafsson, B., Bell, R. A., Eriksson, K., \& Nordlund, Å. 1975 A\&A, 42, 407

Hakkila, J., Myers, J. M., Stidham, B. J., \& Hartmann, D. H. 1997, AJ, 114, 2043 Hall, D. S. 1994, Mem. Soc. Astron. It., 65, 73

Heiter, U., Kupka, F., van’t Menneret, C., et al. 2002, A\&A, 392, 619

Holmberg, J., Nordström, B., \& Andersen, J. 2007, A\&A, 475, 519

Jørgensen, U. G., Johnson, H. R., \& Nordlund, ^̊ 1992, A\&A, 261, 263

Kaufer, A., Stahl, O., Tubbesing, S., et al. 1999, The ESO Messenger, 95, 8

Kaufer, A., Stahl, O., Tubbesing, S., et al. 2000, in Optical and IR Telescope Instrumentation and Detectors, ed. M. Iye, \& A. F. Morwood, Proc. SPIE 4008, 459

Kervella, P., Thévenin, F., Di Folco, E., \& Ségransan, D. 2004, A\&A, 426, 297

Kim, Y.-C., Demarque, P., Yi, S. K., \& Alexander, D. R. 2002, ApJS, 143, 499

Klinglesmith, D. A., \& Sobieski, S. 1970, AJ, 75, 175

Kovtyukh, V. V., Soubiran, C., Belik, S. I., \& Gorlova, N. I. 2003, A\&A, 411, 559

Kupka, F., Piskunov, N., Ryabchikova, T. A., Stempels, H. C., \& Weiss, W. 1999, A\&AS, 138, 119

Kurucz, R. L., 1993, in Light Curve Modelling of Eclipsing Binary Stars, ed. E. F. Milone (Springer Verlag), 93

Lacy, C. H. 1977, ApJS, 34, 479

Lacy, C. H. 1985, Inf. Bull. Var. Stars, 2685

Lacy, C. H. S. 1997, AJ, 113, 1406

Latham, D. W. 1992, in Complementary Approaches to Double and Multiple Star Research, ed. H. A. McAlister, \& W. I. Hartkopf (San Francisco: ASP), IAU Coll. 135, ASP Conf. Ser. 32, 110

Latham, D. W., Nordström, B., Andersen, J., et al. 1996, A\&A, 314, 864

Latham, D. W., Stefanik, R. P., Torres, G., et al. 2002, AJ, 124, 1144

Martynov D. Ya. 1973, in Eclipsing Variable Stars, ed. V. P. Tsesevich, Israel Program for Scientific Translation, Jerusalem

Masana, E., Jordi, C., \& Ribas, I. 2006, A\&A, 450, 735

Moon, T. T., \& Dworetsky, M. M. 1985, MNRAS, 217, 305

Nelson, B., \& Davis, W. 1972, ApJ, 174, 617

Nordlund, A., \& Vaz, L. P. R. 1990, A\&A, 228, 231

Nordström, B., Latham, D. W., Morse, J. A., et al. 1994, A\&A, 287, 338

O'Connell, J. K. 1932, Harvard Bull, No. 889, 7

Olsen, E. H. 1988, A\&A, 189, 173
Padalia, T. D. 1986, Ap\&SS, 119, 395

Padalia, T. D., \& Srivastava, R. K. 1975, Ap\&SS, 35, 349

Pickering, E. C. 1917, Harvard Circ. No. 201

Pietrinferni, A., Cassisi, S., \& Salaris, M. 2004, Mem. Soc. Astron. Italiana, 75, 170

Popper, D. M. 1965, ApJ, 141, 126

Popper, D. M. 1976, Ap\&SS, 45, 391

Popper, D. M. 1980, ARA\&A, 18, 115

Popper, D. M. 1998a, AJ, 115, 338

Popper, D. M. 1998b, PASP, 110, 919

Popper, D. M., \& Etzel, P. B. 1981, AJ, 86, 102

Press, W. H., Flannery, B. P., Teukolsky, S. A., \& Vetterling, W. T. 1992, Numerical Recepies in Fortran 77, 2nd edn. (Cambridge: Cambridge Univ. Press)

Ramírez, I., \& Meléndez, J. 2005, AJ, 626, 465

Sanford, R. F. 1937, ApJ, 86, 154

Santos, N. C., Israelian, G., \& Mayor, M. 2004 A\&A, 415, 1153

Schlegel, D. J., Finkbeiner, D. P., \& Davis, M. 1998, ApJ, 500, 525

Simon, K. P., \& Sturm, E., 1994, A\&A, 281, 286

Southworth, J., Maxted, P. F. L., \& Smalley, B., 2004a, MNRAS, 351, 1277

Southworth, J., Zucker, S., Maxted, P. F. L., \& Smalley, B., 2004b, MNRAS, 355,986

Southworth, J., Maxted, P. F. L., \& Smalley, B. 2005, A\&A, 429, 645

Strohmeier, W., Kippenhahn, R., \& Geyer, E. 1956, Kleine Ver. der RemeisSternwarte Bamberg, No. 15

Struve, O. 1945, ApJ, 102, 74

Tomasella, L., Munari, U., Siviero, A., et al. 2007,

[arXiv:astro-ph/07123387]

Torres, G., Neuhäuser, R., \& Guenther, E. W. 2002, AJ, 123, 1701

Torres, G., Lacy, C. H., Marschall, L. A., Sheets, H. A, \& Mader, J. A. 2006, AJ, 640, 1018

Torres, G., Stefanik, R. P., Andersen, J., et al. 1997, AJ, 114, 2764

Valenti, J., \& Piskunov, N. 1996, A\&AS, 118, 595

VandenBerg, D. A., Swenson, F. J., Rogers, F. J., Iglesias, C. A., \& Alexander, D. R. 2000, ApJ, 532, 430

VandenBerg, D. A., Bergbusch, P. A., \& Dowler, P. D. 2006 ApJS, 162, 375

van Hamme, W., 1993, AJ, 106, 2096

Vaz, L. P. R. 1984, Ph.D. Thesis, Copenhagen University (unpublished)

Vaz, L. P. R. 1986, RMA\&A, 12, 177

Vaz, L. P. R., Andersen, J., \& Rebello Soares, M. C. A. 1995, A\&A, 301, 693

Vaz, L. P. R., \& Nordlund, Å. 1985, A\&A, 147, 281

Walker, R. L. 1970, AJ, 75, 720

Wallace, L., Hinkle, K., \& Livingston, W. 1998, An atlas of the spectrum of the solar photosphere from 13500 to $28000 \mathrm{~cm}^{-1}$ (3570 to $7405 \mathrm{~A}$ ), Tucson, AZ: NOAO

Wilson, R. E., 1993, in New Frontiers in Binary Star Research, ed. K.-C. Leung, \& I.-S. Nha, ASP Conf. Ser. 38, 91

Wilson, R. E. 1994, PASP, 106, 921

Wilson, R. E., \& Devinney, E. J. 1971, ApJ, 166, 605

Wood, D. B. 1971, AJ, 76, 701

Wood D. B. 1972, A Computer Program for Modeling Non-Spherical Eclipsing Binary Star Systems, Goddard Space Flight Center, Greenbelt, Maryland

Wood, F. B. 1946, Princeton Contrib., 21, 1

Zhai, D.-S., Zhang, R.-X., \& Zhang, J.-T. 1983, Acta Astrophys. Sinica, 2, 224

Zucker, S., \& Mazeh, T. 1994, ApJ, 420, 806 
J. V. Clausen et al.: Absolute dimensions of eclipsing binaries. XXVI., Online Material $p 1$

\section{Appendix A: Radial velocity tables}

Table A.1. Radial velocities (corrections applied) of AD Boo and residuals from the final spectroscopic orbit.

\begin{tabular}{|c|c|c|c|c|c|}
\hline $\begin{array}{c}\text { HJD } \\
-2400000\end{array}$ & Phase & $\begin{array}{c}R V_{\mathrm{p}} \\
\mathrm{km} \mathrm{s}^{-1}\end{array}$ & $\begin{array}{c}R V_{\mathrm{s}} \\
\mathrm{km} \mathrm{s}^{-1}\end{array}$ & $\begin{array}{l}(\mathrm{O}-\mathrm{C})_{\mathrm{p}} \\
\mathrm{km} \mathrm{s}^{-1}\end{array}$ & $\begin{array}{l}(\mathrm{O}-\mathrm{C})_{\mathrm{S}} \\
\mathrm{km} \mathrm{s}^{-1}\end{array}$ \\
\hline 47555.8293 & 0.3938 & -83.75 & 58.17 & -1.57 & -2.09 \\
\hline 47598.8459 & 0.1868 & -114.44 & 97.36 & -0.05 & -0.57 \\
\hline 47612.6969 & 0.8820 & 56.10 & -103.17 & 0.92 & -2.81 \\
\hline 47613.8580 & 0.4432 & -53.94 & 25.71 & -0.34 & -1.13 \\
\hline 47628.7443 & 0.6388 & 66.83 & -113.43 & 2.09 & -1.89 \\
\hline 47640.6739 & 0.4052 & -77.17 & 57.34 & -1.11 & 4.23 \\
\hline 47642.7577 & 0.4125 & -69.91 & 49.99 & 2.09 & 1.63 \\
\hline 47643.6147 & 0.8267 & 75.39 & -124.58 & -2.14 & 1.91 \\
\hline 47644.7409 & 0.3711 & -93.70 & 76.77 & -0.31 & 3.39 \\
\hline 47675.5822 & 0.2788 & -123.92 & 103.72 & -3.01 & -1.84 \\
\hline 47676.5584 & 0.7507 & 88.38 & -142.11 & -1.23 & -1.48 \\
\hline 47693.7772 & 0.0738 & -66.80 & 36.38 & -2.83 & -2.59 \\
\hline 47700.6648 & 0.4030 & -77.85 & 59.20 & -0.59 & 4.69 \\
\hline 47701.7853 & 0.9446 & 20.07 & -53.80 & 0.42 & 5.02 \\
\hline 47702.6453 & 0.3603 & -97.54 & 79.78 & 0.61 & 0.84 \\
\hline 47702.7425 & 0.4073 & -74.63 & 52.97 & 0.25 & 1.24 \\
\hline 47703.6426 & 0.8424 & 70.23 & -120.29 & -1.99 & 0.00 \\
\hline 47703.7439 & 0.8914 & 51.04 & -97.68 & 0.62 & -2.88 \\
\hline 47729.5844 & 0.3819 & -86.88 & 72.30 & 1.36 & 4.95 \\
\hline 47731.5528 & 0.3334 & -106.61 & 91.39 & 1.81 & 0.44 \\
\hline 47734.5463 & 0.7803 & 88.81 & -141.47 & 1.12 & -3.09 \\
\hline 47957.8075 & 0.6982 & 81.97 & -130.23 & -2.07 & 3.88 \\
\hline 47958.8221 & 0.1886 & -115.20 & 94.94 & -0.34 & -3.53 \\
\hline 47959.8666 & 0.6935 & 81.81 & -127.99 & -1.19 & 4.90 \\
\hline 47960.9249 & 0.2050 & -119.65 & 105.59 & -1.20 & 2.92 \\
\hline 48279.8560 & 0.3669 & -96.11 & 72.02 & -0.82 & -3.58 \\
\hline 48280.9257 & 0.8840 & 53.24 & -104.72 & -0.95 & -5.51 \\
\hline 48311.9322 & 0.8716 & 59.11 & -101.51 & -1.02 & 4.64 \\
\hline 48499.5443 & 0.5577 & 19.69 & -53.87 & -1.43 & 6.67 \\
\hline 48638.8910 & 0.9138 & 36.51 & -79.95 & -1.70 & 0.57 \\
\hline 48639.9724 & 0.4365 & -57.70 & 32.06 & 0.06 & 0.35 \\
\hline 48699.9584 & 0.4319 & -61.66 & 32.15 & -1.12 & -2.80 \\
\hline 48701.8654 & 0.3537 & -101.73 & 83.27 & -0.83 & 1.12 \\
\hline 48704.9191 & 0.8298 & 79.57 & -125.73 & 3.02 & -0.37 \\
\hline 48705.8840 & 0.2962 & -120.74 & 107.80 & -2.53 & 5.40 \\
\hline 48706.8287 & 0.7528 & 92.19 & -139.10 & 2.59 & 1.51 \\
\hline 48711.8041 & 0.1578 & -106.84 & 83.82 & -1.50 & -3.52 \\
\hline 48725.8331 & 0.9390 & 21.13 & -60.51 & -2.04 & 2.42 \\
\hline 48728.8111 & 0.3785 & -93.65 & 70.35 & -3.74 & 1.05 \\
\hline 48730.9634 & 0.4188 & -69.17 & 44.35 & -0.84 & 0.28 \\
\hline 48754.5676 & 0.8284 & 77.25 & -127.14 & 0.26 & -1.27 \\
\hline 48759.7935 & 0.3545 & -99.14 & 87.17 & 1.47 & 5.36 \\
\hline 48783.6614 & 0.8915 & 52.54 & -97.21 & 2.17 & -2.48 \\
\hline 48783.7736 & 0.9457 & 18.44 & -58.86 & -0.54 & -0.83 \\
\hline 48784.7101 & 0.3984 & -78.44 & 62.33 & 1.33 & 4.89 \\
\hline 48810.6101 & 0.9177 & 34.75 & -77.67 & -1.21 & 0.21 \\
\hline 48824.6164 & 0.6879 & 79.52 & -130.74 & -2.12 & 0.57 \\
\hline 48828.5744 & 0.6011 & 44.50 & -88.00 & -1.96 & 2.16 \\
\hline 48840.5419 & 0.3858 & -87.71 & 69.26 & -1.42 & 4.19 \\
\hline 49106.8924 & 0.1318 & -91.82 & 76.57 & 2.87 & 1.68 \\
\hline 49111.6651 & 0.4388 & -57.14 & 30.24 & -0.78 & 0.17 \\
\hline 49112.6802 & 0.9294 & 27.39 & -73.06 & -1.63 & -3.29 \\
\hline 49114.7754 & 0.9422 & 22.66 & -59.87 & 1.46 & 0.75 \\
\hline 49115.7720 & 0.4239 & -65.94 & 41.63 & -0.59 & 1.05 \\
\hline 49117.7719 & 0.3906 & -84.69 & 61.93 & -0.83 & -0.30 \\
\hline 49136.7641 & 0.5709 & 28.22 & -72.23 & -0.97 & -2.26 \\
\hline 49138.8295 & 0.5692 & 24.31 & -67.62 & -3.89 & 1.19 \\
\hline 49140.6382 & 0.4435 & -54.56 & 19.20 & -1.14 & -7.43 \\
\hline 49141.6541 & 0.9345 & 28.08 & -65.19 & 2.17 & 0.94 \\
\hline
\end{tabular}


J. V. Clausen et al.: Absolute dimensions of eclipsing binaries. XXVI., Online Material p 2

Table A.1. Radial velocities (corrections applied) of AD Boo and residuals from the final spectroscopic orbit.

\begin{tabular}{|c|c|c|c|c|c|}
\hline $\begin{array}{c}\text { HJD } \\
-2400000\end{array}$ & Phase & $\begin{array}{c}R V_{\mathrm{p}} \\
\mathrm{km} \mathrm{s}^{-1}\end{array}$ & $\begin{array}{c}R V_{\mathrm{s}} \\
\mathrm{km} \mathrm{s}^{-1}\end{array}$ & $\begin{array}{l}(\mathrm{O}-\mathrm{C})_{\mathrm{p}} \\
\mathrm{km} \mathrm{s}^{-1}\end{array}$ & $\begin{array}{l}(\mathrm{O}-\mathrm{C})_{\mathrm{s}} \\
\mathrm{km} \mathrm{s}^{-1}\end{array}$ \\
\hline 49142.6823 & 0.4315 & -59.39 & 41.82 & 1.38 & 6.59 \\
\hline 49146.7972 & 0.4206 & -66.61 & 40.58 & 0.71 & -2.30 \\
\hline 49173.6118 & 0.3820 & -85.69 & 65.83 & 2.53 & -1.49 \\
\hline 49174.5980 & 0.8587 & 65.86 & -114.47 & 0.03 & -1.66 \\
\hline 49174.7590 & 0.9365 & 25.50 & -66.53 & 0.77 & -1.78 \\
\hline 49175.6609 & 0.3724 & -93.04 & 71.89 & -0.27 & -0.76 \\
\hline 49401.7803 & 0.6718 & 76.53 & -127.27 & -0.54 & -1.31 \\
\hline 49447.7007 & 0.8684 & 63.10 & -116.37 & 1.52 & -8.53 \\
\hline 49564.6149 & 0.3813 & -86.88 & 69.00 & 1.68 & 1.28 \\
\hline 49761.8372 & 0.7127 & 85.16 & -131.47 & -1.55 & 5.76 \\
\hline 49765.8501 & 0.6524 & 69.42 & -116.29 & -0.85 & 1.72 \\
\hline 49767.8090 & 0.5993 & 45.62 & -89.81 & 0.15 & -0.81 \\
\hline 49825.8712 & 0.6648 & & -122.47 & -0.65 & 0.80 \\
\hline & 0.3415 & 104 & & 0.85 & -0.66 \\
\hline 498 & 0.8052 & 8 & 125 & -0.21 & 7.60 \\
\hline 498 & 0.4464 & -52.53 & 26.95 & -0.92 & 2.43 \\
\hline 49901 & 0.2673 & -123.33 & 105.21 & -1.30 & -1.65 \\
\hline 49909 & 1330 & -96 & 77.05 & -0.94 & 1.51 \\
\hline 49920.5892 & 0.4487 & -48.66 & 24.62 & 1.49 & 1.81 \\
\hline 49939.5702 & 0.6235 & 60.30 & -99.67 & 2.46 & 3.80 \\
\hline 49965.5149 & 0.1644 & 105.60 & 96.18 & 2.08 & 6.10 \\
\hline 49979.5013 & 0.9250 & 36. & -70 & 4.43 & 2.43 \\
\hline 50119.8669 & 0.7736 & 87. & -134 & -0.62 & 4.82 \\
\hline 50126.9140 & 0.1800 & -113.41 & 96.07 & -0.86 & 0.30 \\
\hline 50140.7929 & 0.8886 & 51.90 & -99.18 & 0.06 & -2.73 \\
\hline 5014 & 0.8320 & 77.74 & -125.27 & 1.91 & -0.76 \\
\hline & 45 & & 127 & -1.71 & -0.48 \\
\hline & 16 & -73 & & -0.57 & 5.10 \\
\hline & 7 & 113 & & 0.98 & -2.37 \\
\hline & 9 & & 130 & -0.70 & 0.37 \\
\hline & 06 & & 131 & 3.60 & 0.97 \\
\hline 505 & 0.3565 & 100 & 76 & -0.45 & -4.51 \\
\hline 50535.6409 & 0.7464 & 89.82 & 139.25 & 0.23 & 1.35 \\
\hline 50535.6560 & 0.7537 & 91.99 & -146.77 & 2.40 & -6.18 \\
\hline 50535.6710 & 0.7610 & 90.27 & -135 & 0.91 & 4.52 \\
\hline 50540.8206 & 0.2502 & 124. & 109 & -1.43 & 1.41 \\
\hline 50541.7939 & 0.7206 & 86.82 & -140.28 & -0.99 & -1.76 \\
\hline 50543.8365 & 0.7080 & 83.48 & -131.91 & -2.45 & 4.41 \\
\hline 50561.7089 & 0.3469 & 105.72 & 80.35 & -2.15 & -4.92 \\
\hline 50563.7115 & 0.3149 & -113.48 & & 0.46 & -0.96 \\
\hline 50564.6929 & 0.7893 & & 139.03 & -0.04 & -2.17 \\
\hline & 0.2523 & 20.05 & & 2.59 & -6.47 \\
\hline & & 173 & 1 & -0.42 & -2.68 \\
\hline & & 9 & 1 & .38 & 0.90 \\
\hline & 3 & 0 & 1 & 1.04 & 1.60 \\
\hline & 0 & 5 & & -0.09 & 0.69 \\
\hline & 6 & -116 & 98 & -2.72 & 1.63 \\
\hline 50589.5959 & 0.8267 & 77.20 & -127.53 & -0.33 & -1.03 \\
\hline 50590.6390 & 0.3309 & 107.69 & 93.36 & 1.55 & 1.46 \\
\hline 50591.6685 & 0.8285 & 75.92 & -124.18 & -1.04 & 1.65 \\
\hline 50592.6487 & 0.3023 & -116.76 & 100.03 & 0.21 & -0.91 \\
\hline 50593.5959 & 0.7602 & 87.43 & -140.95 & -1.97 & -0.57 \\
\hline 50595.6617 & 0.7587 & 86.84 & -135.87 & -2.62 & 4.57 \\
\hline 50596.5967 & 0.2107 & -117.38 & 102.96 & 2.05 & -0.86 \\
\hline 50597.6074 & 0.6992 & 82.68 & -135.60 & -1.58 & -1.24 \\
\hline 50619.6064 & 0.3329 & -106.61 & 94.13 & 1.98 & 2.99 \\
\hline 50619.6215 & 0.3402 & -106.01 & 91.36 & 0.06 & 3.16 \\
\hline & & & 126.68 & 0.33 & 1.28 \\
\hline 50621.5810 & 0.2873 & 120.35 & 107.51 & -0.60 & 3.32 \\
\hline 50622.6012 & 0.7805 & 89.09 & -133.20 & 1.41 & 5.16 \\
\hline 50623.6005 & 0.2635 & -122.58 & 108.27 & -0.31 & 1.13 \\
\hline 50624.6042 & 0.7487 & 87.14 & -139.68 & -2.47 & 0.94 \\
\hline & 0.2367 & -122.61 & 108.53 & -0.33 & 1.37 \\
\hline & 02204 & -123.42 & 109.14 & -2.59 & 3.68 \\
\hline 50629.5948 & 0.1610 & -106.47 & 90.60 & 0.01 & 1.92 \\
\hline
\end{tabular}


J. V. Clausen et al.: Absolute dimensions of eclipsing binaries. XXVI., Online Material p 3

Table A.2. Radial velocities (corrections applied) of VZ Hya and residuals from the final spectroscopic orbit.

\begin{tabular}{crrrrr}
\hline \hline $\begin{array}{c}\text { HJD } \\
-2400000\end{array}$ & Phase & \multicolumn{1}{c}{$R V_{\mathrm{p}}$} & \multicolumn{1}{c}{$R V_{\mathrm{s}}$} & \multicolumn{1}{c}{$(\mathrm{O}-\mathrm{C})_{\mathrm{p}}$} & \multicolumn{1}{c}{$(\mathrm{O}-\mathrm{C})_{\mathrm{s}}$} \\
\hline 47555.7226 & 0.8107 & 82.13 & -101.37 & -1.40 & 0.94 \\
47558.7158 & 0.8413 & 75.81 & -89.61 & 0.66 & 3.40 \\
47568.6242 & 0.2530 & -99.07 & 101.23 & 0.40 & 0.51 \\
47570.7110 & 0.9715 & 13.91 & -20.42 & 1.56 & 2.92 \\
47574.6105 & 0.3141 & -93.26 & 92.10 & -1.38 & -0.20 \\
47575.6910 & 0.6862 & 82.32 & -100.78 & -0.50 & 0.74 \\
47577.6268 & 0.3527 & -79.70 & 81.21 & 0.70 & 1.64 \\
47583.6110 & 0.4132 & -54.33 & 49.75 & -0.51 & -0.33 \\
47587.6242 & 0.7950 & 86.63 & -105.73 & 0.05 & -0.03 \\
47603.5188 & 0.2678 & -98.83 & 100.09 & 0.06 & 0.00 \\
47609.5595 & 0.3477 & -80.89 & 83.10 & 1.27 & 1.58 \\
47613.5894 & 0.7352 & 90.37 & -110.66 & 0.43 & -1.24 \\
47628.5692 & 0.8930 & 54.76 & -71.57 & 0.23 & -1.44 \\
47642.5540 & 0.7082 & 85.75 & -106.46 & -1.35 & -0.19 \\
47645.5312 & 0.7333 & 89.68 & -108.31 & -0.15 & 0.99 \\
47664.5534 & 0.2830 & -97.70 & 100.29 & -0.25 & 1.81 \\
47670.6171 & 0.3708 & -74.23 & 70.09 & -0.82 & -1.72 \\
47671.6217 & 0.7167 & 87.91 & -106.94 & -0.37 & 0.64 \\
47672.6262 & 0.0626 & -39.66 & 38.82 & 1.29 & 3.02 \\
47899.7202 & 0.2549 & -101.14 & 100.79 & -1.70 & 0.10 \\
47905.7028 & 0.3149 & -92.07 & 93.39 & -0.36 & 1.27 \\
47928.7245 & 0.2416 & -98.52 & 102.25 & 0.83 & 1.66 \\
47941.6387 & 0.6882 & 83.84 & -100.78 & 0.56 & 1.25 \\
47956.6250 & 0.8482 & 72.18 & -91.00 & -0.66 & -0.55 \\
47957.6126 & 0.1883 & -91.30 & 96.46 & 1.14 & 3.54 \\
47959.5793 & 0.8654 & 65.89 & -83.00 & -0.56 & 0.36 \\
47960.5813 & 0.2105 & -95.81 & 98.29 & 0.76 & 0.78 \\
48191.8651 & 0.8454 & 74.21 & -89.36 & 0.41 & 2.16 \\
48191.8800 & 0.8505 & 72.28 & -87.06 & 0.25 & 2.50 \\
48226.7915 & 0.8712 & 64.13 & -83.69 & -0.02 & -2.88 \\
48230.9468 & 0.3019 & -96.99 & 89.83 & -2.51 & -5.36 \\
48236.8621 & 0.3386 & -87.41 & 83.89 & -2.27 & -0.94 \\
48252.8201 & 0.8332 & 77.88 & -94.81 & 0.22 & 0.99 \\
48256.8706 & 0.2279 & -98.67 & 99.73 & -0.10 & 0.00 \\
48258.8791 & 0.9195 & 42.00 & -56.01 & 0.56 & -0.40 \\
48259.7319 & 0.2131 & -96.77 & 97.61 & 0.17 & -0.31 \\
48264.7826 & 0.9521 & 22.39 & -34.46 & -1.16 & 1.30 \\
48280.7728 & 0.4578 & -30.24 & 23.96 & -0.82 & 0.95 \\
48281.7752 & 0.8030 & 82.86 & -103.45 & -2.28 & 0.64 \\
48290.6911 & 0.8729 & 63.96 & -84.32 & 0.52 & -4.30 \\
48315.6410 & 0.4636 & -24.29 & 18.58 & 1.82 & -0.76 \\
48354.5562 & 0.8627 & 68.11 & -84.77 & 0.59 & -0.22 \\
\hline & & & & &
\end{tabular}


J. V. Clausen et al.: Absolute dimensions of eclipsing binaries. XXVI., Online Material p 4

Table A.3. Radial velocities (corrections applied) of WZ Oph and residuals from the final spectroscopic orbit.

\begin{tabular}{crrrrr}
\hline \hline $\begin{array}{c}\text { HJD } \\
-2400000\end{array}$ & Phase & \multicolumn{1}{r}{$R V_{\mathrm{p}}$} & \multicolumn{1}{c}{$R V_{\mathrm{s}}$} & $\begin{array}{r}(\mathrm{O}-\mathrm{C})_{\mathrm{p}} \\
\mathrm{km} \mathrm{s}^{-1}\end{array}$ & \multicolumn{1}{c}{$(\mathrm{O}-\mathrm{C})_{\mathrm{s}}$} \\
$\mathrm{km} \mathrm{s}^{-1}$ & $\mathrm{~km} \mathrm{~s}^{-1}$ \\
\hline 47583.9253 & 0.4058 & -78.81 & 22.61 & -0.94 & 1.19 \\
47605.8830 & 0.6545 & 45.26 & -101.95 & 0.37 & 0.06 \\
47608.8786 & 0.3705 & -94.35 & 35.97 & -1.48 & -0.54 \\
47612.8755 & 0.3259 & -107.37 & 49.40 & -0.15 & -1.54 \\
47628.8294 & 0.1394 & -95.42 & 41.35 & 1.13 & 1.14 \\
47639.8265 & 0.7681 & 58.80 & -117.67 & -1.03 & -0.63 \\
47641.8367 & 0.2486 & -116.31 & 59.25 & 0.81 & -1.64 \\
47643.7878 & 0.7150 & 60.26 & -113.94 & 1.99 & 1.53 \\
47644.8166 & 0.9609 & -5.58 & -47.03 & 1.20 & 3.03 \\
47645.8002 & 0.1960 & -111.58 & 55.62 & 0.49 & -0.20 \\
47662.6849 & 0.2320 & -117.86 & 60.36 & -1.30 & 0.03 \\
47664.7592 & 0.7279 & 58.48 & -117.41 & -1.07 & -0.66 \\
47674.8228 & 0.1334 & -93.47 & 39.12 & 0.89 & 1.12 \\
47675.7352 & 0.3515 & -99.81 & 43.29 & -0.14 & -0.06 \\
47689.7883 & 0.7107 & 56.82 & -117.76 & -0.89 & -2.85 \\
47691.7534 & 0.1804 & -110.29 & 53.39 & -1.52 & 0.89 \\
47691.9235 & 0.2211 & -116.21 & 59.69 & -0.55 & 0.26 \\
47693.8686 & 0.6860 & 54.15 & -110.29 & 0.82 & 0.21 \\
47693.9378 & 0.7025 & 56.23 & -112.40 & -0.26 & 1.28 \\
47700.6733 & 0.3126 & -109.29 & 55.41 & 1.06 & 1.32 \\
47723.6572 & 0.8065 & 56.53 & -111.37 & 1.66 & 0.68 \\
47725.6208 & 0.2759 & -117.06 & 61.70 & -1.10 & 1.98 \\
47727.6324 & 0.7567 & 58.66 & -118.05 & -1.67 & -0.51 \\
47729.6085 & 0.2291 & -116.36 & 60.66 & 0.00 & 0.53 \\
47731.6271 & 0.7116 & 57.97 & -113.55 & 0.14 & 1.48 \\
47775.5008 & 0.1989 & -112.54 & 57.69 & 0.04 & 1.36 \\
47777.5180 & 0.6811 & 51.47 & -110.36 & -0.74 & -0.99 \\
47779.5228 & 0.1603 & -103.69 & 47.89 & -0.30 & 0.81 \\
47788.5003 & 0.3062 & -111.36 & 56.91 & 0.29 & 1.52 \\
48194.4589 & 0.3441 & -99.98 & 43.14 & 2.09 & -2.62 \\
48405.7132 & 0.8410 & 46.85 & -104.96 & 0.56 & -1.54 \\
48409.7815 & 0.8135 & 54.04 & -111.28 & 0.60 & -0.67 \\
48429.7546 & 0.5877 & 17.51 & -73.70 & -0.61 & 1.40 \\
48432.7522 & 0.3042 & -113.49 & 54.28 & -1.47 & -1.48 \\
48462.5915 & 0.4369 & -63.20 & 5.35 & -0.54 & -0.78 \\
48466.5983 & 0.3946 & -81.02 & 25.90 & 1.92 & -0.62 \\
48485.5754 & 0.9308 & 8.77 & -65.98 & -0.27 & -0.01 \\
48491.5446 & 0.3576 & -98.40 & 39.30 & -0.81 & -1.96 \\
48499.5903 & 0.2808 & -114.65 & 57.64 & 0.82 & -1.59 \\
48501.5840 & 0.7574 & 59.46 & -117.06 & -0.85 & 0.46 \\
\hline & & & & & \\
\hline
\end{tabular}

